\title{
The Topological Vertex
}

\section{Citation}

Aganagic, Mina, Albrecht Klemm, Marcos Mari\#o, and Cumrun Vafa. 2004. "The Topological Vertex." Communications in Mathematical Physics 254 (2): 425-78. https://doi.org/10.1007/ s00220-004-1162-z.

\section{Permanent link}

http://nrs.harvard.edu/urn-3:HUL.InstRepos:41385008

\section{Terms of Use}

This article was downloaded from Harvard University's DASH repository, and is made available under the terms and conditions applicable to Other Posted Material, as set forth at http:// nrs.harvard.edu/urn-3:HUL.InstRepos:dash.current.terms-of-use\#LAA

\section{Share Your Story}

The Harvard community has made this article openly available.

Please share how this access benefits you. Submit a story.

\section{Accessibility}


hep-th/0305132

CALT-68-2439

HUTP-03/A032

HU-EP-03/24

CERN-TH/2003-111

\title{
The Topological Vertex
}

\author{
Mina Aganagic, ${ }^{a}$ Albrecht Klemm, ${ }^{b}$ Marcos Mariño, ${ }^{c}$ and Cumrun Vafa ${ }^{a, d}$ \\ a Jefferson Physical Laboratory, Harvard University \\ Cambridge, MA 02138, USA \\ ${ }^{b}$ Humboldt-Universität zu Berlin, Institut für Physik \\ D-10115 Berlin, Germany \\ c Theory Division, CERN, Geneva 23, CH-1211 Switzerland \\ ${ }^{d}$ California Institute of Technology, 452-48, Pasadena, CA 91125, USA
}

\begin{abstract}
We construct a cubic field theory which provides all genus amplitudes of the topological A-model for all non-compact toric Calabi-Yau threefolds. The topology of a given Feynman diagram encodes the topology of a fixed Calabi-Yau, with Schwinger parameters playing the role of Kähler classes of the threefold. We interpret this result as an operatorial computation of the amplitudes in the B-model mirror which is the quantum KodairaSpencer theory. The only degree of freedom of this theory is an unconventional chiral scalar on a Riemann surface. In this setup we identify the B-branes on the mirror Riemann surface as fermions related to the chiral boson by bosonization.
\end{abstract}




\section{Introduction}

Topological strings have been a focus of much interest since they were proposed more than a decade ago [1]. A central question has been how to compute the corresponding amplitudes. There have been two natural approaches available: i) using mirror symmetry to transform the problem to an easier one; ii) mathematical idea of localization. Both approaches can in principle yield answers to all genus amplitudes (at least in the noncompact case). However the computations get more and more involved as one goes to higher genera and neither method becomes very practical.

Ever since the discovery of large $N$ Chern-Simons/topological string duality [2] another approach has opened up: Chern-Simons amplitudes seem to give an efficient way to sum up all genus amplitudes. This idea was developed recently [3, [4] where it was shown that one can compute all genus A-model amplitudes on local toric 3-folds from its relation to Chern-Simons amplitudes. However in trying to obtain amplitudes in this way, one had often to take certain limits. The main aim of the present paper is to bring this line of thought to a natural conclusion by giving the direct answer for the topological string amplitudes, without any need to take any limits.

Toric 3 -folds are characterized by a graph which encodes where the cycles of a $T^{2}$ fibration degenerates. The vertices of this graph are generically trivalent. The computations in [3] and [4] were more natural in the context of tetravalent vertices of the toric graph. To obtain the generic situation of trivalent graph, one had to take particular limits in the Calabi-Yau moduli space. Thus the basic goal is to directly capture the structure of the trivalent vertex. That there should be such a vertex has already been noted [5:6] In this paper we show how this can be achieved. The idea can be summarized, roughly, as putting many brane/anti-brane pairs which effectively chop off the Calabi-Yau to patches with trivial topology of $\mathbf{C}^{3}$. Computing open topological string on $\mathbf{C}^{3}$ defines the cubic topological vertex. Gluing these together yields the closed topological string results (with or without additional D-branes). Thus the full amplitude can be obtained from a cubic field theory, where each Calabi-Yau corresponds to a Feynman graph with some fixed Schwinger times (determined by the Kähler class of the Calabi-Yau).

1 This had also been noted in our discussions with D.-E. Diaconescu and A. Grassi. In particular, progress towards formulation of the vertex in terms of mathematical localization techniques has been made [7]. 
This result can best be understood in the mirror picture as computation of the quantum Kodaira-Spencer theory [8]. The Kodaira-Spencer theory is, in this context of noncompact Calabi-Yau, captured locally by a chiral boson on a Riemann surface. The degrees of freedom on the brane get mapped, in this setup, to coherent states of the chiral boson, and the trivalent vertex gets identified with the quantum correlations of the chiral boson. Moreover, the brane in the B-model gets identified with the fermions of this chiral boson. Thus the fact that knowing amplitudes involving branes leads to closed string results translates to the statement that knowing amplitudes involving fermions leads via bosonization to the full answer for the chiral boson. The topological vertex gets mapped, in this setup, to a state in the three-fold tensor product of the Fock space of a single bosonic string oscillator. To leading order in string coupling and oscillator numbers this is a squeezed state as in the conventional approaches to the operator formulation in the Riemann surface. However the full topological vertex is far more complicated; the chiral scalar is not a conventional field. The full vertex involves infinitely many oscillator terms together with highly non-trivial $g_{s}$ dependence. Nevertheless, we find the following closed formula for this highly non-trivial vertex $|C\rangle$ :

$$
\left\langle t_{n}^{1}, t_{m}^{2}, t_{p}^{3} \mid C\right\rangle=\sum_{Q_{1}, Q_{3}} N_{Q_{1} Q_{3}^{t}}^{R_{1} R_{3}^{t}} q^{\kappa_{R_{2}} / 2+\kappa_{R_{3}} / 2} \frac{W_{R_{2}^{t} Q_{1}} W_{R_{2} Q_{3}^{t}}}{W_{R_{2} 0}} \operatorname{tr}_{R_{1}} V_{1} \operatorname{tr}_{R_{2}} V_{2} \operatorname{tr}_{R_{3}} V_{3} .
$$

where

$$
N_{Q_{1} Q_{3}^{t}}^{R_{1} R_{3}^{t}}=\sum_{R} N_{Q_{1} R}^{R_{1}} N_{Q_{3}^{t} R}^{R_{3}^{t}}
$$

Here $R_{i}, Q_{i}$ are representation of $U(N), N_{R_{i} R_{j}}^{R_{k}}$ is the number of times the representation $R_{k}$ appears in the tensor product of representations $R_{i}$ and $R_{j}, R^{t}$ denotes the representation whose Young Tableau is the transpose of that of $R$ and $W_{R Q}=S_{R \bar{Q}} / S_{00}$ where $S$ is the S-matrix of the modular transformation of the characters of $U(N)_{k}$ WZW for fixed $k+N=2 \pi i / g_{s}$ and $N \rightarrow \infty$. The $t_{n}^{i}$ are the coherent states of a single bosonic string oscillator and they are related to $V_{i}$ by $t_{n}^{i}=\operatorname{tr}\left(V_{i}\right)^{n}$ in the fundamental representation, $\kappa_{R}$ is related to the quadratic Casimir of the representation $R$ and $q=\exp \left(g_{s}\right)$. This is obtained by considering certain amplitudes in the context of large $N$ topological duality [2].

The organization of this paper is as follows: In section 2 we review the relevant facts about local toric Calabi-Yau threefolds including their $T^{2}$ fibration structure and its relation to $(p, q)$ 5-branes. We also review mirror symmetry of these manifolds, where 
mirror geometry reduces, in appropriate sense, to a Riemann surface. In section 3 we discuss how the knowledge of A-model open topological string amplitudes on $\mathbf{C}^{3}$ with 3 sets of Lagrangian D-branes (defining a trivalent vertex) can be used to compute the Amodel amplitudes for all toric Calabi-Yau threefolds with or without D-branes. In section 4 we formulate the vertex in terms of a chiral bosonic oscillator in $1+1$ dimension. In section 5 we formulate the mirror B-model and discuss the interpretation of the vertex in this setup. In section 6 we derive the complete expression for the cubic vertex using the large $N$ topological duality in terms of certain Chern-Simons amplitudes. In section 7 we explain how the vertex can be evaluated explicitly. In section 8 we evaluate the vertex for low excitations and show that it passes some highly non-trivial tests. In section 9 we apply our formalism to a number of examples.

\section{Toric Geometry and Mirror Symmetry}

A smooth Calabi-Yau three-fold can be obtained by gluing together $\mathbf{C}^{3}$ patches in a way that is consistent with Ricci-flatness. For toric Calabi-Yau threefolds the gluing data and the resulting manifold are simple to describe.

The toric Calabi-Yau 3-folds are special Lagrangian $T^{2} \times \mathbf{R}$ fibrations over the base $\mathbf{R}^{3}$ (they are also Lagrangian $T^{3}$ fibrations, but this will not be relevant for us). The geometry of the manifold is encoded in the one dimensional planar graph $\Gamma$ in the base that corresponds to the degeneration locus of the fibration. The edges of the graph are oriented straight lines labeled by vectors $(p, q) \in \mathbf{Z}^{2}$, where the label corresponds to the generator of $H_{1}\left(T^{2}\right)$ which is the shrinking cycle. Changing the orientation on each edge replaces $(p, q) \rightarrow(-p,-q)$ which does not change the Calabi-Yau geometry. The condition of being a Calabi-Yau is equivalent to the condition that on each vertex, if we choose the edges to be incoming with charges $v_{i}=\left(p_{i}, q_{i}\right)$, one must have

$$
\sum_{i} v_{i}=0
$$

If the local geometry of the threefold near the vertex is $\mathbf{C}^{3}$, then the vertex is trivalent. Moreover, for any pair of incoming edges one has that

$$
\left|v_{i} \wedge v_{j}\right|=1
$$

where $\wedge$ denotes the symplectic product on $H^{1}\left(T^{2}\right)$. This condition ensures smoothness. 


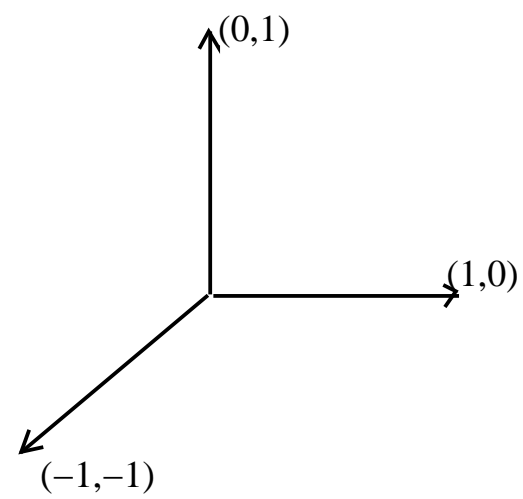

Fig. 1 The degenerate locus of the $T^{2} \times \mathbf{R}$ fibration of $\mathbf{C}^{3}$ in the base $\mathbf{R}^{\mathbf{3}}=$ $\left(r_{\alpha}, r_{\beta}, r_{\gamma}\right)$. This locus is a graph $\Gamma$. The labels $\left(-p_{i}, q_{i}\right)$ correspond to the cycles of $T^{2}$ which vanish over the corresponding edge.

The graph corresponding to $\mathbf{C}^{\mathbf{3}}$ can be obtained as follows. Let $z_{i}$ be complex coordinates on $\mathbf{C}^{3}, i=1,2,3$. The base of the $T^{2} \times \mathbf{R}$ fibration is the image of moment maps $r_{\alpha}(z)=\left|z_{1}\right|^{2}-\left|z_{3}\right|^{2}, r_{\beta}(z)=\left|z_{2}\right|^{2}-\left|z_{3}\right|^{2}$, and $r_{\gamma}(z)=\operatorname{Im}\left(z_{1} z_{2} z_{3}\right)$. The special Lagrangian fibers are then generated by the action of the three "Hamiltonians" $r_{\alpha, \beta, \gamma}$ on $\mathbf{C}^{3}$ via the standard symplectic form $\omega=i \sum_{i} d z_{i} \wedge d \bar{z}_{i}$ on $\mathbf{C}^{3}$ and Poisson brackets, $\partial_{\epsilon} z_{i}=\left\{\epsilon \cdot r, z_{i}\right\}_{\omega}$. In particular, the $T^{2}$ fiber is generated by circle actions

$$
\exp \left(i \alpha r_{\alpha}+i \beta r_{\beta}\right):\left(z_{1}, z_{2}, z_{3}\right) \rightarrow\left(e^{i \alpha} z_{1}, e^{i \beta} z_{2}, e^{-i(\alpha+\beta)} z_{3}\right),
$$

and $r_{\gamma}$ generates the real line $\mathbf{R}$. We will call the cycle generated by $r_{\alpha}$ the $(0,1)$ cycle, the $(1,0)$ cycle is generated by $r_{\beta}$.

We have that the $(0,1)$ cycle degenerates over $z_{1}=0=z_{3}$. This subspace of $\mathbf{C}^{\mathbf{3}}$ projects to the $r_{\alpha}$ and $r_{\gamma}$ vanishing in the base and $r_{\beta} \geq 0$, by their definition. Similarly over $z_{2}=0=z_{3}$, where $(1,0)$-cycle degenerates, $r_{\beta}$ and $r_{\gamma}$ vanish and $r_{\alpha} \geq 0$, and 1cycle parameterized by $\alpha+\beta$ degenerates over $z_{1}=0=z_{2}$ where $r_{\alpha}-r_{\beta}=0=r_{\gamma}$ and $r_{\alpha} \leq 0$ degenerate. To correlate the cycles unambiguously with the lines in the graph (up to $(q, p) \rightarrow(-q,-p)$ ) we will let a $(-q, p)$ cycle of the $T^{2}$ degenerate over an edge that corresponds to $p r_{\alpha}+q r_{\beta}=0$. The places in the base where $T^{2}$ fibers degenerate are correlated with the zero's of the corresponding Hamiltonians. This yields the graph in fig. 1 (drawn in the $r_{\gamma}=0$ plane).

Above we have made a choice for generators of $H_{1}\left(T^{2}\right)$ to be the 1-cycles generated by $r_{\alpha}$ and $r_{\beta}$. Other choices will differ from this one by an $S L(2, \mathbf{Z})$ transformation that acts on the $T^{2}$. We can have $r_{\alpha}$ generate a $(p, q) 1$-cycle and $r_{\beta}$ the $(t, s) 1$-cycle where 
$p s-q t=1$. This of course is a symmetry of $\mathbf{C}^{3}$. However, when gluing different $\mathbf{C}^{3}$ 's together, as we will discuss below, the relative choices will matter and will give rise to different geometries.

\subsection{More general geometries}

Other toric Calabi-Yau threefolds can be obtained by gluing together $\mathbf{C}^{3}$ 's. First, one adds more coordinates e.g. $z_{4}, \ldots, z_{N+3}$, so that flat patches are described by certain triples of the coordinates. Gluing different patches corresponds, in terms of the base, to identifying some of the coordinates by $N$ linear relations:

$$
\sum_{i} Q_{i}^{A}\left|z_{i}\right|^{2}=t^{A}
$$

where $Q_{A}, A=1, \ldots N$ are integral charges satisfying

$$
\sum_{i} Q_{i}^{A}=0
$$

which is the Calabi-Yau constraint. Finally, one divides the space of solutions to (2.5) by $U(1)^{N}$ action on $z^{\prime} s$ where the $A$-th $U(1)$ acts on $z_{i}$ by

$$
z_{i} \rightarrow \exp \left(i Q_{i}^{A} \theta_{A}\right) z_{i}
$$

The $N$ parameters $t^{A}$ are Kähler moduli of the Calabi-Yau. The mathematical construction above arises in the physical context of two-dimensional linear sigma model with $\mathcal{N}=(2,2)$ supersymmetry on the Higgs branch [9]. The theory has $N+3$ chiral fields, whose lowest components are $z$ 's, which are charged under $N$ vector multiplets with charges $Q_{i}^{A}$. The equations (2.4) give minima of the D-term potential as solutions. Dividing by the $U(1)^{N}$ gauge group, the Higgs branch is a Kähler manifold, and when (2.5) holds, the theory flows to a two dimensional conformal sigma model in the IR.

From the linear sigma model data described above, i.e. the set of $N+3$ coordinates $z_{i}$ 's and the D-term equations one can construct the graph $\Gamma$ corresponding to the toric CalabiYau manifold. First, we must find a decomposition of the set of all coordinates $\left\{z_{i}\right\}_{i=1}^{N+3}$ into triplets $U_{\alpha}=\left(z_{i_{a}}, z_{j_{a}}, z_{k_{a}}\right)$ that correspond to the decomposition of $X$ into $\mathbf{C}^{\mathbf{3}}$ patches. We will describe this below in an example, but it should be clear how to do this in general. We can pick one of the $\mathbf{C}^{3}$ patches, say $U_{1}$ and in this patch we get the Hamiltonians $r_{\alpha}=\left|z_{i_{1}}\right|^{2}-\left|z_{i_{3}}\right|^{2}, r_{\beta}=\left|z_{i_{2}}\right|^{2}-\left|z_{i_{3}}\right|^{2}$ which generate the $T^{2}$ fiber in this patch. As it 
turns out, these can serve as global coordinates in the base $\mathbf{R}^{\mathbf{3}}$. Correspondingly, they generate a globally defined $T^{2}$ fiber 2 . We can call the cycle generated by $r_{\alpha}$ the $(1,0)$ cycle, and that generated by $r_{\beta}$ the $(0,1)$ cycle. The $(2.4)$ equation then can be used to find the action of $r_{\alpha, \beta}$ on the other patches. Namely, in the $U_{a \neq 1}$ patch, we can solve for all the other $z$ 's in terms of $z_{i_{a}}, \ldots, z_{k_{a}}$ using (2.4), since this is by the definition what we mean by the $U_{a}$ patch. The degenerate locus in this patch is then found analogously to the case of the $\mathbf{C}^{\mathbf{3}}$ above, where we use the $r_{\alpha}$ and $r_{\beta}$ as generators of the fiber globally.

Example $: \mathcal{O}(-3) \rightarrow \mathbf{P}^{2}$ A familiar example of a Calabi-Yau manifold, $X$, of this type is the $\mathcal{O}(-3)$ bundle over $\mathbf{P}^{2}$. In this case, there are four coordinates $z_{0}, z_{1}, z_{2}, z_{3}$, and the D-term constraint is

$$
\left|z_{1}\right|^{2}+\left|z_{2}\right|^{2}+\left|z_{3}\right|^{2}-3\left|z_{0}\right|^{2}=t
$$

There are three patches $U_{i}$ defined by $z_{i} \neq 0$, for $i=1,2,3$, since at least one of these three coordinates must be non-zero in $X$. All of these three patches look like $\mathbf{C}^{3}$. For example, for $z_{3} \neq 0$, we can "solve" for $z_{3}$ in terms of the other three unconstrained coordinates which then parameterize $\mathbf{C}^{\mathbf{3}}: U_{3}=\left(z_{0}, z_{1}, z_{2}\right)$. Namely, in this patch, we can use (2.6) to solve for the absolute value of $z_{3}$, in terms of $z_{0,1,2}$, and moreover its phase can be gauged away by dividing with the $U(1)$ action of the symplectic quotient construction: $\left(z_{0}, z_{1}, z_{2}, z_{3}\right) \rightarrow\left(e^{-3 i \theta} z_{0}, e^{i \theta} z_{1}, e^{i \theta} z_{2}, e^{i \theta} z_{3}\right)$. We are left with the space of three unconstrained coordinates $z_{0}, z_{1}, z_{2}$ as we claimed and this is of course $\mathbf{C}^{3}$. Similar statement holds for the other two patches.

Now let us construct the corresponding degeneration graph $\Gamma$. Let the $T^{2}$ fiber in the $U_{3}=\left(z_{0}, z_{1}, z_{2}\right)$ patch be generated by $r_{\alpha}$ and $r_{\beta}$ where $r_{\alpha}=\left|z_{1}\right|^{2}-\left|z_{0}\right|^{2}$ and $r_{\beta}=$ $\left|z_{2}\right|^{2}-\left|z_{0}\right|^{2}$. The graph of the degenerate fibers in the $r_{\alpha}-r_{\beta}$ plane is the same as in our first $\mathbf{C}^{3}$ example, fig. 1 (the third direction in the base, $r_{\gamma}$ is now given by the gauge invariant product $\left.r_{\gamma}=\operatorname{Im}\left(z_{0} z_{1} z_{2} z_{3}\right)\right)$. The same two Hamiltonians $r_{\alpha, \beta}$ generate the action in the $U_{2}=\left(z_{0}, z_{1}, z_{3}\right)$ patch, where we use the (2.6) constraint to rewrite them as follows. Since both $z_{0}$ and $z_{1}$ are the coordinates of this patch $r_{\alpha}$ does not change, $r_{\alpha}=\left|z_{1}\right|^{2}-\left|z_{0}\right|^{2}$. On the other hand, $r_{\beta}$ changes as $z_{2}$ is not a natural coordinate here, so instead we have $r_{\beta}=t+2\left|z_{0}\right|^{2}-\left|z_{1}\right|^{2}-\left|z_{3}\right|^{2}$, and hence

$$
\exp \left(i \alpha r_{\alpha}+i \beta r_{\beta}\right):\left(z_{0}, z_{1}, z_{3}\right) \rightarrow\left(e^{i(-\alpha+2 \beta)} z_{0}, e^{i(\alpha-\beta)} z_{1}, e^{-i \beta} z_{3}\right)
$$

2 The third coordinate in the base is $r_{\gamma}=\operatorname{Im}\left(\prod_{i=1}^{N+3} z_{i}\right)$ which is manifestly gauge invariant and moreover, patch by patch, can be identified with the coordinate used in the $\mathbf{C}^{\mathbf{3}}$ example above. 


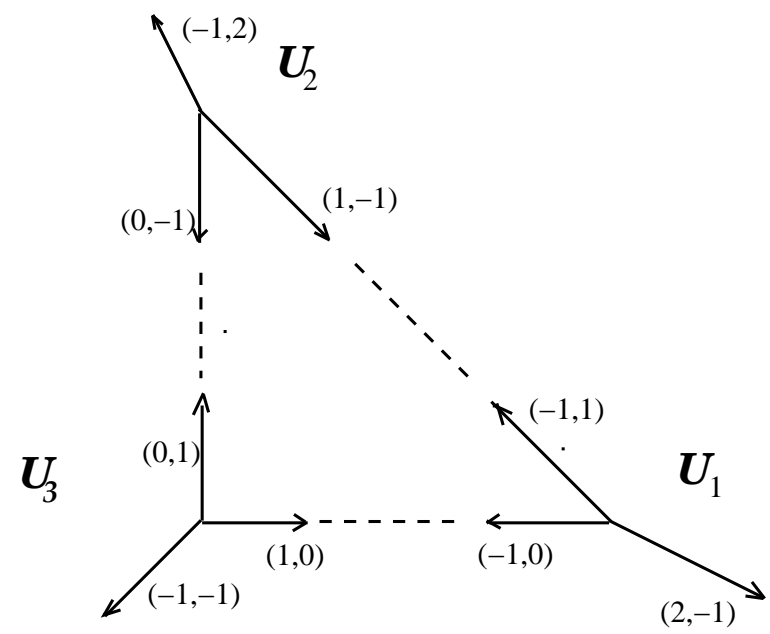

Fig. 2 The graph of $\mathcal{O}(-3) \rightarrow \mathbf{P}^{\mathbf{2}}$. The manifold is built out of three $\mathbf{C}^{3}$ patches with the different orientations as in the figure. The transition functions correspond to $S L(2, \mathbf{Z})$ transformations of the $T^{2}$ fibers as one goes from one patch to the next.

We see from the above that the fibers degenerate over three lines: i) $r_{\alpha}+r_{\beta}=0$, and since $z_{0}=0=z_{3}$ there, $t \geq r_{\alpha} \geq 0$ where the fact that we have to stop when $r_{\alpha}=t$ comes from (2.6). Over this line $(-1,1)$ cycle degenerates. ii) There is a line over which a $(-1,2)$ cycle degenerates where $z_{1}=0=z_{3}, 2 r_{\alpha}+r_{\beta}=t$, and $t \geq r_{\beta} \geq 0$ and finally, iii) There is a line over which $r_{\alpha}=0, t \geq r_{\beta} \geq 0$ where $z_{0}=0=z_{1}$ and $(0,1)$-cycle degenerates. The $U_{1}$ patch is similar, and we end up with the graph for $\mathcal{O}(-3) \rightarrow \mathbf{P}^{2}$ shown in fig. 2 . Since at least two of the $z$ 's have to be zero for the fiber to degenerate, the graph lies in the $r_{\gamma}=0$ plane.

\subsection{Toric algorithm for general geometries}

The above way of constructing $\Gamma$ becomes cumbersome for more complicated geometries. There is an algorithm which does this efficiently. It is a standard construction in toric geometry and we will review it here. This is not meant to be didactical, so for a more thorough exposition see for example [10]. The algorithm is as follows. To each coordinate $z_{i}$ associate a vector $\vec{v}_{i}$ in $\mathbf{Z}^{3}$. The $\vec{v}_{i}$ are chosen to satisfy an equation analogous to (2.4), i.e.

$$
\sum_{i} Q_{i}^{A} \vec{v}_{i}=0
$$

Since the charges $Q^{A}$ are integral, the equations can be solved. The Calabi Yau condition, $\sum_{i} Q_{i}^{A}=0$ implies in fact that we can choose all the vectors to lie on a plane $P$, a unit 
distance from the origin, e.g. we can choose all the $v_{i}$ 's to be of the form $\overrightarrow{v_{i}}=\left(\vec{w}_{i}, 1\right)$, where $\vec{w}_{i}$ is now a two-vector with integer entries. This provides an easy way to partition the $z$ 's into triplets that parameterize $\mathbf{C}^{3}$ patches. Namely, the $z$ 's correspond to a collection of integral points on a plane $P$ whose coordinates are $\vec{w}_{i}$, and this can be triangulated by considering triangles whose vertices are triplets of $\vec{w}$ 's. The triangulation that gives a good covering of $X$ is such that all the triangles in $P$ have unit area. This is in a sense a maximal triangulation. For example, for $\mathbf{C}^{\mathbf{3}}$ we can take: $w_{1}=(0,0), w_{2}=(1,0)$ and $w_{3}=(0,1)$ and the triangulation has a triangle with these vectors as vertices. For $O(-3) \rightarrow \mathbf{P}^{\mathbf{2}}$ we can take $w_{0}=(0,0), w_{1}=(-1,0), w_{2}=(0,-1)$ and $w_{3}=(1,1)$ with 3 triangles corresponding to $\left(w_{0}, w_{1}, w_{2}\right),\left(w_{0}, w_{1}, w_{3}\right)$ and $\left(w_{0}, w_{2}, w_{3}\right)$. In general the choice of the triangulation is not unique. There is an obvious $S L(2, \mathbf{Z})$ action of the plane $P$, which is a symmetry of the closed string theory on $X$. But, in general there are also different possible triangulations of the same set of points. and these correspond to different phases in the Kähler moduli space. For a given choice of the Kähler parameters in (2.4) the allowed triangulation is such that the triplets of coordinates corresponding to every unit-volume triangle can all be simultaneously zero in $X$. We can think about this triangulation as giving rise to a graph $\hat{\Gamma} \in P$. Given $\hat{\Gamma}$ finding the graph $\Gamma$ describing the degeneration of the $T^{2}$ fibers is trivial - it is simply the dual graph in the sense that edges of $\hat{\Gamma}$ are normals to the edges of $\Gamma$ and vice versa!

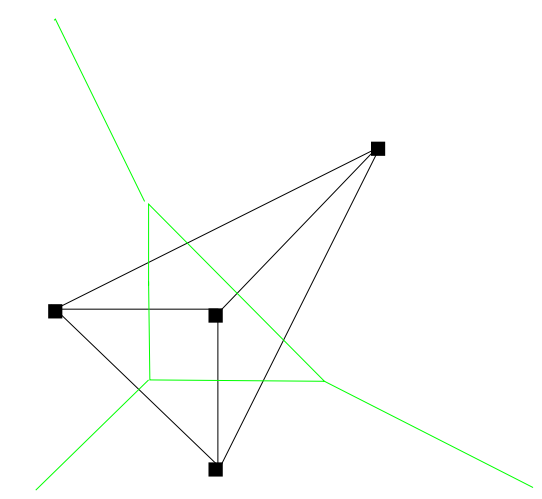

Fig. 3 The graph $\hat{\Gamma}$ of $\mathcal{O}(-3) \rightarrow \mathbf{P}^{\mathbf{2}}$. The black points correspond to vectors $\vec{w}_{i}$. Its dual is the graph of degenerate fibers $\Gamma$.

In fact, running this algorithm backwards, provides a fast way to, associate a CalabiYau manifold to a graph $\Gamma$. We first find a dual graph $\hat{\Gamma}$. The vertices of this graph are vectors $\hat{w}_{i}$, with integer entries. Linear relations between the vectors $\hat{v}_{i}=\left(\hat{w}_{i}, 1\right)$ allow us to read off the charges $Q_{A}^{i}$. 
To completely specify the geometry we also have to specify the Kähler form $\omega \in$ $H^{1,1}(X)$. As discussed above, this is captured by the moduli $t_{A}$ in the D-term equations (2.4) of the linear sigma model. In the formalism we will develop in the following sections we will need to know the areas of holomorphic curves in $X$ which are fixed by the torus actions. It is in fact very easy to determine these directly from the graph $\Gamma$. This however is easiest to physically motivate in terms of the $(p, q)$ five-brane picture which we will discuss below.

\subsection{Semi-compact theories}

In the same spirit, we can also consider certain semi-compact models. Namely, the geometries discussed so far had only a $T^{2}$ subspace of the fiber compact. Here we show that we can also consider some models where four out of six directions in the geometry are compact. These geometries can be obtained by imposing identifications on two of the directions in the base corresponding to the plane of the graph $\Gamma$. Clearly, not all toric Calabi-Yau manifolds will admit the compactifications, but only those with enough symmetry. For those that do, some of the moduli that exist in the non-compact geometry are frozen in the compact one, as they are not consistent with the identifications which we impose.

For example, consider the graph corresponding to $O(-K) \rightarrow \mathbf{P}^{\mathbf{1}} \times \mathbf{P}^{\mathbf{1}}$. When the sizes of the two $\mathbf{P}^{\mathbf{1}}$ 's are equal we can consider a compactification that corresponds to identifying points related by:

$$
\left(r_{\alpha}, r_{\beta}\right) \sim\left(r_{\alpha}+2 \pi R, r_{\beta}+2 \pi R\right) \sim\left(r_{\alpha}-2 \pi R, r_{\beta}+2 \pi R\right)
$$

The resulting geometry has a single Kähler modulus instead of the two that exist in the non-compact case.

As in examples studied in [11] various degenerations of the graph $\Gamma$ and its different phases allowed by the charge conservation (2.1) correspond to geometric transitions of the full Calabi-Yau geometry. It is easy to see that the semi-compact models often have obstructions to existence of transitions that exist in the fully non-compact models. As the simplest example consider the semi-compact version of $T^{*} S^{3}$. This corresponds to having a $(1,0)$ and $(0,1)$ cycle degenerating over the corresponding cycles of the "base" $T^{2}$, at different values of $r_{\gamma}$, i.e. the graph has two components. and corresponding manifold has $b_{3}=1$. This geometry, however, does not have a geometric transition $O(-1) \oplus O(-1) \rightarrow \mathbf{P}^{\mathbf{1}}$ 


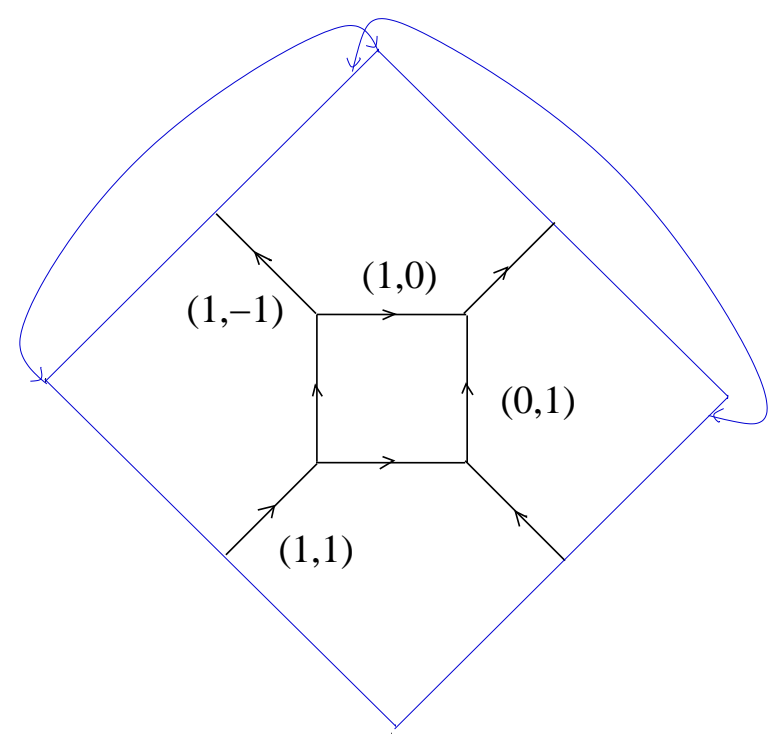

Fig. 4 The graph of the semi-compact $\mathcal{O}(-K) \rightarrow \mathbf{P}^{\mathbf{1}} \times \mathbf{P}^{\mathbf{1}}$ which arises by imposing identifications in the base $\mathbf{R}^{\mathbf{2}}$. The sizes of the two $\mathbf{P}^{\mathbf{1}}$ 's in the base, that are usually independent, must be equal here.

since the blowup-mode that gives the $\mathbf{P}^{\mathbf{1}}$ a finite size is projected out in the semi-compact case. Such an obstruction to a transition from a single $\mathbf{S}^{\mathbf{3}}$ is familiar from the fully compact Calabi-Yau manifolds. Once we discuss the $(p, q)$ five-brane language, it will be manifest that these models have the same obstructions to resolutions of singularities as the compact manifolds do. This is related to the fact that the gauge theories obtained by compactifying string theory on these geometries are honestly 4-dimensional.

\subsection{Relation to $(p, q)$ 5-brane webs}

We can connect the description of Calabi-Yau geometry by a duality to the web of $(p, q)$ fivebranes [12]. This gives an intuitive picture of the geometry. The connection was derived in [13] and we will now review it.

Recall that M-theory on $T^{2}$ is related to type IIB string theory on $\mathbf{S}^{1}$. Since the Calabi-Yau manifolds we have been considering are $T^{2}$ fibered over $B=\mathbf{R}^{4}$, we can relate geometric M theory compactification on Calabi-Yau manifold $X$ to type IIB on flat space $B \times \mathbf{S}^{1}$. However, due to the fact that $T^{2}$ is not fibered trivially, this is not related to the vacuum type IIB compactification. The local type of singularity over a line in the graph is the Taub-Nut space, where the $(p, q)$ label denotes which cycle of the $T^{2}$ corresponds to the $\mathbf{S}^{1}$ of the Taub-Nut geometry. Under the duality, this local degeneration of $X$ is mapped to the $(p, q)$ five-brane that wraps the discriminant locus in the base space $B$, and 
lives on a point on the $\mathbf{S}^{1}$. The fact that the $(p, q)$ type of the five brane is correlated with its orientation in the base is a consequence of the BPS condition. More precisely, a configuration of five branes that preserves supersymmetry and $4+1$ dimensional Lorentz invariance is pointlike in a fixed $\mathbf{R}^{2}$ subspace of the base. In the two remaining directions of the base, which we parameterized by $\left(r_{\alpha}, r_{\beta}\right)$ above, the five branes are lines where the equation of the $(p, q)$ five brane is $p r_{\alpha}+q r_{\beta}=$ const.

The $(p, q)$ five-brane picture provides a simple way to read off the sizes of various holomorphic curves embedded in the Calabi-Yau $X$. For this paper, we will only need to know this for the curves which are invariant under the $T^{2}$ action. It is clear from the discussion in the previous subsections of this section that these are the curves in $X$ which correspond to the edges of the graph $\Gamma$. The duality of M-theory on $X$ to the IIB with 5 -branes relates the membranes wrapping holomorphic curves in $X$ to $(p, q)$ string webs ending on the 5-brane web. The masses of corresponding the BPS states get related by the duality. In the M-theory picture, the masses of BPS states are the Kähler volumes of the holomorphic curves, and in the IIB language they are the tensions $\times$ lengths of the corresponding strings. The curves that project to the edges of the graph correspond to strings that are within the five-branes themselves. These strings are instantons of the five-brane theory. As discussed in [12] the instanton of a $(p, q)$ five brane is a string whose tension is

$$
T_{p, q}=\frac{\operatorname{Im}(\tau)}{|p \tau+q|} T_{s}
$$

where $\tau$ is the type IIB dilaton-axion field $\tau=\frac{\chi}{2 \pi}+\frac{i}{g_{s}}$, and the $T_{s}$ is a tension of a fundamental string which is an instanton in an NS, or $(1,0)$, five-brane. Note that this is not a conventional free $(r, s)$ string tension for any $r, s$ (which would have been $T_{s} \sqrt{r^{2}+q^{2}}$ ), and correspondingly the instanton in general does not correspond to any free $(p, q)$ string. This is because the action of an instanton (i.e. tension in this case, as the instanton is a string) is simply governed by the coefficient of the $F \wedge * F$ term in the five-brane action and this is $T_{s} / g_{s}$ for a D5 brane and $T_{p, q}$ for a $(p, q)$ five-brane. Thus, knowing the length $x$ of a $(p, q)$ edge in $\Gamma$, the area of a holomorphic curve corresponding to this edge is $x / \sqrt{p^{2}+q^{2}}$ (we will take $\tau=i$ which is the square $T^{2}$ ). On the other hand, the slope of the five-brane in the $\mathbf{R}^{\mathbf{2}}$ is correlated with its $(p, q)$ type as we said above, and this allows us to read-off the lengths of all the edges in the graph in terms of the few independent ones which correspond to the Kähler moduli $t^{A}$ in (2.4). For example, suppose that the length of the horizontal edge in the graph of $O(-3) \rightarrow \mathbf{P}^{\mathbf{2}}$ is $t$. Then the length of the 
$(1,-1)$ edge is $t \sqrt{2}$. However the tension of the corresponding instanton in $1 / \sqrt{2}$ so the area of the holomorphic curve corresponding to this leg is $t$. Similarly, we find that the area of the curve corresponding to the $(0,1)$ leg is also $t$.

\subsection{Mirror Symmetry and the dual B-model Geometries}

Mirror manifolds of the local toric Calabi-Yau manifolds were derived in [14], by using T-duality in the linear sigma model in the previous section. The result of [14] is as follows. The mirror theory is a theory of variation of complex structure of a certain hypersurface $Y$ which is given in terms of $n+3$ dual variables $y^{i}$ [14 with the periodicity $y^{i} \sim y^{i}+2 \pi i$. The variables $y^{i}$ are related to variables of the linear sigma model (2.4) as

$$
\operatorname{Re}\left(y^{i}\right)=\left|z^{i}\right|^{2}
$$

so in particular, the D-term equation (2.4) is mirrored by

$$
\sum_{i} Q_{i}^{A} y^{i}=t^{A}
$$

Note that (2.8) has a three-dimensional family of solutions. One parameter is trivial and is given by $y^{i} \rightarrow y^{i}+c$. Let us parameterize the two non-trivial family of solutions by $u, v$, and pick an inhomogenous solution. The the hypersurface $Y$ is given by [15]

$$
x \tilde{x}=e^{y_{1}(u, v)}+e^{y_{2}(u, v)} \ldots+e^{y_{N+3}(u, v)} \equiv P(u, v),
$$

where $y_{i}(u, v)$ solve (2.8). The solutions to (2.8) are of the form

$$
y_{i}=w_{i}^{u} u+w_{i}^{v} v+t_{i}(t)
$$

for some vector $\vec{w}_{i}=\left(w_{i}^{u}, w_{i}^{v}\right)$ with integer entries. In fact this is the same vector that we associated to the coordinate $z_{i}$ in the previous section, when we discussed toric geometry. The monomials $e^{w_{1} u+w_{2} v}$ are in one to one correspondence with points of the graph $\hat{\Gamma}$.

In the sections to follow a prominent role will be played by the Riemann surface $\Sigma_{X}$, obtained by setting $x, \tilde{x}$ to zero in (2.9).

$$
\Sigma_{X}: 0=P(u, v)
$$

Note that this Riemann surface is closely related to the graph $\Gamma$ and it is in fact obtained by the fattening of its edges. For example, for the mirror of $\mathbf{C}^{\mathbf{3}}$ we get

$$
e^{u}+e^{v}+1=0
$$


and this has three asymptotic regimes corresponding to $u \rightarrow \infty$ where the equation of the Riemann surface is $v=i \pi$. This is a long cylinder parameterized by $u$. Similarly, there is a long cylinder parameterized by $v \rightarrow \infty$ where $u=i \pi$ and there is a third cylinder where $u=v+i \pi$, and $u, v \rightarrow \infty$, so that this Riemann surface corresponds to a sphere with three punctures. From "far away" the Riemann surface will look like the graph $\Gamma$ of $\mathbf{C}^{\mathbf{3}}$. Similarly, the Riemann surface $\Sigma_{X}$ of any $X$ has a degenerate limit where it looks like the graph $\Gamma$. It is clear that by gluing various patches given by (2.11) dictated by the graph $\Gamma$ we can obtain the full Riemann surface $\Sigma_{X}$.

\section{Topological A-model and the vertex}

The amplitudes of topological A-model localize on holomorphic maps from the worldsheet to the target space [1]. In particular the path integral defining the free energy of the theory reduces to a sum over the topological type of holomorphic maps from the worldsheet to the Calabi-Yau space $X$. Each term in the sum involves an integral over the moduli space $\mathcal{M}$ of that type of maps, which leads to the so called Gromov-Witten invariant of that map, weighted by the $e^{- \text {Area }}$, where the area is the one of the target space curve.

In this paper we will find a very efficient way to calculate the A-model amplitudes on

local toric Calabi-Yau manifolds described in the previous section, to all genera, exactly. The rough idea is to place Lagrangian D-brane/anti-D-brane pairs in appropriate places (one on each edge of the toric diagram) to cut the Calabi-Yau manifold $X$ into patches which are $\mathbf{C}^{3}$. They do not quite cut the Calabi-Yau in pieces as their dimension is too low, but all closed string worldsheet configurations will nevertheless cross them. More precisely using toric actions the configurations can be made to pass through the lines of the toric graph [16]. Thus if we are interested in the closed string amplitudes we could use the D-branes as "tags" for when the closed string goes from one patch to another. Thus the open string amplitudes on each patch, glued together in an appropriate way, should have the full information about the closed string amplitudes. 


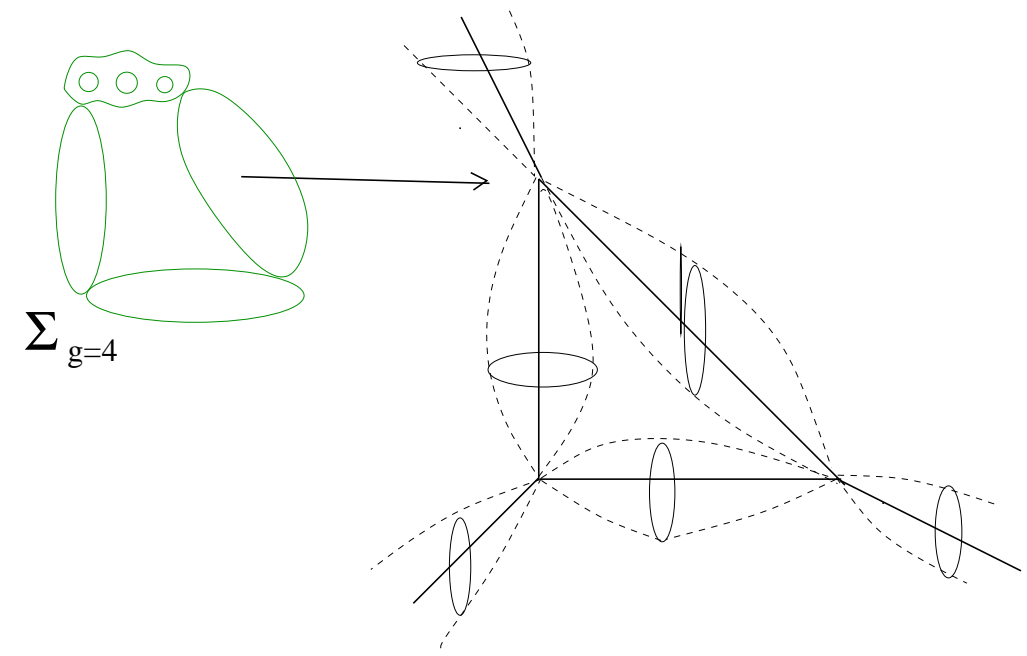

Fig. 5 The curves that are invariant under $T^{2}$ action in the $\mathcal{O}(-3) \rightarrow \mathbf{P}^{\mathbf{2}}$ geometry. All the invariant curves are $\mathbf{P}^{\mathbf{1}}$ 's. The maps from $\Sigma_{g>0}$ that give a non-zero contribution to the A-model amplitudes are degenerate maps where the genus $g>0$ parts of the curve are mapped to vertices.

The idea is then as follows. Consider chopping the graph $\Gamma$ into tri-valent vertices by cutting each of the legs into two. Physically we can view this as placing a D-brane/anti-Dbrane pair. Each vertex corresponds to a $\mathbf{C}^{3}$ patch, as in figs. 2,5. This cuts the $\mathbf{P}^{\mathbf{1}}$ 's which correspond to compact legs of $\Gamma$ into disks. Consider the maps $\Sigma_{g} \rightarrow X$. The maps which contribute to the A-model amplitudes themselves project to (subgraph of) $\Gamma$, and cutting the graphs cuts the maps as well, so we get Riemann surfaces with boundaries. We are led to consider open topological string on $\mathbf{C}^{3}$ with three (stacks of) Lagrangian D-branes of the appropriate kind, one on each leg. From these data we should be able to obtain, by suitable gluing, closed string amplitudes on arbitrary toric Calabi-Yau threefolds.

\subsection{The vertex as an open string amplitude}

Consider again the description of the $\mathbf{C}^{3}$ in section 2. The Lagrangian D-branes we need are in fact among the original examples of special Lagrangians of Harvey and Lawson [17]. The topology of all of the Lagrangians is $\mathbf{C} \times \mathbf{S}^{1}$. In particular, they project to lines in the base $\mathbf{R}^{3}$, and wrap the $T^{2}$ fiber. In the base, the three Lagrangians $L_{1,2,3}$ are given 
by 3

$$
\begin{aligned}
& L_{1}: \quad r_{\alpha}=0, \quad r_{\beta}=r_{1}^{*}, \quad r_{\gamma} \geq 0 \\
& L_{2}: \quad r_{\beta}=0, \quad r_{\alpha}=r_{2}^{*}, \quad r_{\gamma} \geq 0 \\
& L_{3}: \quad r_{\alpha}-r_{\beta}=0, \quad r_{\alpha}=r_{3}^{*}, \quad r_{\gamma} \geq 0 .
\end{aligned}
$$

In order not to have the boundary at $r_{\gamma}=0, L_{i}$ 's are constrained to end on the graph $\Gamma$, where one of the 1-cycles of the $T^{2}$ degenerates to $\mathbf{S}^{1}$. The parameters $r_{i}^{*}$ correspond to the moduli of $L_{i}$ 's, and the "no boundary" constraint that we just mentioned is what constrains the number of the moduli to one. The Lagrangians are easily seen to intersect the fixed $\mathbf{P}^{1}$ 's along $\mathbf{S}^{1}$ 's so the boundaries of the maps can end on them. For example, a holomorphic disc ending on $L_{1}$ is given by $z_{1}=0=z_{3},\left|z_{2}\right|^{2} \leq r_{1}^{*}$.

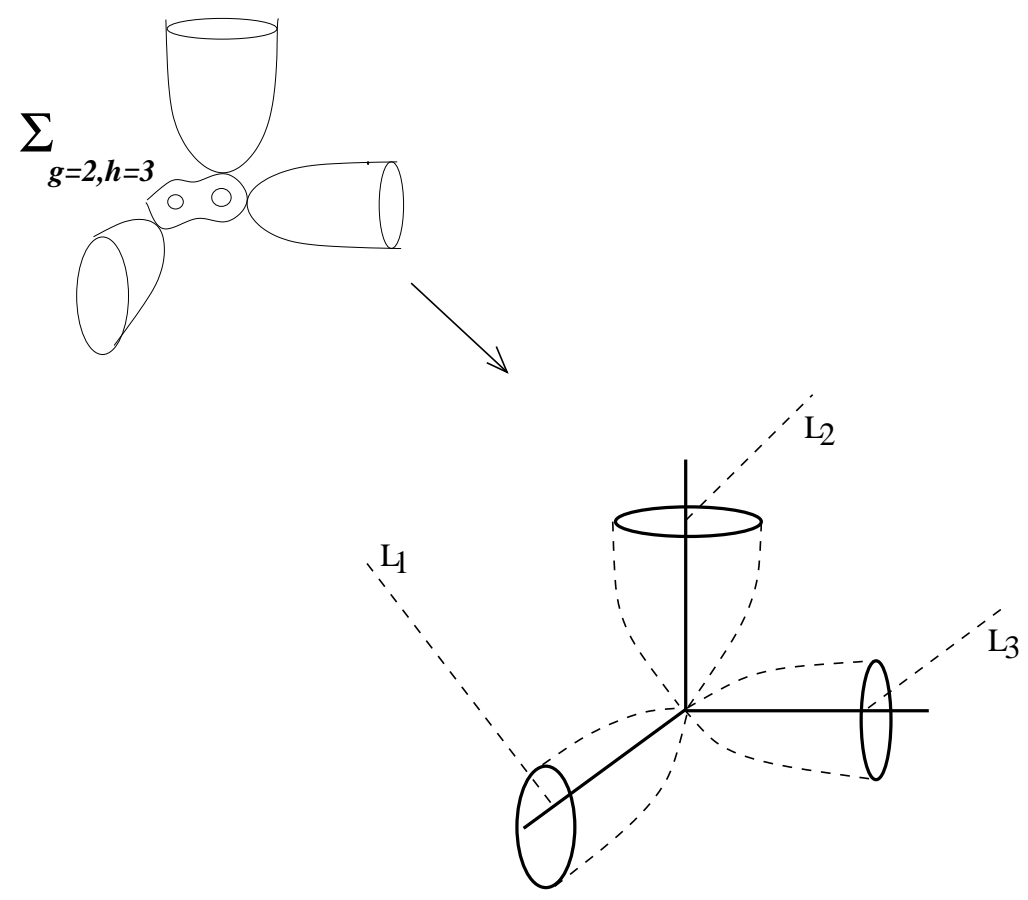

Fig. 6 A $\mathbf{C}^{3}$ with three-stacks of Lagrangian D-branes of the type discussed in the text. The A-model amplitudes localize on holomorphic maps with boundaries where all the higher genus information is mapped to the vertex.

Now, consider the topological A-model string amplitude corresponding to some number of D-branes $N_{i}$ on the $i$-th Lagrangian $L_{i}$ on $\mathbf{C}^{3}$. The partition function takes the

3 The Lagrangians are pointlike in the fiber generated by $r_{\gamma}$. The fiber is parameterized by $\operatorname{Re}\left(z_{1} z_{2} z_{3}\right)$ and the Lagrangians are where this vanishes. 
form

$$
Z=\sum_{\vec{k}^{(1)}, \vec{k}^{(2)}, \vec{k}^{(3)}} C_{\vec{k}^{(1)}, \vec{k}^{(2)}, \vec{k}^{(3)}} \prod_{i=1}^{3} \frac{1}{z_{\vec{k}^{(i)}}} \operatorname{Tr}_{\vec{k}^{(i)}} V_{i}
$$

where $V_{i}$ is the path ordered exponential of the Wilson-line on the $i$-th D-brane, $V_{i}=$ $\mathrm{P} \exp \left[\oint A_{1}\right]$ around the $\mathbf{S}^{1}$,

$$
\operatorname{Tr}_{\vec{k}} V=\prod_{j=1}^{\infty}\left(\operatorname{tr} V^{j}\right)^{k_{j}}
$$

and

$$
z_{\vec{k}}=\prod_{j} k_{j} ! j^{k_{j}} .
$$

Note that there are $k_{j}$ holes of winding number $j$ so the sum $h=|\vec{k}|=\sum_{j} k_{j}$ is the total number of holes on a fixed D-brane, and $\ell=\sum_{j} j k_{j}$ is the total winding number. We have absorbed the modulus of the Lagrangian into the corresponding $V$ which is complexified in string theory. The vertex amplitude, $C_{\vec{k}^{(1)}, \vec{k}^{(2)}, \vec{k}^{(3)}}$ is naturally a function of the string coupling constant $g_{s}$ and, in the genus expansion, it contains information about maps from Riemann surfaces of arbitrary genera into $\mathbf{C}^{3}$ with boundaries on the D-branes, see fig. 6 .

The vertex $C$ is the basic object from which, by gluing, we should be able to obtain closed string amplitudes on arbitrary toric geometries. As we will see later, the vertex is naturally used to calculate general A-model amplitudes with boundaries as well.

\subsection{Framing of the vertices}

Because of the above considerations we are led to consider non-compact D-branes in $\mathbf{C}^{3}$. Due to the non-compactness of the world-volume of D-branes, to fully specify the quantum theory we must specify the boundary conditions on the fields on the D-branes at infinity. This was discovered in [11] and is the closed string dual to the framing ambiguity of the Chern-Simons amplitudes [18].

To keep track of the boundary condition at infinity, we can use the following trick [19]. We modify the geometry in a way that makes the Lagrangian cycles wrapped by the D-branes compact, while not affecting the topological A-model amplitudes. We do so by introducing compact $\mathbf{S}^{3}$ cycles in the geometry by allowing the $T^{2}$ fiber to degenerate at additional locations in the base $\mathbf{R}^{\mathbf{3}}$, as in the figure 7 . The additional three lines $F_{i}$ in the base correspond to degeneration of a fixed $f_{i}=\left(p_{i}, q_{i}\right)$ cycle there. There are now

compact special Lagrangian $\mathbf{S}^{3}$ cycles $\tilde{L}_{1,2,3}$ which correspond to paths of the shortest 
distance between the graphs $\Gamma$ and $F_{i}$. For this cycle to be a non-degenerate $\mathbf{S}^{3}$ we need the following condition on the holonomy

$$
f_{i} \wedge v_{i}=1
$$

where $v_{i}$ corresponds to the $H_{1}\left(T^{2}\right)$ class of the edges of the graph. Note that we have chosen a particular orientation for the framing so that the above product is always +1 . Clearly, if $f_{i}$ is a solution to (3.3), so is $f_{i}-n v_{i}$ for any integer $n$. This $\mathbf{Z}$ valued choice does affect the physics of the D-brane. To specify the theory on the D-brane fully, we must specify a choice of framing [11], i.e. a choice of the integer $n$. This is a quantum ambiguity and only the relative values of $n$ are meaningful. Given an (arbitrary) choice of framing for the i-th leg, i.e. a vector $f_{i}^{(0)}$, the vector $f^{(n)}$ corresponds to a relative framing associated to an integer $n$ if

$$
f^{(n)} \wedge f^{(0)}=n
$$

It is crucial for us to keep track of framing. The relevant object is a framed vertex,

$$
C_{\vec{k}^{(1)}, \vec{k}^{(2)}, \vec{k}^{(3)}}^{\left(f_{1}, f_{2}, f_{3}\right)}
$$

where we specify the framing of the D-branes on the three legs.

Without loss of generality we can take the $v_{i}$ to be $v_{1}=(-1,-1), v_{2}=(0,1), v_{3}=$ $(1,0)$, since any other choice is related to this one by an $S L(2, \mathbf{Z})$ transformation. More generally we can introduce a vertex which depends on both $v_{i}$ and $f_{i}$, but knowing the vertex for the canonical choice of $v_{i}$ with arbitrary framing $f_{i}$ is enough. Moreover, if we know the vertex in any one framing, the vertex in any other framing is related to it in a simple way [11,20]. In order to describe this it is most convenient to go to the "representation basis" for the vertex which we will now turn to.

\subsection{The vertex in the representation basis}

Topological open string amplitudes can be written in terms of products of traces to various powers, as in (3.2). They can also be rewritten in the representation basis, and this can be done unambiguously in the limit where we take $N_{i} \rightarrow \infty$ branes. We define the representation basis for the vertex by

$$
\sum_{R_{1}, R_{2}, R_{3}} C_{R_{1}, R_{2}, R_{3}}^{f_{1}, f_{2}, f_{3}} \prod_{i=1}^{3} \operatorname{Tr}_{R_{i}} V_{i}=\sum_{\vec{k}^{(1)}, \vec{k}^{(2)}, \vec{k}^{(3)}} C_{\vec{k}^{(1)}, \vec{k}^{(2)}, \vec{k}^{(3)}}^{f_{1}, f_{2}, f_{3}} \prod_{i=1}^{3} \frac{1}{z_{\vec{k}^{(i)}}} \operatorname{Tr}_{\vec{k}^{(i)}} V_{i}
$$


To obtain $C$ in the representation basis defined above, we make use of Frobenius formula

$$
\operatorname{Tr}_{\vec{k}} V=\sum_{R} \chi_{R}(C(\vec{k})) \operatorname{Tr}_{R} V
$$

where $\chi_{R}(C(\vec{k}))$ is the character of the symmetric group $S_{\ell}$ of $\ell$ letters for the conjugacy class $C(\vec{k})$, in representation corresponding to the Young tableau of $R$. Using this we obtain

$$
C_{R_{1}, R_{2}, R_{3}}^{f_{1}, f_{2}, f_{3}}=\sum_{\vec{k}^{(1)}, \vec{k}^{(2)}, \vec{k}^{(3)}} C_{\vec{k}^{(1)}, \vec{k}^{(2)}, \vec{k}^{(3)}}^{f_{1}, f_{2}, f_{3}} \prod_{i} \frac{\chi_{R_{i}}\left(C\left(\vec{k}^{(i)}\right)\right)}{z_{\vec{k}^{(i)}}}
$$

Now we are ready to describe the framing dependence of the vertex. We have [20]

$$
C_{R_{1}, R_{2}, R_{3}}^{f_{1}-n_{1} v_{1}, f_{2}-n_{2} v_{2}, f_{3}-n_{3} v_{3}}=(-1)^{\sum_{i} n_{i} \ell\left(R_{i}\right)} q^{\sum_{i} n_{i} \kappa_{R_{i}} / 2} C_{R_{1}, R_{2}, R_{3}}^{f_{1}, f_{2}, f_{3}},
$$

where $\kappa_{R}$ is related to the quadratic Casimir $C_{R}$ of the representation $R$ of $U(N)$ as $\kappa_{R}=C_{R}-N \ell(R)$, and $\ell(R)$ is the number of boxes of the representation (which is the same as the total winding number in the $\vec{k}$-basis). If the representation $R$ is associated to a Young tableaux whose $i$-th row has $\ell_{i}$ boxes, $\ell(R)=\sum_{i} \ell_{i}$ one has

$$
\kappa_{R}=\sum_{i} \ell_{i}\left(\ell_{i}-2 i+1\right)
$$

\subsection{Symmetries of the vertex}

Consider an $S L(2, \mathbf{Z})$ transformation that acts on the $T^{2}$ fiber of $\mathbf{C}^{3}$, in the presence of D-branes. As already noted the vertex depends on three pairs $\left(f_{i}, v_{i}\right)$ where $v_{i}$ denotes the $(p, q)$ structure of the edge and $f_{i}$ denotes the framing associated to that edge, and one has

$$
f_{i} \wedge v_{i}=1
$$

which means that $\left(f_{i}, v_{i}\right)$ forms an oriented basis for $H_{1}\left(T^{2}\right)$. Moreover, if we orient the edges inward towards the vertex, then $\sum_{i} v_{i}=0$. One also has that $v_{i} \wedge v_{j}= \pm 1$ for $i \neq j$. We can choose a cyclic ordering of $v_{i}$ according to the embedding of the corresponding vectors in $\mathbf{R}^{2}$. In terms of this cyclic ordering we have

$$
v_{2} \wedge v_{1}=v_{1} \wedge v_{3}=v_{3} \wedge v_{2}=1
$$

It is clear that an element $g \in S L(2, \mathbf{Z})$ generates a symmetry of the vertex while replacing

$$
\left(f_{i}, v_{i}\right) \rightarrow\left(g \cdot f_{i}, g \cdot v_{i}\right)
$$




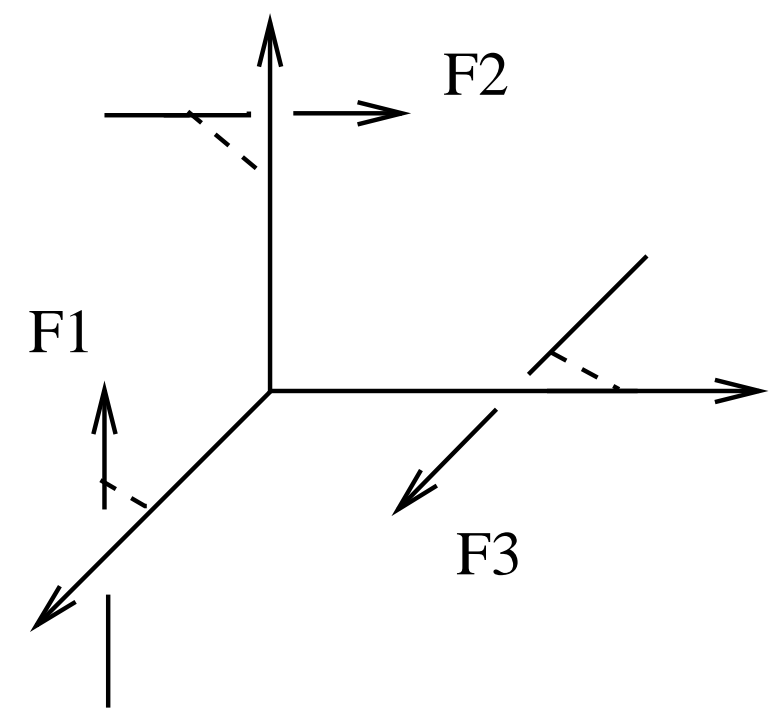

Fig. 7 Three stacks of D-branes on $\mathbf{C}^{3}$. We have introduced graphs $F_{1,2,3}$ to help us keep track of framing. $F_{i}$ are straight lines in the base, corresponding to vectors $f_{i}$ in the text. Different choices of $f_{i}$ give different amplitudes. The choice in this figure is defined to be canonical framing.

There is one particularly natural choice of framing $f_{i}$ based on symmetry considerations, namely (see fig. 7)

$$
\left(f_{1}, f_{2}, f_{3}\right)=\left(v_{2}, v_{3}, v_{1}\right)
$$

Note that this has the required property that $f_{i} \wedge v_{i}=1$. For any given choice of $v_{i}$ cyclically ordered in this way, we shall call this the canonical framing and denote the corresponding vertex by $C$. Any other choice of framing, relative to this canonical choice, will be denoted by $C^{n_{1}, n_{2}, n_{3}}$ where $n_{i}$ denote the amount of change in framing relative to the canonical choice. Let $C_{R_{1}, R_{2}, R_{3}}$ denote the vertex for the canonical framing for $v_{i}:(-1,-1),(0,1),(1,0)$. Then it follows that

$$
C_{R_{1}, R_{2}, R_{3}}^{\left(f_{i}, v_{i}\right)}=(-1)^{\sum_{i} n_{i} \ell\left(R_{i}\right)} q^{\frac{1}{2} \sum_{i} n_{i} \kappa_{R_{i}}} C_{R_{1}, R_{2}, R_{3}}
$$

where

$$
n_{i}=f_{i} \wedge v_{i+1}
$$

and $i$ runs mod 3. With three D-branes on the legs of the vertex, the vertex amplitude $C_{R_{1}, R_{2}, R_{3}}$ is invariant under the $\mathbf{Z}_{3}$ subgroup of $S L(2, \mathbf{Z})$ taking

$$
v_{1} \rightarrow v_{2}, \quad v_{2} \rightarrow v_{3}, \quad v_{3} \rightarrow v_{1}
$$


Note that the condition that $v_{3} \rightarrow v_{1}$ follows from the first two from $\sum_{i} v_{i}=0$. Clearly there is such an $S L(2, \mathbf{Z})$ transformation, because $\left(v_{1}, v_{2}\right)$ and $\left(v_{2}, v_{3}\right)$ form an oriented basis for $H_{1}\left(T^{2}\right)$. For example for the simple choice of $v_{i}:(-1,-1),(0,1),(1,0)$ it is generated by $T S^{-1}$ in the standard basis for generators of $S L(2, \mathbf{Z})$, so we see that the vertex amplitude with canonical choice of framing, which is compatible with this cyclicity, has a cyclic symmetry,

$$
C_{R_{1}, R_{2}, R_{3}}=C_{R_{3}, R_{1}, R_{2}}=C_{R_{2}, R_{3}, R_{1}} .
$$

So far we have oriented edges of the vertex away from the vertex. In gluing vertices together we would need also to deal with arbitrary orientation of the edges. Suppose for example we take $v_{1} \rightarrow-v_{1}$. What this does is to change the orientation of the circle on the corresponding D-brane. This is a parity operation on the D-brane, which changes the action to minus itself. Thus a genus $g$ topological string amplitude with $h$ boundaries on the corresponding D-brane (in the 't Hooft notation) gets modified by

$$
(-1)^{\text {loops }}=(-1)^{2 g-2+h}=(-1)^{h}
$$

This can also be obtained by viewing the change of the sign of the action as replacing a topological brane by a topological anti-brane which replaces $N \rightarrow-N$ [21]. It is convenient to write how this modifies the vertex in the representation basis. This can be done using

$$
\chi_{Q^{t}}(C(\vec{k}))=(-1)^{|\vec{k}|+\ell(Q)} \chi_{Q}(C(\vec{k})),
$$

where $\ell(Q)$ denotes the number of boxes of representation $Q$. It follows that

$$
C_{R_{1}, R_{2}, R_{3}} \rightarrow v_{1} \rightarrow-v_{1}(-1)^{\ell\left(R_{1}\right)} C_{R_{1}^{t}, R_{2}, R_{3}} .
$$

Similarly we can change any of the other $v_{i} \rightarrow-v_{i}$. 


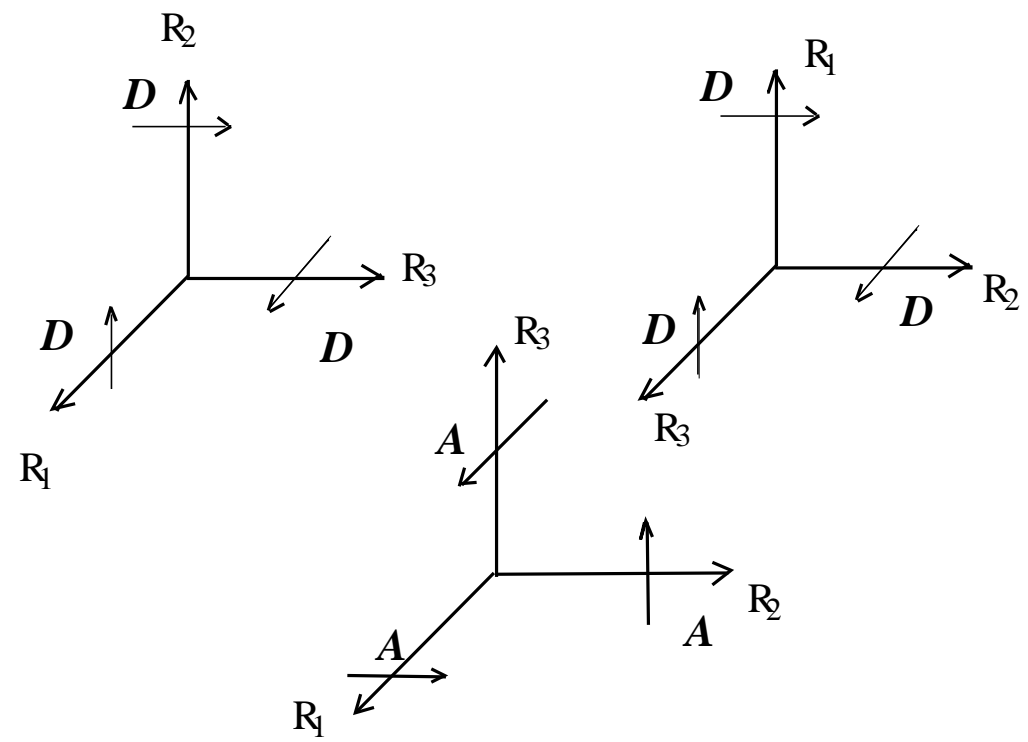

Fig. 8 Various symmetries of the three-point vertex. The figures in the top row are related by a $\mathbf{Z}_{3}$ subgroup of $S L(2, \mathbf{Z})$. The figure in the bottom row is generated from the top left one by a symmetry of $\mathbf{C}^{3}$ that exchanges $z_{1}$ and $z_{2}$. This also maps a D-brane $(D)$ to an anti D-brane $(A)$.

We have seen that the vertex has cyclic symmetry in the canonical framing. It is natural to ask what symmetry it has under permutation of any of the two representations. There is a symmetry of $\mathbf{C}^{3}$ that exchanges any pair of its coordinates, say $z_{1}, z_{2}$. This acts as orientation reversal on the world-volume of all three D-branes, as it acts on the $T^{2}$ fiber by exchanging $(1,0)$ and $(0,1)$-cycles, and the $T^{2}$ is wrapped by all the D-branes. In addition the framings are shifted by one unit: We have

$$
\left(\left(f_{1}, v_{1}\right),\left(f_{2}, v_{2}\right),\left(f_{3}, v_{3}\right)\right) \rightarrow\left(\left(f_{2}, v_{1}\right),\left(f_{3}, v_{2}\right),\left(f_{1}, v_{3}\right)\right)
$$

and from (3.8) it follows that the new framing is shifted by

$$
\left(f_{2} \wedge v_{2}, f_{3} \wedge v_{3}, f_{1} \wedge v_{1}\right)=\left(v_{3} \wedge v_{2}, v_{1} \wedge v_{3}, v_{2} \wedge v_{1}\right)=(1,1,1)
$$

(see also fig. 8). From this it follows that

$$
C_{R_{1}, R_{2}, R_{3}}=(-1)^{\sum_{i} \ell_{i}} C_{R_{1}^{t}, R_{3}^{t}, R_{2}^{t}}^{-1,}
$$

Since $\kappa_{R^{t}}=-\kappa_{R}$, we can write this as

$$
C_{R_{1}, R_{2}, R_{3}}=q^{\sum_{i} \kappa_{R_{i}} / 2} C_{R_{1}^{t}, R_{3}^{t}, R_{2}^{t}}
$$




\subsection{Gluing the vertices}

In this section, we discuss how to glue open string amplitudes to obtain closed string amplitudes. Consider a leg of some graph $\Gamma$, as in the figure 3 . The leg will contribute to closed string amplitudes via holomorphic curves that map to the corresponding $\mathbf{P}^{\mathbf{1}}$. By cutting the curve in the middle of the leg, we obtain a product of open string amplitudes. Clearly, connected closed string graphs can give open string graphs that are disconnected, so the gluing must be done at the level of the partition function, schematically,

$$
Z(\Gamma) \sim Z\left(\Gamma_{L}\right) \times Z\left(\Gamma_{R}\right)
$$

where $Z(\Gamma)$ is the amplitude corresponding to the graph $\Gamma$ and, by cutting one of its legs, the graph can be decomposed into $\Gamma_{L}$ and $\Gamma_{R}$.

Moreover, clearly the open string Riemann surfaces one gets in this way have matching number of holes on the leg over which we glue, and also the winding numbers. Thus, the right hand side of the above equation is in fact

$$
\sum_{\vec{k}} Z\left(\Gamma_{L}\right)_{\vec{k}} \frac{\exp (-\ell(\vec{k}) t)}{\prod_{j} k_{j} ! j^{k_{j}}} Z\left(\Gamma_{R}\right)_{\vec{k}}
$$

Above, $t$ is the size of the relevant $\mathbf{P}^{\mathbf{1}}$. In gluing these we have to be careful that both gluing branes are defined with respect to the same framing. The combinatorial factor comes about because all holes with the same winding number are indistinguishable, and the factor of $j$ for each hole of winding number $j$ comes as the gluing respects the cyclic ordering of the $j$ windings. In addition, we must remember that $X_{L}$ and $X_{R}$ (the manifolds corresponding to the graphs $\left.\Gamma_{L, R}\right)$ come equipped with a choice of complex structures, and this induces natural orientation of boundaries of the two disks in $X_{L, R}$. In order to glue the two disks into a $\mathbf{P}^{\mathbf{1}}$ their boundaries must be oriented oppositely, which can be interpreted as putting branes versus anti-branes. As was already discussed this is equivalent to multiplying the amplitude by $(-1)^{h}$ where $h$ is the number of boundaries of the Riemann surface. This gives the gluing a nice physical interpretation: we put $\mathrm{N}$ D-branes on the relevant leg in $X_{L}$ and $N$ anti D-branes in $X_{R}$. The D-branes annihilate, so from the corresponding open string amplitudes we obtain the amplitude for closed strings on $X$. To summarize,

$$
Z(\Gamma)=\sum_{\vec{k}} Z\left(\Gamma_{L}\right)_{\vec{k}} \frac{(-1)^{|\vec{k}|} e^{-\ell(\vec{k}) t}}{z_{\vec{k}}} Z\left(\Gamma_{R}\right)_{\vec{k}}
$$


Obviously, (3.13) holds even in the presence of D-branes in $X$, where $Z(X)$, etc. refer to amplitudes with D-branes. At the very least, this is true, as long as the D-branes are at locations away from the relevant leg, as all the considerations that led to (3.13) are purely local. We will return to this in the sections below.

Note that in the representation basis the gluing operation is simply:

$$
Z(X)=\sum_{Q} Z\left(X_{L}\right)_{Q}(-1)^{\ell_{Q}} e^{-\ell(Q) t} Z\left(X_{R}\right)_{Q^{t}}
$$

which follows from (3.10) and orthonormality of the characters

$$
\sum_{\vec{k}} \frac{1}{z_{\vec{k}}} \chi_{R}(C(\vec{k})) \chi_{R^{\prime}}(C(\vec{k}))=\delta_{R R^{\prime}}
$$

\subsection{The gluing algorithm for closed and open strings}

Putting together all we have said so far, we can summarize the rules for computing closed string amplitudes from the cubic vertex and the gluing rules as follows:

i) From the toric data described in the section 2, we can find the graph $\Gamma$ corresponding to the loci where $T^{2}$ fibration degenerates. The edges of the graph are labeled by integral vectors $v_{i}$ that encode which cycle of the $T^{2}$ fiber degenerates over the $i$-th edge. To each edge associate a representation $R_{i}$.

ii) For smooth Calabi-Yau, the graph can be partitioned to trivalent vertices and corresponding $\mathbf{C}^{3}$ patches $U_{a}$, where $a$ labels the vertices $a=1,2, \ldots$

iii) This associates to every vertex an ordered triplet of vectors $\left(v_{i}, v_{j}, v_{k}\right)$ by reading off the three edges that meet at the vertex in a counter-clockwise cyclic order $-\left(v_{i}, v_{j}, v_{k}\right)$ is equivalent to $\left(v_{j}, v_{k}, v_{i}\right)$.

iv) If all the edges are incoming, we associate a factor $C_{R_{i}, R_{j}, R_{k}}$ to the vertex $U_{a}$, otherwise we replace the corresponding representation by its transpose times $(-1)^{\ell(R)}$. 
v) Let the vertex $U_{a}$ share the $i$-th edge with the vertex $U_{b}$ whose corresponding triple is $\left(v_{i}, v_{j}^{\prime}, v_{k}^{\prime}\right)$. We can assume $v_{i}$ is outgoing at $U_{a}$ and ingoing at $U_{b}$. We glue the amplitudes by summing over the representations on the $i$-th edge as:

$$
\sum_{R_{i}} C_{R_{j} R_{k} R_{i}} e^{-\ell\left(R_{i}\right) t_{i}}(-1)^{\left(n_{i}+1\right) \ell\left(R_{i}\right)} q^{-n_{i} \kappa_{R_{i}} / 2} C_{R_{i}^{t} R_{j}^{\prime} R_{k}^{\prime}}
$$

where the integer $n_{i}$ is defined as

$$
n_{i}=\left|v_{k}^{\prime} \wedge v_{k}\right|
$$

and $\left|v_{k}{ }^{\prime} \wedge v_{k}\right|$ equals $v_{k}{ }^{\prime} \wedge v_{k}$ if both $v_{k}$ and $v_{k}^{\prime}$ are in(out)going, and $-v_{k}{ }^{\prime} \wedge v_{k}$, otherwise. This factor reflects the fact that the framing over the $i$-th edge should be the same on the two side of gluings. The sign $(-1)^{\left(n_{i}+1\right) \ell\left(R_{i}\right)}$ in $(3.15)$ comes from the sign associated to the framing, and the one associated to the gluing in (3.14).

vi) From the D-term equations (2.4), or the $(p, q)$ 5-brane diagrams read off the lengths $t_{i}$ of the edges in terms of the Kähler moduli $t^{A}$ of $X, t_{i}=t_{i}\left(t^{A}\right)$. Note that the edges of the graph $\Gamma$ are straight lines on the plane, with rational slope. To the $i$-th edge in the $\left(p_{i}, q_{i}\right)$ direction of length $x_{i}$ in the plane, we associate a Kähler parameter $t_{i}=x_{i} / \sqrt{p^{2}+q^{2}}$.

vii) For a non-compact edge of the graph $\Gamma$ the corresponding representation $R$ is necessarily trivial, $R=0$ (we will sometimes denote this also by $R=\cdot$ ).

Naturally, the vertex can be used for calculating open string amplitudes on toric Calabi-Yau manifolds as well as the closed string ones. When we place the D-branes on the non-compact, outer edges of the graph $\Gamma$, we simply modify the (vii) above to a sum over arbitrary representations $R$ on the edge, where we weight the representations by $\operatorname{Tr}_{R} V$ and $V$ is the holonomy on the corresponding D-brane. For D-branes on the inner edges, when we glue the maps on $X$ from the maps on $X_{L}$ and $X_{R}$, we must allow for maps with the boundaries on the D-brane. Suppose that the we wish to calculate an amplitude corresponding to a D-brane on the $i$-th edge of the toric graph. This modifies the gluing rule in (v) above as follows. 
v') For a single D-brane on the i-th edge, which is shared by vertices $U_{a}$ and $U_{b}$ in the setup of (v), we glue the amplitudes by summing over the representations on the $i$-th edge and representations $Q_{i}^{L}, Q_{i}^{R}$ which "stop" on the D-brane from left and right as:

$$
\sum_{R_{i}, Q_{i}^{L}, Q_{i}^{R}} C_{R_{j}, R_{k}, R_{i} \otimes Q_{i}^{L}}(-1)^{s(i)} q^{f(i)} e^{-L(i)} C_{R_{i}^{t} \otimes Q_{i}^{R}, R_{j}^{\prime}, R_{k}^{\prime}} \operatorname{Tr}_{Q_{i}^{L}} V_{i} \operatorname{Tr}_{Q_{i}^{R}} V_{i}^{-1}
$$

where we have collected the length, framing and sign factors in functionals $L(i), f(i)$ and $s(i)$ on this leg:

$$
\begin{gathered}
L(i)=\ell\left(R_{i}\right) t_{i}+\ell\left(Q_{i}^{L}\right) r_{i}+\ell\left(Q_{i}^{R}\right)\left(t_{i}-r_{i}\right) \\
f(i)=p_{i} \kappa_{R_{i} \otimes Q_{i}^{L}} / 2+\left(n+p_{i}\right) \kappa_{R_{i}^{t} \otimes Q_{i}^{R}} / 2 . \\
s(i)=\ell\left(R_{i}\right)+p_{i} \ell\left(R_{i} \otimes Q_{i}^{L}\right)+\left(n+p_{i}\right) \ell\left(R_{i}^{t} \otimes Q_{i}^{R}\right) .
\end{gathered}
$$

The piece of the edge to the left of the brane has length $r_{i}$, while the right-hand side of the edge has length $t_{i}-r_{i} . V_{i}$ is the holonomy on the D-brane. Note that $e^{-r}$ naturally complexifies $V_{i}$ : changing the holonomy by $V_{i} \rightarrow V_{i} e^{i \theta}$ changes $\operatorname{Tr}_{R} V_{i}$ to $e^{i \ell(r) \theta} \operatorname{Tr}_{R} V$. The appearance of both $\operatorname{Tr}_{R} V_{i}$ and $\operatorname{Tr}_{R} V_{i}^{-1}$ reflects the fact that, along with open string instantons of area $r$ and charge +1 ending on the D-brane, there are those of area $t-r$ and charge -1 , where the "charge" refers to how their boundaries couple to the holonomy on the D-brane world volume. The integer $n_{i}$ is defined as in (v) and the choice of an integer $p$ corresponds to a choice of framing. This way of incorporating framing is natural. Namely, while for the closed string amplitudes only the relative framing in the left vs. right patch matters, corresponding to $n_{i}=\left|v_{k}^{\prime} \wedge v_{k}\right|$, for the open string the absolute choice of framing matters: we pick a vector $f_{i}$, which frames the $i$ 't leg both for the left and the right patch so that $f_{i} \wedge v_{i}=1$. This corresponds to the choice of coordinate on the D-brane which does affect the open string amplitude. Then the framing of the left and the right vertex are

$$
p_{i}=\left|f_{i} \wedge v_{k}\right|, \quad n_{i}+p_{i}=\left|f_{i} \wedge v_{k}^{\prime}\right|,
$$

where $\left|f_{i} \wedge v_{k}\right|$ is $f_{i} \wedge v_{k}$ if $v_{k}$ and $v_{i}$ are both in(out) going in the vertex $U_{a}$, and equals $-f_{i} \wedge v_{k}$ otherwise, and similarly with $\left|f_{i} \wedge v_{k}^{\prime}\right|$.

If there is more than one stack of D-branes on the edge, say $n$ stacks of them, we also must include contributions of $n(n-1) / 2$ massive open strings stretching between the D-branes. As shown in [22] the effect integrating out these strings is

$$
\exp \left(-\sum_{m=1}^{\infty} \frac{1}{m} \operatorname{tr} U_{1}^{m} \operatorname{tr} U_{2}^{m}\right)=\sum_{R}(-1)^{\ell_{R}} \operatorname{Tr}_{R} U_{1} \operatorname{Tr}_{R^{t}} U_{2} .
$$


The relative minus sign in the exponent in (3.17) relative to that of [22] arises as follows. In problem studied in 22] one had two D-branes intersecting on $\mathbf{S}^{1}$ (the $\mathbf{S}^{\mathbf{1}}$ corresponds to the $\mathbf{S}^{\mathbf{1}}$ factor in the D-brane world-volumes which are $L=\mathbf{S}^{\mathbf{1}} \times \mathbf{C}$ ) and the ground state was a boson. Here we have two D-branes whose world-volumes are parallel. There is one normalizable mode of the stretched string supported along an $\mathbf{S}^{\mathbf{1}}$, and it turns out to be a fermion. One way to see this is that, by changing complex structure which does not affect the A-model amplitudes, we can bring the branes to intersect on an $\mathbf{S}^{\mathbf{1}}$ at the expense of turning one D-brane into an anti-D brane. The ground state of the string stretching between them is a fermion, as argued in [21].

For example, for stacks of $m$ D-branes, we have

$$
\sum_{R_{i}, Q_{a, i}^{L}, Q_{a, i}^{R}} C_{R_{j}, R_{k}, R_{i} \otimes_{a=1}^{m} Q_{i, a}^{L}}(-1)^{s(i)} e^{-L(i)} q^{f(i)} C_{R_{i}^{t} \otimes_{a=1}^{m} Q_{i, a}^{R}, R_{j}^{\prime}, R_{k}^{\prime}} \prod_{a=1}^{m} \operatorname{Tr}_{Q_{i, a}^{L}} V_{a} \operatorname{Tr}_{Q_{i, a}^{R}} V_{a}^{-1}
$$

where

$$
\begin{gathered}
L(i)=t_{i} \ell\left(R_{i}\right)+\sum_{a=1}^{m} r_{a} \ell\left(Q_{i, a}^{L}\right)+\left(t_{i}-r_{a}\right) \ell\left(Q_{i, a}^{L}\right) . \\
f(i)=\sum_{a=1}^{m}\left(p_{i} \kappa_{R_{i} \otimes Q_{i, a}^{L}} / 2+\left(p_{i}+n_{i}\right) \kappa_{\left.R_{i}^{t} \otimes Q_{i, a}^{R} / 2\right) .}\right. \\
s(i)=\ell\left(R_{i}\right)+\sum_{a=1}^{m}\left(p_{i} \ell\left(R_{i} \otimes Q_{i, a}^{L}\right)+\left(p_{i}+n_{i}\right) \ell\left(R_{i}^{t} \otimes Q_{i, a}^{R}\right)\right) .
\end{gathered}
$$

\section{Chiral Bosonic Oscillator and the Vertex}

We have seen that the partition functions of the A-model on local toric 3-folds are computable from a set of gluing rules involving a cubic vertex and the propagator. The gluing rules are reminiscent of the construction of the partition function of bosons on a Riemann surface from the "pant diagram" and the tube propagators. We will show that this is not accidental. In fact, as we will argue, the vertex operator and the propagator we have obtained can be viewed as construction of the partition function of the mirror B-model whose geometry, as is well known, is captured by a Riemann surface. Towards this aim in this section we reformulate the vertex and the propagator we have obtained in terms of a free chiral boson on a Riemann surface. In particular, we will show that the winding basis can be identified with the Fock space of the chiral boson. In this connection the sewing rule 
gets mapped identically to the propagator of the chiral boson. Moreover the vertex gets identified with a state in the triple tensor product of the Hilbert space of the free boson. This vertex is highly non-trivial. Even in the classical limit it is more complicated than the usual vertex states one gets for a free boson on a Riemann surface (which is always given by a Bogoliubov transformation and can be represented as exponential of quadratic monomials in the oscillator creation operators).

In the next section we explain how to interpret the free chiral boson as the relevant field for the Kodaira-Spencer theory of gravity [8] in this local context (related ideas have appeared in [23,24]).

\subsection{Reformulation in terms of a chiral boson}

There is a curious similarity between the winding number $\vec{k}$ basis and oscillator states of a free chiral boson. Recall the oscillator expansion of the chiral boson $\phi(u)$,

$$
\partial_{u} \phi(u)=\sum_{m \neq 0} j_{m} e^{m u}
$$

where

$$
\left[j_{m}, j_{n}\right]=m \delta_{m+n, 0}
$$

so that $j_{m>0}$ is the annihilation operator and $j_{m<0}$ the creation operator. The Hilbert space $\mathcal{H}$ of a free chiral boson on a circle is spanned by states of the form

$$
|\vec{k}\rangle=\prod_{m>0} j_{-m}^{k_{m}}|0\rangle
$$

We simply identify the vector $\vec{k}$ above with the vector of winding numbers. With this identification we can interpret the sewing as picking out an element $P$ in the two-fold tensor product of the Hilbert space $P \in \mathcal{H}^{\otimes 2}$, and the vertex as defining a state in the threefold tensor product Hilbert space $C \in \mathcal{H}^{\otimes 3}$. It is natural to ask what these states are.

We first turn to the propagator $P$. We will see that $P$ is the conventional state of the free chiral boson on a cylinder. The path integral of a free chiral boson on a cylinder of length $t$ is a state in the tensor product Hilbert space $P \in \mathcal{H}_{1} \otimes \mathcal{H}_{2}$ (where $\mathcal{H}_{1,2}$ are associated to the two boundaries) given by

$$
|P\rangle=\exp \left(-t \sum_{m>0} \frac{1}{m} j_{-m}^{1} j_{m}^{2}\right)|0\rangle_{1} \otimes\left\langle\left. 0\right|_{2} .\right.
$$


Expanding the exponential we get

where

$$
\sum_{\vec{k}} e^{-\ell(\vec{k}) t} \frac{(-1)^{h}}{z_{\vec{k}}}|\vec{k}\rangle \otimes\langle\vec{k}|
$$

$$
z_{\vec{k}}=\prod_{m} k_{m} ! m^{k_{m}} .
$$

This is precisely the gluing rule we had discussed for A-model amplitudes!

We now turn to the vertex $C$. As we said above, the vertex amplitude, as formulated in section 3 ,

$$
Z=\sum_{\vec{k}_{i}} C_{\vec{k}_{1} \vec{k}_{2} \vec{k}_{3}} \frac{1}{\prod_{i} z_{k_{i}}} \operatorname{Tr}_{\vec{k}_{1}} V_{1} \operatorname{Tr}_{\vec{k}_{2}} V_{2} \operatorname{Tr}_{\vec{k}_{3}} V_{3}
$$

can be written in terms of the state $C$ in $\mathcal{H}^{3}$ of the chiral boson as

$$
Z=\sum_{\vec{k}_{i}} \operatorname{Tr}_{\vec{k}} V_{1} \operatorname{Tr}_{\vec{k}} V_{2} \operatorname{Tr}_{\vec{k}} V_{3} \frac{1}{\prod_{i} z_{k_{i}}}\left\langle\vec{k}_{1}\right| \otimes\left\langle\vec{k}_{2}\right| \otimes\left\langle\vec{k}_{3} \mid C\right\rangle
$$

Note that, at the level of the answer, $|C\rangle$ is given by

$$
|C\rangle=\exp \left(\sum_{\vec{k}_{i}} F_{\vec{k}_{1}, \vec{k}_{2}, \vec{k}_{3}}\left(g_{s}\right) j_{-\vec{k}_{1}} j_{-\vec{k}_{2}} j_{-\vec{k}_{3}}\right)|0\rangle_{1} \otimes|0\rangle_{2} \otimes|0\rangle_{3} .
$$

where $F$ is identified with the free energy of the topological string, and $j_{ \pm \vec{k}}=\prod_{m>0} j_{ \pm m}^{k_{m}}$. since "evaluating" the amplitude, i.e. performing contractions, amounts to replacing the creation operators in the free energy with $V^{\prime} s, t r V^{m} \leftrightarrow j_{-m}$. It is natural to ask what the meaning of the three point vertex $C \in \mathcal{H}^{\otimes 3}$ is. One may at first think that this may be related to that of a free boson on trice punctured sphere. This is almost true. Namely it is a state associated with a sphere with three punctures, but the theory is that of a free scalar theory only to leading order in oscillator expansion and in the $g_{s} \rightarrow 0$ limit. We will discuss this and its interpretation after we discuss the B-model interpretation of the chiral boson as describing the quantum field of the Kodaira-Spencer theory on the Riemann surface. We will explain why there are more oscillator terms in $C$, including the existence of non-trivial $g_{s}$ corrections. A full study of the vertex $C$ from the B-model perspective will be done in [25].

For now, note that, most remarkably, the D-branes can be thought of as coherent states in the chiral boson theory! The vertex amplitude $Z$ is computed by inserting

$$
\langle V|=\langle 0| \exp \left(\sum_{m>0} \frac{1}{m} \operatorname{tr} V^{m} j_{m}\right)
$$

at each of the three punctures that give rise to the vertex state $C$,

$$
Z=\left\langle V_{1}\right| \otimes\left\langle V_{2}\right| \otimes\left\langle V_{3} \mid C\right\rangle .
$$

We will explain this below. 


\section{Local B-model mirror and the quantum Kodaira-Spencer theory}

We have seen that the vertex is naturally captured by the states of a chiral boson on a sphere with three punctures. In this section we will identify the chiral boson on each patch as the quantum field of the Kodaira-Spencer theory on the mirror B-model involving a Riemann surface. The modes of the chiral boson are affected, as we will explain, by the degrees of freedom $\left(\operatorname{tr} V^{n}\right)$ on the B-branes. In particular we will explain, from the Bmodel perspective, why the open string amplitudes know about the closed string B-model amplitudes. Moreover we identify the branes in this setup as the fermions associated to the chiral bosons $\psi(z)=e^{\phi(z)}$. We use this picture to compute leading terms in the oscillator expansion of the vertex. Extension to the full vertex, from this perspective will appear elsewhere [25]. Moreover the gluing rules of the vertex can now be directly interpreted as computations of the Kodaira-Spencer theory in the operator formulation on the mirror Riemann surface.

The target space of B-model was interpreted in [8] as describing the quantum theory of complex deformation of the Calabi-Yau threefold. This in particular applies to the local Calabi-Yau case at hand. In particular, if we consider the A-model in the local toric case, as already discussed in section 2 , the mirror is given by a hypersurface in $(x, \tilde{x}, u, v) \in \mathbf{C} \times \mathbf{C} \times \mathbf{C}^{*} \times \mathbf{C}^{*}:$

$$
x \tilde{x}=F(u, v)
$$

Moreover $F(u, v)$ can be obtained by gluing pant diagrams of the form

$$
e^{u}+e^{v}+1=0 .
$$

The holomorphic 3 -form is given by

$$
\Omega=d x d u d v / x
$$

As is well known in the local context, integration of $\Omega$ over the non-trivial class of three cycles gets reduced to computation of a 1-form on the Riemann surface. The only nontrivial 3-cycles are formed by the $\mathbf{S}^{\mathbf{1}}$ fibration (identified with $\left.(\tilde{x}, x) \rightarrow\left(e^{i \theta} \tilde{x}, e^{-i \theta} x\right)\right)$ over a domain in the $u, v$ plane bounded by the Riemann surface $(\Sigma: F(u, v)=0)$. The integral of $\Omega$ over such cycles reduces to integrals of the meromorphic 1-form

$$
\lambda=u d v
$$


on the 1-cycles of the non-compact Riemann surface $\Sigma$. Note that $\lambda$ is not globally well defined, and it makes sense only patch by patch. In particular if we had considered the $u$-patch, integration of the 2-cycle fiber would have resulted in a 1-form $-v d u$. It is this lack of global definition of $\lambda$ which lead to non-trivial interactions, to an otherwise free theory.

The variations of the complex structure and the corresponding period integral get mapped to the variation of the complex structure of $\Sigma$ and the periods of the corresponding reduced 1 -form $\lambda$ on it. Note that there is a direct relation between changing the complex structure of $\Sigma$ and the choice of $\lambda$. In particular suppose we are in the $v$-patch defined by being centered at $e^{v}=0$; Consider the complex deformation

$$
F \rightarrow F(u, v)+\delta F(u, v)
$$

The above derivation for the reduced one form will still go through without any change,

$$
\lambda=u d v
$$

but now $u$ is a different function of $v$.

Solving $F(u, v)=0$ we would in principle get $u=f(v)$ and under the complex deformation we have

$$
u=f(v)+\delta f
$$

and so the change in the 1 -form $\lambda$ is given by

$$
\delta \lambda=\delta f d v
$$

Thus, as in [8] the basic quantum field gets identified with this variation. For this to be a good deformation of complex structure $\delta f$ should be a meromorphic function of $e^{v}$, i.e.

$$
\bar{\partial}_{v} \delta f=0
$$

To get an ordinary quantum field it is natural to write $\delta f=\partial \phi_{v}$ in which case (5.2) gets mapped to

$$
\bar{\partial} \partial \phi_{v}=0
$$

In terms of this scalar the variation of the 1 -form is given by

$$
\delta \lambda=\partial \phi_{v}
$$


and the equation (5.3) is sufficient for the condition of integrability of the complex structure, unlike the generic 3-fold complex structure deformation where the story is more complicated. Thus we have a free boson propagating on each patch.

In the classical theory we can of course parameterize the deformation in any way we want, however for the quantum theory writing the variation this way is more natural. This is because the Kodaira-Spencer theory in the formulation of [8] has the kinetic term of the form

$$
\frac{1}{g_{s}^{2}} \int_{\text {Calabi Yau }} \omega \partial^{-1} \bar{\partial} \omega
$$

where $\omega$ is a $(2,1)$-form representing the change in the complex structure of Calabi-Yau. It is natural to write, at least for a patch, $\omega=\partial \chi$ where $\chi$ is a $(1,1)$ form. In terms of $\chi$ the action would become

$$
\frac{1}{g_{s}^{2}} \int_{\text {Calabi Yau }} \partial \chi \bar{\partial} \chi
$$

In the local context that we are discussing, $\chi$ gets identified with the $\phi_{v}$ above which is a scalar on $\Sigma$, and we get the free scalar theory

$$
\frac{1}{g_{s}^{2}} \int_{\Sigma} \partial \phi \bar{\partial} \phi .
$$

Note that the anti-holomorphic piece of $\phi$ is a gauge artifact: Shifting $\phi$ by an antiholomorphic function will not affect $\partial \phi$ and so does not change the 1-form $\lambda$. So $\phi$ should be viewed as a chiral boson. We can study the Kodaira-Spencer theory patch by patch by chiral fields $\phi_{v}$. We will write the variation of the complex structure as

$$
u=f_{0}(v)+\partial_{v} \phi_{v}
$$

We can also absorb $f_{0}(v)$ as a classical vev for $\partial_{v} \phi_{v}$, which we will sometimes do. In the $v$-patch which is cylindrical we can write

$$
\partial_{v} \phi_{v}=\sum_{n>0} j_{n} e^{-n v}+g_{s}^{2} j_{-n} e^{n v}
$$

where we have included factors of $g_{s}^{2}$ to account for the kinetic term of the scalar being $1 / g_{s}^{2}$. In the quantum formulation $j_{-n}$ and $j_{n}$ are not independent, and correspond to creation and annihilation operators. To better understand this we will consider a coherent set of states given by replacing

$$
j_{n} \rightarrow t_{n}
$$


This is natural in this patch, because changing the complex structure at the infinity of this patch, corresponds to changing $\partial_{v} \phi_{v}$ at $e^{v} \rightarrow 0$, and that is determined by the negative powers $e^{-n v}$ above. However now the positive powers of $e^{n v}$ are determined, quantum mechanically. Let $\langle\Sigma|$ denote the state created by the rest of the Riemann surface. Let the coherent states be defined by

$$
|t\rangle=\exp \left(\sum_{n>0} \frac{1}{n} j_{-n} t_{n}\right)|0\rangle
$$

Let us denote the partition function of the theory including the $t_{n}$ deformations by $Z(t)$. Then we have

$$
Z(t)=\exp \left(F\left(g_{s}, t_{n}\right)\right)=\langle\Sigma \mid t\rangle
$$

To justify constructing the coherent state in terms of $j_{-m}$ alone, note that if we consider the expectation value of $\partial_{v} \phi_{v}$ on the cylinder at infinity where $e^{-v} \rightarrow \infty$ then $e^{-n v}$ terms in (5.5) dominate. This can also be viewed as changing the 1 -form $\lambda$ at infinity by

$$
\delta \lambda=d v \sum_{n} t_{n} e^{-n v}
$$

On the other hand if we consider the expectation value of $\partial_{v} \phi_{v}$ for the positive powers $e^{n v}$ it will not be zero. It might at first appear that in the classical limit $g_{s} \rightarrow 0$ this would be zero because of the explicit $g_{s}^{2}$ dependence in (5.5), but this is not the case. This is because $F\left(g_{s}, t_{n}\right)$ has a $1 / g_{s}^{2}$ term in the genus zero part given by $F_{0}\left(t_{n}\right)$, so this survives in the limit. This will give us for the expectation value of $\partial_{v} \phi_{v}$ in the limit $g_{s} \rightarrow 0$

$$
\frac{1}{Z}\left\langle\Sigma\left|\partial_{v} \phi_{v}\right| t\right\rangle=\sum_{n>0} t_{n} e^{-n v}+n \frac{\partial F_{0}}{\partial t_{n}} e^{n v} .
$$

which means that classically we have

$$
\partial_{v} \phi_{v}=\sum_{n>0} t_{n} e^{-n v}+n \frac{\partial F_{0}}{\partial t_{n}} e^{n v} .
$$

Here we need to clarify one important point: $F$ is not an unambiguous function of $t_{n}$ 's. This depends on how we choose the coordinates on each patch. Changing the coordinates, will give rise to a different function $F\left(t_{i}\right)$. The difference between these results is the same as the Virasoro action. This dependence on the choice of the local coordinates on the Riemann surface will turn out to be related to the framing ambiguity. For us the Riemann surface comes with almost canonical coordinates involving combinations of $u$ and $v$ with 
$d u \wedge d v$ making sense in the full Calabi-Yau. In a given $v$-patch we will have the situation where $z=e^{v} \rightarrow 0$, i.e. $v \rightarrow-\infty$ is on the patch, and $u \rightarrow$ const. as $z \rightarrow 0$. This almost uniquely fixes the coordinates except for an integer choice: The $d u \wedge d v$ is invariant under $S L(2, \mathbf{Z})$. There is a subset of $S L(2, \mathbf{Z})$, indexed by an integer $n$, which preserve the conditions we have put on each patch, namely $u^{\prime}=u$ and $v^{\prime}=v+n u$. Note that the coefficient of $v$ in $v^{\prime}$ is 1 because we want $z^{\prime}=O(z)$ as $z \rightarrow 0$ in order to have a good coordinate. This transformation will give a new one form $\lambda=u^{\prime} d v^{\prime}$ and a new coordinate

$$
z^{\prime}=e^{v^{\prime}}=e^{n u} z
$$

where $e^{u}=\sum_{i=0}^{\infty} a_{i} z^{i}$. For example consider the pant Riemann surface

$$
e^{-u}+e^{v}+1=0
$$

in the $v$-patch, which includes $v \rightarrow-\infty$ (where $u \rightarrow i \pi$ ). If we now change coordinates $v^{\prime}=v+n u$ we will have

$$
z^{\prime}=z\left[(-1)^{n}(1+z)^{-n}\right]
$$

So if we compute $F$ in the $z$ patch, in the new coordinate patch we will need to exponentiate an appropriate element of Virasoro algebra which changes the coordinates. Thus the choices of $F$ is indexed by an integer $n$ in each patch.

In the interest of comparison with our A-model vertex we will now specialize to the case of the pant diagram, which is mirror to $\mathbf{C}^{\mathbf{3}}$, given by the Riemann surface

$$
e^{u_{1}}+e^{u_{2}}+e^{u_{3}}=0
$$

where one variable is eliminated by rescaling the equation. This way of writing it, exhibits the cyclic symmetry between the three patches. A choice of coordinates that corresponds to the "standard framing of the vertex" discussed before and which preserves the $\mathbf{Z}_{3}$ symmetry is as to let $u=u_{3}-u_{1}$ and $v=u_{2}-u_{3}$, and $w=u_{1}-u_{2}$ then we have

$$
\begin{array}{lll}
e^{-u}+e^{v}+1=0 & \lambda=v d u & u \text {-patch } \\
e^{-v}+e^{w}+1=0 & \lambda=w d v & v \text {-patch } \\
e^{-w}+e^{u}+1=0 & \lambda=u d w & w-\text { patch }
\end{array}
$$

Note that we also have the relation

$$
u+v+w=0
$$


Changing of the coordinates by framings $\left(n_{1}, n_{2}, n_{3}\right)$ is obtained by the choice of the coordinates

$$
\begin{gathered}
u \rightarrow u+n_{1} v \\
v \rightarrow v+n_{2} w \\
w \rightarrow w+n_{3} u
\end{gathered}
$$

In each patch we can deform the defining equation by a chiral scalar, as discussed above. The corresponding scalars we call $\phi_{u}, \phi_{v}, \phi_{w}$. The equation of the surface gets modified, when $\phi_{i} \neq 0$ by following the deformation discussed in general above, and we get

$$
\begin{gathered}
e^{-u}+e^{v+\partial_{u} \phi_{u}}+1=0 \\
e^{-v}+e^{w+\partial_{v} \phi_{v}}+1=0 \\
e^{-w}+e^{u+\partial_{w} \phi_{w}}+1=0
\end{gathered}
$$

where in the classical limit

$$
\begin{aligned}
\partial_{u} \phi_{u} & =\sum_{n>0} t_{n}^{u} e^{-n u}+n \frac{\partial F_{0}}{\partial t_{n}^{u}} e^{n u} . \\
\partial_{v} \phi_{v} & =\sum_{n>0} t_{n}^{v} e^{-n v}+n \frac{\partial F_{0}}{\partial t_{n}^{v}} e^{n v} . \\
\partial_{w} \phi_{w} & =\sum_{n>0} t_{n}^{w} e^{-n w}+n \frac{\partial F_{0}}{\partial t_{n}^{w}} e^{n w} .
\end{aligned}
$$

If we assume $\partial \phi$ 's are smalla, we get the following equations:

$$
\begin{aligned}
& e^{-u}+e^{v}+1+e^{v} \partial_{u} \phi_{u}=0 \\
& e^{-v}+e^{w}+1+e^{w} \partial_{v} \phi_{v}=0 \\
& e^{-w}+e^{u}+1+e^{u} \partial_{w} \phi_{w}=0
\end{aligned}
$$

If we multiply equation $(i)$ by $e^{-v}$, and use the fact that $u+v=-w$ we get

$$
e^{w}+1+e^{-v}+\partial_{u} \phi_{u}=0
$$

4 This derivation of the small $\partial \phi$ limit of the vertex was suggested to us by Robbert Dijkgraaf. 
Comparing this with equation $(i i)$ we learn that

$$
\partial_{u} \phi_{u}=e^{w} \partial_{v} \phi_{v}
$$

On the other hand, to leading order we have from equation (i),

$$
d v / d u=e^{-u-v}=e^{w}
$$

we thus have

$$
\partial_{u} \phi_{u}=\frac{d v}{d u} \partial_{v} \phi_{v}
$$

and by the $\mathbf{Z}_{\mathbf{3}}$ cyclic symmetry similar equations with $u \rightarrow v \rightarrow w \rightarrow u$. This in particular implies that in the classical limit, and to leading order in oscillators the three $\phi$ 's in the three patches can be viewed as coming from a global $\phi$. This means that to leading order in the classical limit and in oscillators, the vertex operator should be the standard one coming from the well known techniques of operator formulation on Riemann surfaces [26, 27,28] which lead to Bogoliubov transformations.

Here we will digress to review this derivation.

\subsection{Bogoliubov transformation}

Consider a chiral boson on a Riemann surface mirror to $\mathbf{C}^{\mathbf{3}}$. The Riemann surface is a sphere with three punctures, and the path integral on this gives a state in the tensor product of three free Hilbert spaces $\mathcal{H}_{1} \otimes \mathcal{H}_{2} \otimes \mathcal{H}_{3}$ corresponding to the punctures. Moreover, mirror symmetry provides us with a choice of complex structure on the punctured Riemann surface, and this gives a canonical choice of coordinates $z_{i}=e^{u_{i}}$ near each puncture at $z_{i}=0$ and transition functions relating them. The transition functions between the patches give rise to Bogoliubov transformations that relate the three Hilbert spaces and these are sufficient to determine the ray in the Hilbert space to which the path integral corresponds to. To do so we follow [27]. Note that the path integral of the chiral boson $\phi$ has infinite dimensional group of symmetries corresponding to shifting $\phi \rightarrow \phi+f$ for any function $f$

which is holomorphic on the punctured Riemann surface (a meromorphic function whose only poles are at the punctures). This gives rise to conserved charge

$$
Q(f)=\sum_{i} \oint_{z_{i}=0} f\left(z_{i}\right) \partial \phi,
$$


which must annihilate the path integral. In each of the three patches we have a different expansion for the chiral boson in terms of the local holomorphic coordinate.

$$
\phi\left(z_{i}\right)=\sum_{m \neq 0} \frac{1}{m} j_{-m}^{(i)} z_{i}^{m}
$$

The three patches are related by (5.6), where we put $z_{1}=e^{u}, z_{2}=e^{v}$ and $z_{3}=e^{w}$, so that the three patches are related by

$$
z_{i+1}+\frac{1}{z_{i}}+1=0
$$

Then, for example, a meromorphic function $f_{m}^{(1)}=z_{1}^{m}$ has expansion

$$
z_{1}^{m}=\sum_{n \geq m} \frac{m}{n} O_{n, m} z_{2}^{-n}=\sum_{m \geq n} O_{m, n} z_{3}^{-n}
$$

where

$$
O_{m, n}=(-1)^{m}\left(\begin{array}{c}
m \\
n
\end{array}\right)
$$

The corresponding charge $Q(f)$ is given by

$$
Q\left(f_{m}^{(1)}\right)=j_{m}^{(1)}+\sum_{n \geq m} O_{m, n} j_{-n}^{(2)}+\sum_{n \geq m} \frac{m}{n} O_{n, m} j_{-n}^{(3)}
$$

The conditions that $Q\left(f_{m}^{(i)}\right)$ annihilate the path integral suffice to determine it:

$$
|Z\rangle=\exp \left(\sum_{m>0, n \geq m} \frac{O_{n, m}}{n}\left[j_{-m}^{(1)} j_{-n}^{(2)}+j_{-m}^{(2)} j_{-n}^{(3)}+j_{-m}^{(3)} j_{-n}^{(1)}\right]\right)|0\rangle_{1} \otimes|0\rangle_{2} \otimes|0\rangle_{3} .
$$

Here we have suppressed the linear term in the $j_{-m}$ 's corresponding to the fact that the vacuum $|0\rangle$ is not the ordinary vacuum, but $\partial \phi$ has a piece corresponding to the classical geometry, which we have been shifting away so far. Let us now restore it. The classical piece of the chiral boson in the u-patch, for example, is $\partial \phi(u)_{0}=v(u) d u=\log \left(1+e^{-u}\right) d u$. Shifting this away in the action $\frac{1}{g_{s}^{2}} \int \partial \phi \bar{\partial} \phi$ gives a surface term

$$
\frac{1}{g_{s}} \oint \phi_{0} \partial \phi=-\sum_{n>0} \frac{(-1)^{n}}{n^{2}} j_{-n}^{(1)}
$$

(after rescaling $\phi \rightarrow g_{s} \phi$ ), so this shifts the vacuum $|0\rangle_{1}$ to

$$
|0\rangle_{1} \rightarrow \exp \left(-\sum_{n>0} \frac{(-1)^{-n}}{n^{2} g_{s}} j_{-n}^{(1)}\right)|0\rangle_{1},
$$


and similarly in the other two patches.

The state we have computed should be accurate to leading order in $g_{s}$ and up to quadratic terms in $j_{-m}=t_{m}$. The full vertex will have additional terms both in the $g_{s}$ corrections as well as in terms involving more $t_{m}$ 's. This is because the derivation leading to a global chiral boson was valid only in this limit. A full discussion of this from the B-model perspective will be presented elsewhere [25].

From the B-model perspective if we know what the path-integral gives for the pantdiagram, then we can obtain any other amplitude by gluing. This is because the KodairaSpencer equation implies that in each patch $\phi$ is a chiral boson with the standard propagator. However we have to make sure that in the gluing the coordinate choices match-this is the same as making sure that the framings are compatible in the A-model computation of the vertex. Independently of how one computes this trivalent vertex, the knowledge of

$$
F=\sum_{g} F_{g}\left(t_{n}^{u}, t_{n}^{v}, t_{n}^{w}\right)\left(g_{s}\right)^{2 g-2}
$$

will capture arbitrary B-model local amplitudes, as everything can be obtained from this by gluing, as discussed in section 2. We thus appear to have a system involving closed strings on the pant-diagram, in the operator formalism, capturing arbitrary local models. On the other hand we have given, motivated from the A-model considerations a similar

gluing rule and a vertex $C$ involving amplitudes of the A-branes on $\mathbf{C}^{3}$, which is mirror to the pant diagram. To complete the circle of ideas we have to connect these two facts. The mirror of A-branes are B-branes, which on the Riemann surface get mapped to points on the pant diagram [19]. So the question, posed in purely B-model context, is the following: What is the relation of B-branes with closed string Kodaira-Spencer amplitudes? We will explain how this works in the next section.

\subsection{B-branes and closed string B-model}

We will now argue how $F$ defined in (5.11) in terms of operator formulation of closed string target theory, i.e. the quantum Kodaira-Spencer theory, can be equivalently phrased in terms of open string B-model amplitudes. To this end we will have to understand the effect of B-brane on closed string B-model. We will show that the insertion of a B-brane at the point $z$ is equivalent, for the reduced Kodaira-Spencer theory, to the insertion of the fermionic field $\psi(z)=e^{\phi}$. In other words, fermions, which are usually viewed as soliton of 
a chiral scalar can also be viewed as the B-branes, i.e., the soliton of the Kodaira-Spencer theory.

Consider B-model in the local patch given by $v$ where $e^{v} \rightarrow 0$ is part of the patch. Consider placing many branes at the circumference of the cylinder given by $v=v_{i} \ll 0$. We ask how putting B-branes back reacts on the gravity B-model? In other words, how does the complex structure get modified by the B-branes? To answer this question we consider adding an extra brane, viewing it as a probe, and place it at $v$ in this patch. We are interested in what the effect of many branes are on the probe. If the probe is placed at $v \ll 0$ then the effect is simply given by summing over all the open strings stretching between them. This is the mirror of the computation of [22] and was discussed in the mirror setup in 29]. The effect on the free energy of the probe at $v$ upon integrating out the stretched strings between branes at $v_{i}$ and the probe is

$$
\delta F=\partial \phi=\sum_{i, n>0} \frac{1}{n} e^{-n\left(v-v_{i}\right)}=\sum_{n>0} \frac{1}{n} t r V^{n} e^{-n v}
$$

where we have used the fact that $e^{v_{i}}$ are the eigenvalues of $V$. Note that for $v \ll 0$ there is no contribution from the rest of the surface to the free energy of the probe. On the other hand we can ask which deformed geometry will give rise to this free energy probed by the brane. This is given by the computation in [19] where

$$
g_{s} F=\int^{v} \lambda=\int^{v} \partial_{v} \phi=\phi(v)+\text { const. }
$$

where $\lambda$ is the deformed 1 -form on the surface and we have absorbed back into $\phi$ the classical piece of the 1 -form. Thus placing a brane at $v$ affects the Kodaira-Spencer action by the addition of $\phi(v)$. Let us consider how $F$ changes for $v \ll 0$. Let us call this singular part of $\phi$ by $\phi^{-}$. In particular the deformed one form $\lambda^{-}$which dominates for $v \ll 0$ gets identified with

$$
\phi^{-}(v)=g_{s} F
$$

We thus see that the free energy felt by the probe brane (5.12) is reproduced by the deformation

$$
\phi^{-}(v)=\sum_{n>0} \frac{1}{n} t r V^{n} e^{-n v}
$$

which leads to the identification

$$
t_{n}=\operatorname{tr} V^{n}
$$


This explains the observation we made before about the role of the chiral boson in the vertex we had obtained from A-model considerations.

This suggests the following interpretation: To have a brane at the point $v$ we add to the KS action the operator $\phi(v) / g_{s}$, or to the path integral the operator

$$
\exp \left(\phi(v) / g_{s}\right)
$$

(the $1 / g_{s}$ there is to remind us that the disk amplitude is proportional to $1 / g_{s}$ ). This leads to the same response in the free energy. We can redefine $\phi / g_{s} \rightarrow \phi$, which gets rid of the $1 / g_{s}^{2}$ in the kinetic term. We are thus led to identify the operator inserting the brane at $z$ with the insertion in the path-integral of

$$
\psi(z)=e^{\phi(z)}
$$

i.e. the fermion operator! (we have absorbed an $i$ in the definition of $\phi$ in comparison with the conventional description of bosonization). Connection between fermions and the D-branes was anticipated a while back [30] and this result makes this concrete. The antibranes get identified with

$$
\psi^{\dagger}(z)=e^{-\phi(z)}
$$

This is because the free energy will change sign for an anti-brane. As a check of this statement, note that the coherent state involving the branes at $v_{i}$ can be viewed as the state

$$
\prod_{i} \exp \left(\phi\left(v_{i}\right)\right)|0\rangle=\exp \left(\sum_{i, n>0} \frac{1}{n} e^{n v_{i}} j_{-n}\right)|0\rangle=\left|t_{i}\right\rangle
$$

with $t_{n}=\sum_{i} \exp \left(n v_{i}\right)$. Note that in this expression we have normal ordered the operator. Not normal ordering it would have also led to the effect of the branes on each other; i.e. the result of integrating out the open string stretched between them. We will now present an additional argument why the fermion field is the B-brane operator.

Consider a general Calabi-Yau threefold. Consider wrapping $N$ B-branes over a (compact or non-compact) holomorphic curve $C$ in Calabi-Yau. This curve is of real codimension 4 in the Calabi-Yau and is surrounded by a 3 -cycle $F_{C}$. Consider the integral

$$
I_{N}=\int_{F_{C}} \Omega_{N}
$$


where $\Omega_{N}$ is the holomorphic 3 -form, corrected by the fact that there are $N$ B-branes on $C$. Then we claim

$$
I_{N}-I_{0}=N g_{s}
$$

This is related to the mirror of the Chern-Simons/topological strings duality of [2]. For example consider $N$ B-branes wrapping the $\mathbf{P}^{1}$ in $\mathrm{O}(-1)+\mathrm{O}(-1) \rightarrow \mathbf{P}^{1}$. Then there is an $\mathbf{S}^{\mathbf{3}}$ surrounding $\mathbf{P}^{1}$ and the claim is that

$$
\int_{S^{3}} \Omega_{N}=N g_{s}
$$

In particular under the large $N$ duality this is consistent with the size of the $\mathbf{S}_{\mathbf{3}}$ being given by $N g_{s}$. This statement should hold for compact or non-compact branes as it is a local question and it is our definition of the B-brane in terms of its coupling to the gravitational Kodaira-Spencer theory.

In the context of the local model we are considering here, the B-brane wraps over a non-compact plane, which intersects the Riemann surface at a point. The integral of the holomorphic three-form around it, reduces to the integral of the 1-form $\lambda$ around this intersection point. So if we denote by $B(z)$ the field creating a D-brane at point $z$ on the Riemann surface, then

$$
\oint_{z} \lambda\left(z^{\prime}\right) B(z)=g_{s} \oint_{z} \partial_{z^{\prime}} \phi\left(z^{\prime}\right) B(z) d z^{\prime}=g_{s} .
$$

This is indeed the correct OPE defining the fermionic field and we get the identification

$$
B(z)=\psi(z)=e^{\phi(z)}
$$

Thus the trivalent topological vertex can be viewed as computing

$$
\left\langle\prod_{i} \psi\left(u_{i}\right) \prod_{j} \psi\left(v_{j}\right) \prod_{k} \psi\left(w_{k}\right)\right\rangle
$$

in the closed string mirror formulation of the problem. It is then clear why the amplitudes involving brane can lead to a full reconstruction of the closed string amplitudes: This is simply the familiar bosonization! We can also consider mixed amplitudes with some branes left over in a closed string background. In the B-model all we need to do is to add certain fermion operators. Note that the framing ambiguity for the B-branes gets mapped to the fact the $\psi(z)$ is a half-differential and so the amplitudes will depend on the coordinates chosen. 


\section{The derivation of the vertex amplitude}

In this section we will provide a derivation of the three-point vertex in the A-model. It turns out to be more convenient to switch back to the representation basis in deriving the cubic vertex $C_{R_{1}, R_{2}, R_{3}}$. We will derive this vertex using the large $N$ topological duality [2] relating large $N$ Chern-Simons amplitudes with those of closed topological strings. We will be able to compute the full vertex to all orders in $g_{s}$ and for arbitrary oscillator numbers. This will allow us to confirm, in section 8, that this agrees, in the $g_{s} \rightarrow 0$ limit, with the linear and quadratic pieces of oscillators which we have computed from the perspective of the B-model in the previous section.

As was conjectured in [2] and proven in [31], the topological open string A-model of $N$ D-branes on $\mathbf{S}^{3}$ in $Y=T^{*} \mathbf{S}^{3}$ is the same as the topological A-model closed string theory on $X=\mathcal{O}(-1) \oplus \mathcal{O}(-1) \rightarrow \mathbf{P}^{1}$. The large $N$ duality is a geometric transition where the $\mathbf{S}^{3}$ and the D-branes disappear and get replaced by the $\mathbf{P}^{\mathbf{1}}[2]$. The string coupling constant is the same in both theories, and the size $t$ of the $\mathbf{P}^{\mathbf{1}}$ is identified with the 't Hooft coupling $t=N g_{s}$. The open string theory, as was shown in [32], is the same as $U(N)$ Chern-Simons theory on $\mathbf{S}^{3}$ where the level $k$ of the Chern-Simons is related to the string coupling as $g_{s}=\frac{2 \pi i}{k+N}$. Various aspects of this duality have been studied in [22,33, 34, 35, in particular in 22] the duality was studied in the presence of non-compact D-branes. A variant of this is what we need here.

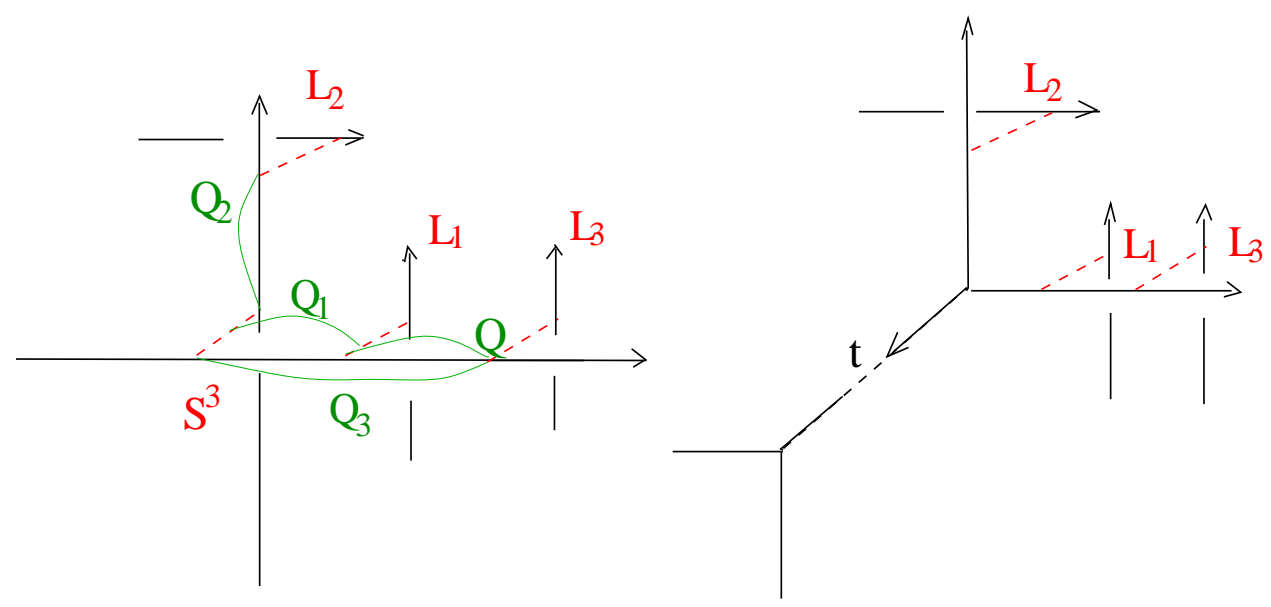

Fig. 9 The figure on the left corresponds to Chern-Simons theory on $\mathbf{S}^{3}$ with three source D-branes. $Q_{1,2,3}, Q$ denote the bifundamental strings. In the large $N$ limit, the $\mathbf{S}^{3}$ undergoes a geometric transition. The figure on the right depicts the large $N$ dual geometry, with Lagrangians $L_{1,2,3}$, after the transition. The local patch where the D-branes are is a $\mathbf{C}^{\mathbf{3}}$. 
Consider then $T^{*} \mathbf{S}^{3}$ with $N$ D-branes on the $\mathbf{S}^{3}$, but in addition $N_{2}$ D-branes on one leg wrapping the Lagrangian $L_{2}$, and $N_{1}$ and $N_{3}$ D-branes on the other, wrapping $L_{1}$ and $L_{3}$ respectively, as depicted in fig. 9. In the dual theory we end up with $Y=$ $\mathcal{O}(-1) \oplus \mathcal{O}(-1) \rightarrow \mathbf{P}^{\mathbf{1}}$. The D-branes wrapping the compact cycle have disappeared, but the D-branes on the non-compact cycles are pushed through the transition. The resulting configuration is shown in the second picture in fig. 9. The amplitude corresponding to D-branes on $Y$ can easily be calculated using solvability of Chern-Simons theory and the large $N$ transition. In the limit where $N \rightarrow \infty$, the size of the $\mathbf{P}^{\mathbf{1}}$ in $Y$ grows, and zooming in on the vertex with the D-branes, we are left with $\mathbf{C}^{3}$ and the three D-branes. This is not exactly the configuration of D-branes that gives the three-point vertex, but it turns out to be close enough; we need to move the $L_{1}$ Lagrangian D-branes through the vertex and put it on the other leg of $\mathbf{C}^{3}$. We will explain below how this can be achieved.

The open string theory on the $\mathbf{S}^{3}$ is $U(N)$ Chern-Simons theory with some matter fields coming from the three non-compact Lagrangians $L_{1,2,3}$. As shown in [3], there are bifundamental strings stretching between the $\mathbf{S}^{\mathbf{3}}$ and $L_{1,2,3}$, and is in addition there are strings between $L_{1}$ and $L_{3}$. The ground state of all of these strings in the topological A-model is a bifundamental matter field, and integrating it out corresponds to inserting an annulus operator (3.17).

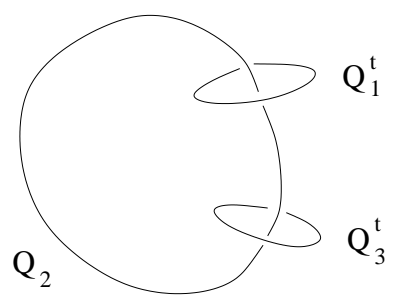

Fig. 10 This is a three-component link which is obtained from the Hopf link by "doubling" one of its components, i.e. by replacing it with two unlinked unknots. The labels denote representations, and the Chern-Simons invariant associated to the link is $S_{\bar{Q}_{2} Q_{1}^{t} \otimes Q_{2}^{t}} / S_{00}$.

Keeping track of the orientations, we have that

$$
\begin{aligned}
Z\left(V_{1}, V_{2}, V_{3}\right)=\frac{1}{S_{00}} \sum_{Q_{1}, Q_{2}, Q_{3}, Q}(-1)^{\ell\left(Q_{1}\right)}\left\langle\operatorname{Tr}_{Q_{2}} U \operatorname{Tr}_{Q_{1}^{t} \otimes Q_{3}^{t}} U\right\rangle \\
\times \operatorname{Tr}_{Q_{1}} V_{1} \operatorname{Tr}_{Q^{t}} V_{1}^{-1} \operatorname{Tr}_{Q_{2}} V_{2} \operatorname{Tr}_{Q_{Q} \otimes Q_{3}} V_{3} .
\end{aligned}
$$


where we have put the $1 / S_{00}$ in front to compute the contribution to the partition function due to the branes, noting that $S_{00}$ is the partition function of topological string on $\mathcal{O}(-1) \oplus$ $\mathcal{O}(-1) \rightarrow \mathbf{P}^{\mathbf{1}}$. In the equation above the $V_{i}$ is related to the holonomy on the $i-$ th stack of non-compact D-branes and $U$ is holonomy on the $\mathbf{S}^{3}$. The vacuum expectation value in (6.1) corresponds to a Hopf link with one of its components replaced by two unlinked unknots, as in fig. 10, and evaluated on the $\mathbf{S}^{3}$. The unnormalized expectation value is given by 18

$$
\left\langle\operatorname{Tr}_{Q_{2}} U \operatorname{Tr}_{Q_{1}^{t} \otimes Q_{3}^{t}} U\right\rangle=S_{Q_{1}^{t} \otimes Q_{3}^{t} \bar{Q}_{2}},
$$

where $S$ is the S-matrix of the $U(N)_{k}$ WZW model. On the other hand, it is well known that $S_{Q_{i} \otimes Q_{j} \bar{Q}_{l}}=S_{Q_{i} \bar{Q}_{l}} S_{Q_{j} \bar{Q}_{l}} / S_{0 \bar{Q}_{l}}$ [36,18]. Using this, we arrive at the following expression for $(6.1)$ :

$$
Z\left(V_{1}, V_{2}, V_{3}\right)=\sum_{Q_{1}, Q_{2}, Q_{3}, Q}(-1)^{\ell\left(Q_{1}\right)} \frac{S_{Q_{1}^{t} \bar{Q}_{2}} S_{Q_{3}^{t} \bar{Q}_{2}}}{S_{00} S_{0 \bar{Q}_{2}}} \operatorname{Tr}_{Q_{1}} V_{1} \operatorname{Tr}_{Q^{t}} V_{1}^{-1} \operatorname{Tr}_{Q_{2}} V_{2} \operatorname{Tr}_{Q \otimes Q_{3}} V_{3} .
$$

By large $N$-duality the above amplitude is computed by the topological strings on $X$ with three stacks of D-branes, as in [3], corresponding to the right figure in fig. 7 . In the limit where we send $N$ or equivalently $t=N g_{s}$ to infinity, $Y$ becomes a $\mathbf{C}^{\mathbf{3}}$, and this is the limit we are interested in. All the $N$ dependence in (6.2) is in the $S$-matrices, and in the following we will use $W_{Q_{i} Q_{j}}$ to mean the limit of the matrix $S$-matrix as $N$ goes to infinity, $W_{Q_{i} Q_{j}}=\lim _{t \rightarrow \infty} S_{Q_{j} \bar{Q}_{i}} / S_{00}$ which was introduced in [3]. So, in the limit where $Y$ becomes $\mathbf{C}^{\mathbf{3}}$ the amplitude in (6.2) simply becomes

$$
Z\left(V_{1}, V_{2}, V_{3}\right)=\sum_{Q_{1}, Q_{2}, Q_{3}, Q}(-1)^{\ell\left(Q_{1}\right)} \frac{W_{Q_{2} Q_{1}^{t}} W_{Q_{2} Q_{3}^{t}}}{W_{Q_{2} 0}} \operatorname{Tr}_{Q_{1}} V_{1} \operatorname{Tr}_{Q^{t}} V_{1}^{-1} \operatorname{Tr}_{Q_{2}} V_{2} \operatorname{Tr}_{Q \otimes Q_{3}} V_{3}
$$

Our main interest is in the amplitude where the D-brane on $L_{1}$ is on the first leg of $\mathbf{C}^{3}$. This we will do by a suitable "analytic continuation". The only part of the amplitude in (6.2) that can be affected by moving the D-brane there involves the representation labeled by $Q_{1}$, as this representation corresponds to the world-sheet instanton strings which may become massless in the process. The only other representation that could have been affected is the one labeled by $Q$, however the action of the corresponding strings is growing in the process, and as far as they are concerned, the above expressions are getting more and more reliable. At any rate, we will provide, in the following sections, 

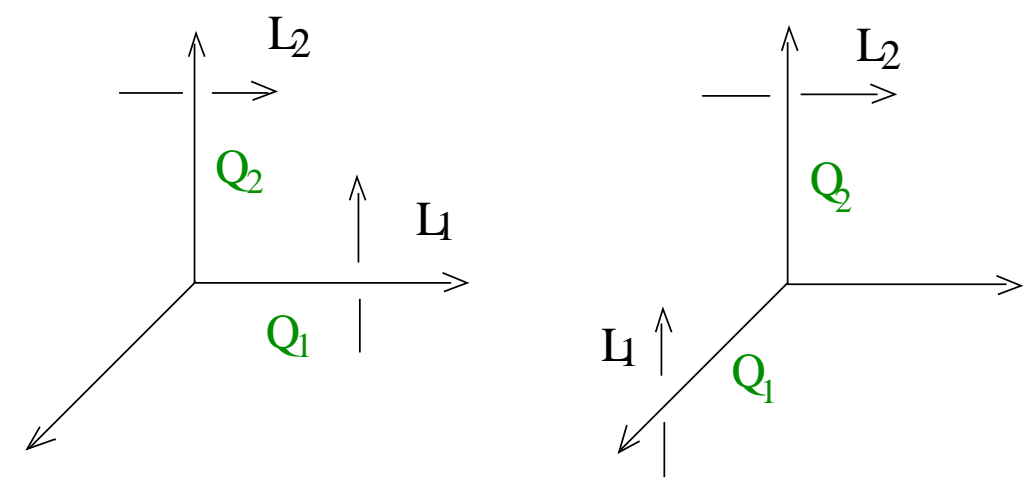

Fig. 11 The left and the right hand side of the figure describe two D-brane configurations in $\mathbf{C}^{3}$, the latter obtained by moving the D-brane $L_{1}$. The amplitude corresponding to configuration on the left is $C_{0, Q_{2}, Q_{1}}^{0,0,-1}$ and on the right it is $C_{Q_{1}, Q_{2}, 0}^{0,0,0}$.

strong evidence that this is correct. With this assumption, we only need to know what the transition means for the part of amplitude corresponding to $W_{Q_{1} Q_{2}}$. To answer this, we may well study a simpler problem where we have only two stacks of D-branes, one wrapping $L_{2}$ and one on $L_{1}$, to start with, as in fig. 11. Now, notice that this amplitude equals precisely

$$
Z\left(V_{1}, V_{2}\right)=\sum_{Q_{1}, Q_{2}} W_{Q_{2} Q_{1}^{t}}(-1)^{\ell\left(Q_{1}\right)} \operatorname{Tr}_{Q_{1}} V_{1} \operatorname{Tr}_{Q_{2}} V_{2}
$$

By using the definition of the three-point vertex, and keeping track of framing, $W_{Q_{2} Q_{1}^{t}}(-1)^{\ell\left(Q_{1}\right)}$ should equal

$$
C_{0, Q_{2}, Q_{1}}^{0,0,-1}=C_{0, Q_{2}, Q_{1}}(-1)^{\ell\left(Q_{2}\right)} q^{-\kappa_{Q_{1} / 2}}
$$

from which we conclude that

$$
C_{0, Q_{2}, Q_{1}}=W_{Q_{2} Q_{1}^{t}} q^{\kappa_{Q_{1}} / 2}
$$

From this we can immediately find that the amplitude corresponding to the second phase in fig. 11. From the definition of the three-point vertex alone, this is $C_{Q_{1}, Q_{2} 0}$, but by cyclic symmetry (3.9) of the vertex and (6.4), $C_{Q_{1}, Q_{2}, 0}$ is the same as $W_{Q_{2}^{t} Q_{1}} q^{\kappa_{Q_{2}}} / 2$ (the reader should recall that $S$, and hence $W$ is symmetric). We conclude that in going from the left to the right hand side of fig. 11 we must replace

$$
W_{Q_{2} Q_{1}^{t}} \operatorname{Tr}_{Q_{1}} V_{1} \operatorname{Tr}_{Q_{2}} V_{2} \rightarrow W_{Q_{2}^{t} Q_{1}} q^{\kappa_{Q_{2}} / 2} \operatorname{Tr}_{Q_{1}} V_{1}^{-1} \operatorname{Tr}_{Q_{2}} V_{2}
$$

in (6.3). The strings ending on the $L_{1}$ D-brane in this new phase, labeled with $Q_{1}$ and will naturally have the same charge on the D-brane as the strings labeled with $Q$ since now 
moving the D-brane affects both of their masses in the same way (recall that $V$ is a phase of a complex field), and this is why we replace $V_{1}$ by $V_{1}^{-1}$ in the above formula. Redefining $V_{1} \rightarrow V_{1}^{-1}$ and collecting the coefficient of $\operatorname{Tr}_{R_{1}} V_{1} \operatorname{Tr}_{R_{2}} V_{2} \operatorname{Tr}_{R_{3}} V_{3}$ in the partition function we compute $C_{R_{1}, R_{2}, R_{3}}^{0,0,-1}$. Correspondingly, we get the following expression for the three-point vertex in the canonical framing $C_{R_{1}, R_{2}, R_{3}}$ :

$$
C_{R_{1}, R_{2}, R_{3}}=\sum_{Q_{1}, Q_{3}} N_{Q_{1} Q_{3}^{t}}^{R_{1} R_{3}^{t}} q^{\kappa_{R_{2}} / 2+\kappa_{R_{3}} / 2} \frac{W_{R_{2}^{t} Q_{1}} W_{R_{2} Q_{3}^{t}}}{W_{R_{2} 0}} .
$$

where $N_{Q_{1} Q_{3}^{t}}^{R_{1} R_{3}^{t}}$ counts the number of ways representations $Q_{1}$ and $Q_{3}^{t}$ go into $R_{1}$ and $R_{3}^{t}$ :

$$
N_{Q_{1} Q_{3}^{t}}^{R_{1}^{t} R_{3}^{t}}=\sum_{Q} N_{Q Q_{1}}^{R_{1}} N_{Q Q_{3}^{t}}^{R_{3}^{t}}
$$

One must be careful to note that $N_{R_{i} R_{j}}^{R_{k}}$ in the formula above are the ordinary tensor product coefficients, and not the Verlinde coefficients

We conjecture that the above expression (6.5) is the exact trivalent vertex amplitude. Here we have motivated this result based on the large $N$ topological string duality, combined with certain plausible assumptions. As we discussed in section 3 on general grounds, the vertex amplitude has a $\mathbf{Z}_{3}$ cyclic symmetry (3.9) and transforms simply under exchanges of pairs of indices (3.12). Moreover, in the previous section, we have computed the leading piece of the vertex amplitude, corresponding to genus zero with up to two holes. The expression (6.5) has none of the symmetries of the vertex amplitude manifest, and checking agreement with (5.9) is highly involved. Nevertheless, the expression (6.5) passes all these checks. We will explicitly demonstrate this in section 8 .

\section{Review of Chern-Simons and topological string amplitudes}

In order to work out some examples of closed and open string amplitudes from the three-point vertex, we need a more precise definition of the quantities appearing in (6.5). In this section we review these ingredients, as well as the integrality properties of open and closed string amplitudes.

5 The careful reader should note that, in writing (6.1) and (6.5), we have used the freedom to scale $V$ to absorb a factor of $(-1)^{\ell(R)}$, into $\operatorname{Tr}_{R} V$. 


\subsection{Review of necessary Chern-Simons theory ingredients}

In the evaluation of the amplitudes we will need the Chern-Simons invariants of the Hopf link in arbitrary representations of $U(N)$. In this section we collect some formulae for these invariants. Recall that in terms of Chern-Simons variables

$$
q=\exp \left(g_{s}\right)=\exp \left(\frac{2 \pi i}{k+N}\right), \quad \lambda=q^{N} .
$$

In the duality with topological string theory [2], we have that $t=N g_{s}$, so $\lambda=\mathrm{e}^{t}$. As a warmup, consider $W_{R} \equiv W_{R 0}$, which is related to the Chern-Simons invariant of the unknot in an arbitrary representation $R$. The invariant of the unknot is given by the quantum dimension of $R$ :

$$
\frac{S_{0 R}}{S_{00}}=\operatorname{dim}_{q} R .
$$

The explicit expression for $\operatorname{dim}_{q} R$ is as follows. Let $R$ be a representation corresponding to a Young tableau with row lengths $\left\{\mu_{i}\right\}_{i=1, \cdots, d(\mu)}$, with $\mu_{1} \geq \mu_{2} \geq \cdots$, and where $d(\mu)$ denotes the number of rows. Define the following $q$-numbers:

$$
\begin{aligned}
{[x] } & =q^{\frac{x}{2}}-q^{-\frac{x}{2}}, \\
{[x]_{\lambda} } & =\lambda^{\frac{1}{2}} q^{\frac{x}{2}}-\lambda^{-\frac{1}{2}} q^{-\frac{x}{2}} .
\end{aligned}
$$

Then, the quantum dimension of $R$ is given by

$$
\operatorname{dim}_{q} R=\prod_{1 \leq i<j \leq d(\mu)} \frac{\left[\mu_{i}-\mu_{j}+j-i\right]}{[j-i]} \prod_{i=1}^{d(\mu)} \frac{\prod_{v=-i+1}^{\mu_{i}-i}[v]_{\lambda}}{\prod_{v=1}^{\mu_{i}}[v-i+d(\mu)]}
$$

The quantum dimension is a Laurent polynomial in $\lambda^{ \pm \frac{1}{2}}$ whose coefficients are rational functions of $q^{ \pm \frac{1}{2}}$.

We are interested in the leading power of $\lambda$ in (7.4). As explained in [3], this power is $\ell / 2$, where $\ell=\sum_{i} \mu_{i}$ is the total number of boxes in the representation $R$, and the coefficient of this power is the rational function of $q^{ \pm \frac{1}{2}}$

$$
W_{R}=q^{\kappa_{R} / 4} \prod_{1 \leq i<j \leq d(\mu)} \frac{\left[\mu_{i}-\mu_{j}+j-i\right]}{[j-i]} \prod_{i=1}^{d(\mu)} \prod_{v=1}^{\mu_{i}} \frac{1}{[v-i+d(\mu)]},
$$

where $\kappa_{R}$ is the framing factor introduced in 
Let us now consider the Hopf link with linking number 1. Its invariant for representations $R_{1}, R_{2}, \mathcal{W}_{R_{1} R_{2}}=S_{R_{1} \bar{R}_{2}} / S_{00}$ is given by

$$
\left(\mathcal{W}_{R_{1} R_{2}}\right)_{U(N)}=q^{\ell_{1} \ell_{2} / N}\left(\mathcal{W}_{R_{1} R_{2}}\right)_{S U(N)}
$$

where $\ell_{i}$ is the total number of boxes in the Young tableau of $R_{i}, i=1,2$. The prefactor $q^{\ell_{1} \ell_{2} / N}$ in (7.6) is a correction which was pointed out in [20], and is due to the fact that the vev $W_{R_{1}, R_{2}}$ has to be computed in the theory with gauge group $U(N)$. The expression we will use for this invariant is the one obtained by Morton and Lukac in [37,38]. Their formula is as follows. Let $\mu$ be a Young tableau, and let $\mu^{\vee}$ denote its transposed tableau (remember that this tableau is obtained from $\mu$ by exchanging rows and columns). The Schur polynomial in the variables $\left(x_{1}, \cdots, x_{N}\right)$ corresponding to $\mu$ (which is the character of the diagonal $S U(N)$ matrix $\left(x_{1}, \cdots, x_{N}\right)$ in the representation corresponding to $\mu$ ), will be denoted by $s_{\mu}$. They can be written in terms of elementary symmetric polynomials $e_{i}\left(x_{1}, \cdots, x_{N}\right), i \geq 1$, as follows [39]:

$$
s_{\mu}=\operatorname{det} M_{\mu}
$$

where

$$
M_{\mu}^{i j}=\left(e_{\mu_{i}^{\vee}+j-i}\right)
$$

$M_{\mu}$ is an $r \times r$ matrix, with $r=d\left(\mu^{\vee}\right)$. To evaluate $s_{\mu}$ we put $e_{0}=1, e_{k}=0$ for $k<0$. The expression (7.7), known sometimes as the Jacobi-Trudy identity, can be formally extended to give the Schur polynomial $s_{\mu}(E(t))$ associated to any formal power series $E(t)=1+\sum_{n=1}^{\infty} a_{i} t^{i}$. To obtain this, we simply use the Jacobi-Trudy formula (7.7), but where $e_{i}$ denote now the coefficients of the series $E(t)$, i.e. $e_{i}=a_{i}$. Morton and Lukac define the series $E_{\emptyset}(t)$ as follows:

$$
E_{\emptyset}(t)=1+\sum_{n=1}^{\infty} c_{n} t^{n},
$$

where the coefficients $c_{n}$ are defined by

$$
c_{n}=\prod_{i=1}^{n} \frac{1-\lambda^{-1} q^{i-1}}{q^{i-1}}
$$

They also define a formal power series associated to a tableau $\mu, E_{\mu}(t)$, as follows:

$$
E_{\mu}(t)=E_{\emptyset}(t) \prod_{j=1}^{d(\mu)} \frac{1+q^{\mu_{j}-j} t}{1+q^{-j} t}
$$


One can then consider the Schur function of the power series $(7.10), s_{\mu}\left(E_{\mu^{\prime}}(t)\right)$, for any pair of tableaux $\mu, \mu^{\prime}$, by expanding $E_{\mu^{\prime}}(t)$ and substituting its coefficients in the JacobiTrudy formula (7.7). It turns out that this Schur function is essentially the invariant we were looking for. More precisely, one has

$$
\mathcal{W}_{R_{1}, R_{2}}(q, \lambda)=\left(\operatorname{dim}_{q} R_{1}\right)(\lambda q)^{\frac{\ell_{2}}{2}} s_{\mu_{2}}\left(E_{\mu_{1}}(t)\right)
$$

where $\mu_{1,2}$ are the tableaux corresponding to $R_{1,2}$, and $\ell_{2}$ is the number of boxes of $\mu_{2}$. More details and examples can be found in [37]. It is easy to see from (7.11) that the leading power in $\lambda$ of $\mathcal{W}_{R_{1}, R_{2}}$ is $\left(\ell_{1}+\ell_{2}\right) / 2$, and its coefficient is given by the leading coefficient of the quantum dimension, (7.5), times a rational function of $q^{ \pm \frac{1}{2}}$ that is given by:

$$
W_{R_{1} R_{2}}(q)=W_{R_{1}} q^{\frac{\ell_{2}}{2}} s_{\mu_{2}}\left(E_{\mu_{1}}^{\text {lead }}(t)\right)
$$

where

$$
E_{\mu}^{\text {lead }}(t)=E_{\emptyset}^{\text {lead }}(t) \prod_{j=1}^{d(\mu)} \frac{1+q^{\mu_{j}-j} t}{1+q^{-j} t}
$$

and

$$
E_{\emptyset}^{\text {lead }}(t)=1+\sum_{n=1}^{\infty} \frac{t^{n}}{\prod_{i=1}^{n}\left(q^{i}-1\right)} .
$$

The above results are for knots and links in the standard framing. The framing can be incorporated as in [20], by simply multiplying the Chern-Simons invariant of a link with components in the representations $R_{1}, \cdots, R_{L}$, by the factor

$$
(-1)^{\sum_{\alpha=1}^{L} p_{\alpha} \ell_{\alpha}} q^{\frac{1}{2} \sum_{\alpha=1}^{L} p_{\alpha} \kappa_{R_{\alpha}}}
$$

where $p_{\alpha}, \alpha=1, \cdots, L$ are integers labeling the choice of framing for each component.

\subsection{Integrality of closed string amplitudes}

In this and the following subsection we recall certain integrality properties that the topological A-model amplitudes posses on general grounds [40]. This allows one to formulate our answers in terms of certain integers which capture BPS degeneracies.

The topological A-model free energy $F(X)$ has the following structure:

$$
F(X)=\sum_{g=0}^{\infty} g_{s}^{2 g-2} F_{g}(t) .
$$


Here, $F_{g}(t)$ is the free energy at genus $g$. It can be computed as a sum over two-homology classes of worldsheet instantons of genus $g$,

$$
F_{g}(t)=\sum_{Q} N_{g, Q} e^{-Q \cdot t},
$$

where the vector $Q \in H_{2}(X, \mathbf{Z})$ labels the homology class, $t$ is a vector of Kähler parameters, and $N_{g, Q}$ are Gromov-Witten invariants. The free energy $F(X)$ can be related to counting of certain BPS states on the Calabi-Yau manifold associated to D2 branes wrapping holomorphic curves in $X$ [40]. The relation follows from the embedding of the topological A-model in type IIA string theory on $X$ and its further embedding in M-theory. Moreover it relies on the target string interpretation of topological string amplitudes [8, 41]. This implies that the free energy has the following form [40]:

$$
F(X)=\sum_{n=1}^{\infty} \sum_{Q \in H_{2}(X)} \sum_{g=0}^{\infty} n_{Q}^{g}\left(2 \sinh \left(n g_{s} / 2\right)\right)^{2 g-2} \frac{e^{-n Q \cdot t}}{n} .
$$

In this formula, $q=e^{g_{s}}, g$ is related to an $S U(2)_{L} \subset S O(4)$ quantum number denoting the spin representation of the particle in $4+1$ dimensions, $Q \cdot t$ is the mass of the BPS state and $n_{Q}^{g}$ is an integer which counts the number of BPS states with quantum numbers $Q$ and $g$.

\subsection{Integrality of open string amplitudes}

The free energy of open strings $F(V)$ is given by the logarithm of a partition function with the structure

$$
Z_{\text {open }}\left(V_{1}, \cdots, V_{L}\right)=\sum_{R_{1}, \cdots, R_{L}} Z_{\left(R_{1}, \cdots, R_{L}\right)} \prod_{\alpha=1}^{L} \operatorname{Tr}_{R_{\alpha}} V_{\alpha} .
$$

We define the generating function $f_{\left(R_{1}, \cdots, R_{L}\right)}(q, \lambda)$ through the following equation:

$$
F(V)=\sum_{n=1}^{\infty} \sum_{R_{1}, \cdots, R_{L}} \frac{1}{n} f_{\left(R_{1}, \cdots, R_{L}\right)}\left(q^{n}, \mathrm{e}^{-n t}\right) \prod_{\alpha=1}^{L} \operatorname{Tr}_{R_{\alpha}} V_{\alpha}^{n}
$$

where $R_{\alpha}$ denote representations of $U(M)$ and we are considering the limit $M \rightarrow \infty$. In this limit we can exchange the basis consisting of product of traces of powers in the fundamental representation, with the trace in arbitrary representations. It was shown in [22], following similar ideas in the closed string case [40], that the open topological strings 
compute the partition function of BPS domain walls in a related superstring theory. This led to the result that $F(V)$ has an integral expansion structure. This result was further refined in [34] where it was shown that the corresponding integral expansion leads to the following formula for $f_{\left(R_{1}, \cdots, R_{L}\right)}(q, \lambda)$ :

$$
\begin{aligned}
& f_{\left(R_{1}, \cdots, R_{L}\right)}(q, \lambda)= \\
& \left(q^{\frac{1}{2}}-q^{-\frac{1}{2}}\right)^{L-2} \sum_{g \geq 0} \sum_{Q} \sum_{R_{1}^{\prime}, R_{1}^{\prime \prime} \cdots, R_{L}^{\prime}, R_{L}^{\prime \prime}} \prod_{\alpha=1}^{L} C_{R_{\alpha} R_{\alpha}^{\prime} R_{\alpha}^{\prime \prime}} S_{R_{\alpha}^{\prime}}(q) N_{\left(R_{1}^{\prime \prime}, \cdots, R_{L}^{\prime \prime}\right), g, Q}\left(q^{\frac{1}{2}}-q^{-\frac{1}{2}}\right)^{2 g} \mathrm{e}^{-Q \cdot t} .
\end{aligned}
$$

In this formula $R_{\alpha}, R_{\alpha}^{\prime}, R_{\alpha}^{\prime \prime}$ label representations of the symmetric group $S_{\ell}$, which can be labeled by a Young tableau with a total of $\ell$ boxes. $C_{R} R^{\prime} R^{\prime \prime}$ are the Clebsch-Gordon coefficients of the symmetric group, and the monomials $S_{R}(q)$ are defined as follows. If $R$ is a hook representation

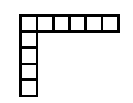

with $\ell$ boxes in total, and with $\ell-d$ boxes in the first row, then

$$
S_{R}(q)=(-1)^{d} q^{-\frac{\ell-1}{2}+d}
$$

and it is zero otherwise. Finally, $N_{\left(R_{1}, \cdots, R_{L}\right), g, Q}$ are integers associated to open string amplitudes. They compute the net number of BPS domain walls of charge $Q$ and spin $g$ transforming in the representations $R_{\alpha}$ of $U(M)$, where we are using the fact that representations of $U(M)$ can also be labeled by Young tableaux. It is also useful to introduce a generating functional for these degeneracies as in [34]:

$$
\widehat{f}_{\left(R_{1}, \cdots, R_{L}\right)}(q, \lambda)=\sum_{g \geq 0} \sum_{Q} N_{\left(R_{1}, \cdots, R_{L}\right), g, Q}\left(q^{\frac{1}{2}}-q^{-\frac{1}{2}}\right)^{2 g} \mathrm{e}^{-Q \cdot t}
$$

We then have the relation:

$$
f_{\left(R_{1}, \cdots, R_{L}\right)}(q, \lambda)=\left(q^{\frac{1}{2}}-q^{-\frac{1}{2}}\right)^{L-2} \sum_{R_{1}^{\prime}, \cdots, R_{L}^{\prime}} M_{R_{1}, \cdots, R_{L} ; R_{1}^{\prime}, \cdots, R_{L}^{\prime}}(q) \widehat{f}_{\left(R_{1}, \cdots, R_{L}\right)}(q, \lambda)
$$

where the matrix $M_{R_{1}, \cdots, R_{L} ; R_{1}^{\prime}, \cdots, R_{L}^{\prime}}(q)$ is given by

$$
M_{R_{1}, \cdots, R_{L} ; R_{1}^{\prime}, \cdots, R_{L}^{\prime}}(q)=\prod_{\alpha=1}^{L} \sum_{R_{\alpha}^{\prime \prime}} C_{R_{\alpha} R_{\alpha}^{\prime} R_{\alpha}^{\prime \prime}} S_{R_{\alpha}^{\prime \prime}}(q)
$$


and it is invertible [34]. Finally, it is also useful sometimes to write BPS degeneracies in the winding number basis:

$$
n_{\left(\vec{k}^{(1)}, \cdots, \vec{k}^{(L)}\right), g, Q}=\sum_{R_{1}, \cdots, R_{L}} \prod_{\alpha=1}^{L} \chi_{R_{\alpha}}\left(C\left(\vec{k}^{(\alpha)}\right)\right) N_{\left(R_{1}, \cdots, R_{L}\right), g, Q} .
$$

Notice that the BPS degeneracies $N_{\left(R_{1}, \cdots, R_{L}\right), g, Q}$ in the representation basis are more fundamental than the degeneracies in the winding number basis, as emphasized in [34].

The $f_{\left(R_{1}, \cdots, R_{L}\right)}$ introduced in $(7.18)$ can be extracted from $Z_{\left(R_{1}, \cdots, R_{L}\right)}$ through a procedure spelled out in detail in [33,34,42]. One has, for example,

$$
f_{\square, \square}=Z_{\square, \square}-Z_{\square, \cdot} \cdot Z_{, \square} .
$$

It is also convenient to introduce the quantities

$$
Z_{\left(\vec{k}^{(1)}, \cdots, \vec{k}^{(L)}\right)}=\sum_{R_{\alpha}} \prod_{\alpha=1}^{L} \chi_{R_{\alpha}}\left(\vec{k}^{(\alpha)}\right) Z_{\left(R_{1}, \cdots, R_{L}\right)}
$$

in such a way that

$$
Z_{\text {open }}\left(V_{1}, \cdots, V_{L}\right)=\sum_{\vec{k}^{(1)}, \cdots, \vec{k}^{(L)}} Z_{\left.\vec{k}^{(1)}, \cdots, \vec{k}^{(L)}\right)} \prod_{\alpha=1}^{L} \frac{1}{z_{\vec{k}^{(\alpha)}}} \Upsilon_{\vec{k}^{(\alpha)}}\left(V_{\alpha}\right) .
$$

We can now write the total free energy as:

$$
F(V)=\sum_{g=0}^{\infty} \sum_{\vec{k}^{(\alpha)}} g_{s}^{2 g-2+h} F_{g, \vec{k}^{(\alpha)}}(t) \prod_{\alpha=1}^{L} \Upsilon_{\vec{k}^{(\alpha)}}\left(V_{\alpha}\right),
$$

where $h=\sum_{\alpha} h_{\alpha}$ is the total number of holes. We have then that,

$$
\sum_{g=0}^{\infty} F_{g, \vec{k}^{(\alpha)}}(t) g_{s}^{2 g-2+h}=\frac{1}{\prod_{\alpha=1}^{L} z_{\vec{k}^{(\alpha)}}} Z_{\vec{k}^{(\alpha)}}^{(c)}
$$

where $(c)$ denotes the connected piece.

\section{The vertex amplitude}

In this section we use the apparatus developed above to calculate some values of the vertex amplitude. This will provide highly non-trivial checks that the vertex amplitude derived in section 6 using large $\mathrm{N}$-dualities is in fact the correct expression. 
As discussed in the previous section, we can extract the free energy of the vertex amplitude with fixed winding numbers as the connected part of $C_{\vec{k}_{1}} \vec{k}_{2} \vec{k}_{3}$ :

$$
F_{\vec{k}^{(1)}, \vec{k}^{(2)}, \vec{k}^{(3)}}^{\left(n_{1}, n_{2}, n_{3}\right)}\left(g_{s}\right)=\frac{1}{\prod_{\alpha=1}^{3} z_{\vec{k}^{(\alpha)}}}\left(C^{(c)}\right)_{\vec{k}^{(1)}, \vec{k}^{(2)}, \vec{k}^{(3)}}^{\left(n_{1}, n_{2}, n_{3}\right)} .
$$

Consider the part of this amplitude corresponding to a single hole on each of the three stacks of D-branes. Since only the winding numbers remain to be specified, we can simply denote this by $F_{k, l, m}$, corresponding to $\vec{k}^{(i)}, i=1,2,3$ with a single nonzero entry in positions $k, l, m$, respectively. Then, one has the following formulae:

$$
\begin{aligned}
& F_{k, 0,0}^{(n, 0,0)}=\frac{1}{k} \frac{[k+n k-1] !}{[k] ![n k] !} \\
& F_{k, l, 0}^{(0,0,0)}=\frac{(-1)^{l+1}}{k l} \frac{[k l]}{[k]}\left[\begin{array}{l}
k \\
l
\end{array}\right],
\end{aligned}
$$

where the $q$-number is $[x]=q^{\frac{x}{2}}-q^{-\frac{x}{2}}$, the $q$-factorial is given by

$$
[x] !=[x][x-1] \cdots[1]
$$

and finally the $q$-combinatorial number is defined as

$$
\left[\begin{array}{l}
x \\
y
\end{array}\right]=\frac{[x] !}{[x-y] ![y] !}
$$

Note that the leading $g_{s}$ terms $F_{k, 0,0}$ and $F_{k, m, 0}$ are

$$
\begin{aligned}
& F_{g=0 ; k, 0,0}^{(n, 0,0)}=\frac{1}{g_{s} k} \frac{(k+n k-1) !}{(k) !(n k) !} \\
& F_{g=0 ; k, l, 0}^{(0,0,0)}=\frac{(-1)^{l+1}}{k}\left(\begin{array}{c}
k \\
l
\end{array}\right),
\end{aligned}
$$

and these agree with (5.10) and (5.9) respectively up to a choice of coordinate, $\operatorname{Tr} V_{i}^{m} \rightarrow$ $(-1)^{m} \operatorname{Tr} V_{i}^{m}$ and the over-all sign of the free energy!

Let us now look at some explicit values of the vertex (6.5) which we can easily compute using the explicit expressions for $W_{R_{1} R_{2}}$, that we gave in the previous section. Using explicit evaluation of $C$ one can verify that at least for small number of boxes the highly non-trivial symmetry prediction (3.9) and (3.12) are indeed satisfied.

We give here a list of values for the trivalent vertex up to five boxes in total. For the sake of space, we mostly list values which are not related by symmetries, although we 
have included some to make manifest the properties that we derived in section 3 . The dot - stands for the trivial representation.

$$
\begin{aligned}
& C_{\text {口.. }}=\frac{1}{q^{\frac{1}{2}}-q^{-\frac{1}{2}}}, \\
& C_{\text {口ロ }}=\frac{q^{2}-q+1}{(q-1)^{2}}, \quad C_{\text {口.. }}=\frac{q^{2}}{(q-1)\left(q^{2}-1\right)}, \\
& C_{\text {日.. }}=\frac{q}{(q-1)\left(q^{2}-1\right)}, \\
& C_{\text {口ロ }}=\frac{q^{4}-q^{3}+q^{2}-q+1}{q^{\frac{1}{2}}(q-1)^{3}}, \quad C_{\text {口ロ. }}=\frac{q^{\frac{3}{2}}\left(q^{3}-q^{2}+1\right)}{(q-1)^{2}\left(q^{2}-1\right)}, \\
& C_{\text {日丁. }}=\frac{q^{3}-q^{2}+1}{q^{\frac{1}{2}}(q-1)^{2}\left(q^{2}-1\right)}, \quad C_{\text {口س.. }}=\frac{q^{\frac{9}{2}}}{(q-1)\left(q^{2}-1\right)\left(q^{3}-1\right)} ， \\
& C_{\boxminus . .}=\frac{q^{\frac{5}{2}}}{(q-1)^{2}\left(q^{3}-1\right)}, \quad C_{\text {日.. }}=\frac{q^{\frac{3}{2}}}{(q-1)\left(q^{2}-1\right)\left(q^{3}-1\right)} ，
\end{aligned}
$$

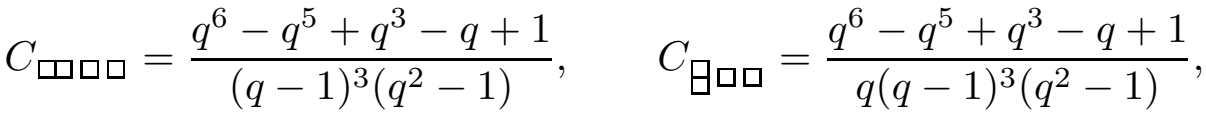

$$
\begin{aligned}
& C_{\text {口ロ }}=\frac{q^{2}\left(q^{4}-q^{2}+1\right)}{(q-1)^{2}\left(q^{2}-1\right)^{2}}, \quad C_{\text {口曰. }}=\frac{q\left(q^{6}-q^{5}-q^{4}+2 q^{3}-q+1\right)}{(q-1)^{2}\left(q^{2}-1\right)^{2}},
\end{aligned}
$$

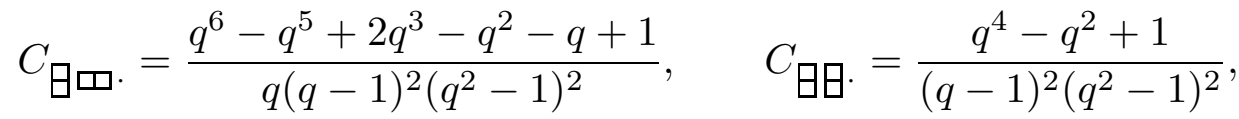

$$
\begin{aligned}
& C_{\text {Шロ }}=\frac{q^{4}\left(q^{4}-q^{3}+1\right)}{(q-1)^{2}\left(q^{2}-1\right)\left(q^{3}-1\right)}, \quad C_{\text {Ð口. }}=\frac{q\left(q^{4}-q^{3}+q^{2}-q+1\right)}{(q-1)^{3}\left(q^{3}-1\right)} ， \\
& C_{\text {目口 }}=\frac{q^{4}-q+1}{q(q-1)^{2}\left(q^{2}-1\right)\left(q^{3}-1\right)}, \\
& C_{\text {口四. }}=\frac{q^{8}}{(q-1)\left(q^{2}-1\right)\left(q^{3}-1\right)\left(q^{4}-1\right)}, \quad C_{\text {甲... }}=\frac{q^{5}}{(q-1)^{2}\left(q^{2}-1\right)\left(q^{4}-1\right)}, \\
& C_{\text {田. }}=\frac{q^{4}}{(q-1)^{2}\left(q^{2}-1\right)\left(q^{4}-1\right)}, \quad C_{\text {曰.. }}=\frac{q^{3}}{(q-1)^{2}\left(q^{2}-1\right)\left(q^{4}-1\right)}, \\
& C_{\text {目. }}=\frac{q^{2}}{(q-1)\left(q^{2}-1\right)\left(q^{3}-1\right)\left(q^{4}-1\right)}, \\
& C_{\text {ロロ }}=\frac{q^{\frac{1}{2}}\left(q^{8}-q^{7}+q^{5}-q^{4}+q^{3}-q+1\right)}{(q-1)^{3}\left(q^{2}-1\right)^{2}},
\end{aligned}
$$




$$
\begin{aligned}
& C_{\text {Ш日口 }}=\frac{q^{9}-q^{8}-q^{7}+2 q^{6}-q^{4}+q^{3}-q+1}{q^{\frac{1}{2}}(q-1)^{3}\left(q^{2}-1\right)^{2}}, \\
& C_{\text {日田 }}=\frac{q^{9}-q^{8}+q^{6}-q^{5}+2 q^{3}-q^{2}-q+1}{q^{\frac{3}{2}}(q-1)^{3}\left(q^{2}-1\right)^{2}}, \\
& C_{\text {日日 }}=\frac{q^{8}-q^{7}+q^{5}-q^{4}+q^{3}-q+1}{q^{\frac{3}{2}}(q-1)^{3}\left(q^{2}-1\right)^{2}}, \\
& C_{\text {四口吅 }}=\frac{q^{\frac{3}{2}}\left(q^{8}-q^{7}+q^{4}-q+1\right)}{(q-1)^{3}\left(q^{2}-1\right)\left(q^{3}-1\right)}, \\
& C_{\text {Ð }}=\frac{q^{8}-2 q^{7}+3 q^{6}-3 q^{5}+3 q^{4}-3 q^{3}+3 q^{2}-2 q+1}{q^{\frac{1}{2}}(q-1)^{4}\left(q^{3}-1\right)}, \\
& C_{\text {目口吅 }}=\frac{q^{8}-q^{7}+q^{4}-q+1}{q^{\frac{3}{2}}(q-1)^{3}\left(q^{2}-1\right)\left(q^{3}-1\right)}, \\
& C_{\text {Шس丨 }}=\frac{q^{\frac{9}{2}}\left(q^{5}-q^{3}+1\right)}{(q-1)^{2}\left(q^{2}-1\right)^{2}\left(q^{3}-1\right)}, \\
& C_{\text {四 }}=\frac{q^{\frac{7}{2}}\left(q^{8}-q^{7}-q^{6}+q^{5}+q^{4}-q^{2}+1\right)}{(q-1)^{2}\left(q^{2}-1\right)^{2}\left(q^{3}-1\right)}, \\
& C_{\text {甲コ. }}=\frac{q^{\frac{1}{2}}\left(q^{7}-q^{6}+q^{4}-q+1\right)}{(q-1)^{3}\left(q^{2}-1\right)\left(q^{3}-1\right)}, \\
& C_{\text {曰日. }}=\frac{q^{\frac{1}{2}}\left(q^{7}-q^{6}+q^{3}-q+1\right)}{(q-1)^{3}\left(q^{2}-1\right)\left(q^{3}-1\right)}, \\
& C_{\text {日四. }}=\frac{q^{8}-q^{6}+q^{4}+q^{3}-q^{2}-q+1}{q^{\frac{5}{2}}(q-1)^{2}\left(q^{2}-1\right)^{2}\left(q^{3}-1\right)}, \\
& C_{\text {日旺. }}=\frac{q^{5}-q^{2}+1}{q^{\frac{1}{2}}(q-1)^{2}\left(q^{2}-1\right)^{2}\left(q^{3}-1\right)}, \\
& C_{\text {سم口 }}=\frac{q^{\frac{15}{2}}\left(q^{5}-q^{4}+1\right)}{(q-1)^{2}\left(q^{2}-1\right)\left(q^{3}-1\right)\left(q^{4}-1\right)}, \\
& C_{\text {由吕 }}=\frac{q^{\frac{7}{2}}\left(q^{5}-q^{4}+q^{2}-q+1\right)}{(q-1)^{3}\left(q^{2}-1\right)\left(q^{4}-1\right)}, \\
& C_{\text {田 }}=\frac{q^{\frac{5}{2}}\left(q^{4}-q^{2}+1\right)}{(q-1)^{2}\left(q^{2}-1\right)^{2}\left(q^{3}-1\right)}, \\
& C_{\text {曰品 }}=\frac{q^{\frac{1}{2}}\left(q^{5}-q^{4}+q^{3}-q+1\right)}{(q-1)^{3}\left(q^{2}-1\right)\left(q^{4}-1\right)} ， \\
& C_{\text {目口. }}=\frac{q^{5}-q+1}{q^{\frac{3}{2}}(q-1)^{2}\left(q^{2}-1\right)\left(q^{3}-1\right)\left(q^{4}-1\right)}, \\
& C_{\text {酉. }}=\frac{q^{\frac{25}{2}}}{(q-1)\left(q^{2}-1\right)\left(q^{3}-1\right)\left(q^{4}-1\right)\left(q^{5}-1\right)},
\end{aligned}
$$




$$
\begin{aligned}
& C_{\text {巴⿴囗. }}=\frac{q^{\frac{17}{2}}}{(q-1)^{2}\left(q^{2}-1\right)\left(q^{3}-1\right)\left(q^{5}-1\right)}, \\
& C_{\text {巴.. }}=\frac{q^{\frac{13}{2}}}{(q-1)^{2}\left(q^{2}-1\right)\left(q^{3}-1\right)\left(q^{4}-1\right)}, \\
& C_{\text {目.. }}=\frac{q^{\frac{11}{2}}}{(q-1)^{2}\left(q^{2}-1\right)^{2}\left(q^{5}-1\right)} ， \\
& C_{\text {Ð.. }}=\frac{q^{\frac{9}{2}}}{(q-1)^{2}\left(q^{2}-1\right)\left(q^{3}-1\right)\left(q^{4}-1\right)} ， \\
& C_{\text {目.. }}=\frac{q^{\frac{7}{2}}}{(q-1)^{2}\left(q^{2}-1\right)\left(q^{3}-1\right)\left(q^{5}-1\right)} ， \\
& C_{\text {目. }}=\frac{q^{\frac{5}{2}}}{(q-1)\left(q^{2}-1\right)\left(q^{3}-1\right)\left(q^{4}-1\right)\left(q^{5}-1\right)} .
\end{aligned}
$$

Note for example that:

$$
C_{\text {口田 }}=q^{3} C_{\text {目口口 }},
$$

while on the other hand $k_{\text {س }}=6$, so that this precisely agrees with (3.12).

The vertex amplitude, being an open string amplitude, has to satisfy strong integrality requirements that we have reviewed in section 6 . In order to check this, we can compute the generating functionals for BPS states $\widehat{f}_{R_{1} R_{2} R_{3}}$ for arbitrary framings in the legs. If we denote $z=\left(q^{\frac{1}{2}}-q^{-\frac{1}{2}}\right)^{2}$, one finds, for example:

$$
\begin{aligned}
& \widehat{f}_{\square, \square, \square}=(-1)^{n_{1}+n_{2}+n_{3}} \text {, } \\
& \widehat{f}_{\square, \square, \square}=-(-1)^{n_{2}+n_{3}}\left(\frac{q^{\frac{n_{1}}{2}}-q^{-\frac{n_{1}}{2}}}{q^{\frac{1}{2}}-q^{-\frac{1}{2}}}\right)^{2} \\
& =-(-1)^{n_{2}+n_{3}} n_{1}^{2}-\frac{1}{12}(-1)^{n_{2}+n_{3}} n_{1}^{2}\left(n_{1}^{2}-1\right) z+\cdots,
\end{aligned}
$$

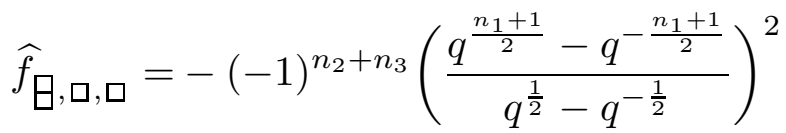

$$
\begin{aligned}
& =-(-1)^{n_{2}+n_{3}}\left(1+n_{1}\right)^{2}-\frac{1}{12}(-1)^{n_{2}+n_{3}} n_{1}\left(n_{1}+1\right)^{2}\left(n_{1}+2\right) z+\cdots,
\end{aligned}
$$

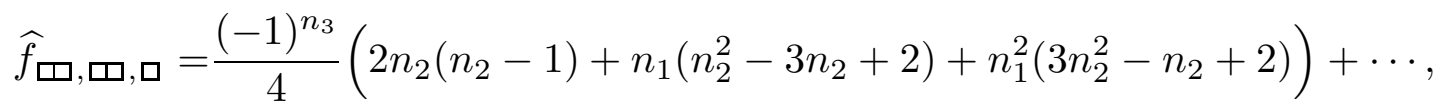

$$
\begin{aligned}
& \widehat{f}_{\text {口,日, 口 }}=\frac{(-1)^{n_{3}}}{4}\left(n_{1} n_{2}\left(n_{2}-1\right)+2 n_{2}\left(1+n_{2}\right)+n_{1}^{2}\left(3 n_{2}^{2}+5 n_{2}+4\right)\right)+\cdots, \\
& \widehat{f}_{\text {日, Ш, 口 }}=\frac{(-1)^{n_{3}}}{4}\left(4-6 n_{2}+6 n_{2}^{2}+n_{1}\left(7 n_{2}^{2}-5 n_{2}+6\right)+n_{1}^{2}\left(3 n_{2}^{2}-n_{2}+2\right)\right)+\cdots, \\
& \widehat{f}_{\text {日,日, }}=\frac{(-1)^{n_{3}}}{4}\left(4+6 n_{2}+6 n_{2}^{2}+n_{1}\left(7 n_{2}^{2}+9 n_{2}+8\right)+n_{1}^{2}\left(3 n_{2}^{2}+5 n_{2}+4\right)\right)+\cdots,
\end{aligned}
$$


This passes the integrality check.

\section{Examples of open and closed string amplitudes from the vertex.}

In this section we compute various closed and open string amplitudes using the trivalent vertex. In the examples below we have made many checks of the vertex against amplitudes of closed and open string calculations using other means.

In the closed string case the vertex can be checked, in principle to all genera, by comparison with mirror B-model calculations using holomorphic anomaly [8], see [43, 44, 29], as well as against A-model localisation calculations using the techniques of [16,45,46]. More directly one can compare the vertex with open string amplitudes. B-model calculations for the open string were so far only available for the disk following [19,11]. A-model localization calculations for all genus open string amplitudes with a single stack of D-branes have been introduced in [47,45]. At the operational level this is a minor modification of the localization procedure of the closed string case, and we provide a computer program which computes this for general toric configurations 6 . Due to the extended combinatorics of the graphs indexing the fixed points of the torus action on the moduli space of stable maps [16,45, 46, the computer calculation is very slow compared with the techniques developed in the present paper, in particular for amplitudes with higher genus and larger degree (w.r.t the compact Kähler classes). A-model calculations provide, on the other hand, expressions which describe all windings. While the results of the calculations have been checked for many cases, see in particular [45], the procedure has not been established rigorously and leaves interesting conceptual issues to be developed, in particular in regards to multiple stacks of branes. It should be possible to derive within the localization approach general expressions for the vertex for general windings and framings. Some attmepts in this directions have already been made [7].

To begin with, it is useful to see how the vertex works in a few simple examples where the complete amplitudes are known.

\subsection{Example I}

6 This program can be distributed on request. It requires the evaluation of 2 d gravity correlations functions, which where implemented in Maple by Carel Faber, see also [48. 

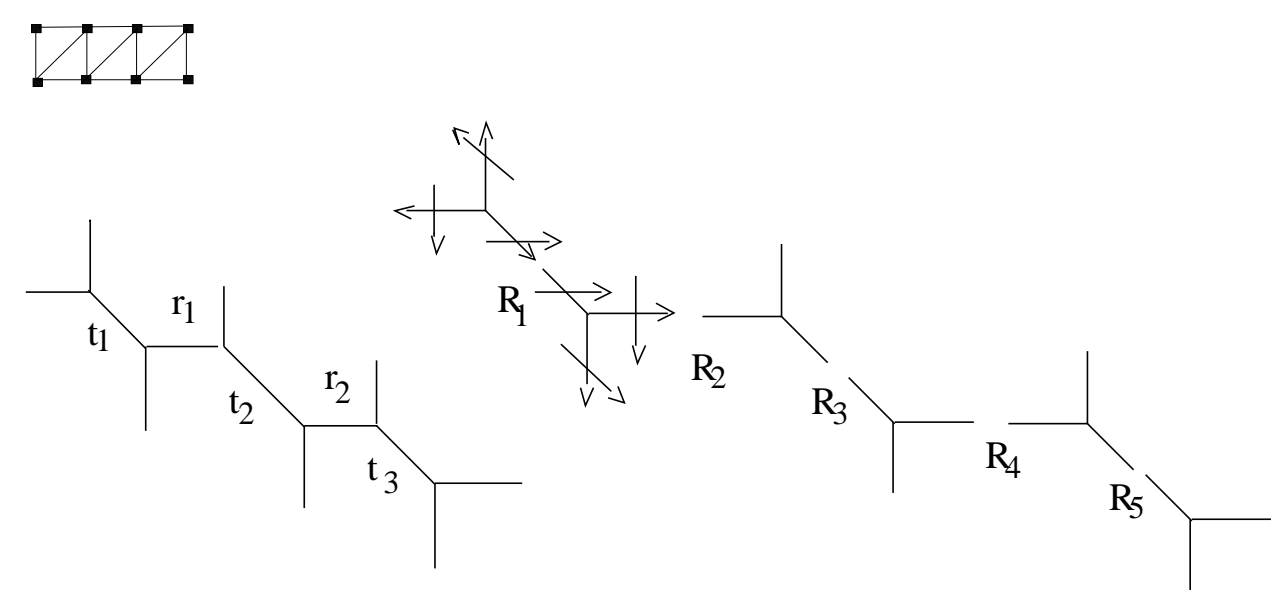

Fig. 12 The left hand side is the graph $\Gamma$ of a geometry containing a chain of $\mathbf{P}^{\mathbf{1}}$ 's with five independent classes in $\mathrm{H}_{2}$. The right hand side depicts a decomposition of this graph in terms of three-point vertices. All the vertices are obvious repetitions of the first two, moreover, all the vertex amplitudes equal $C_{R_{1} R_{2} R_{3}}$ with representation ordered cyclically, counter-clockwise. Some of the representations are set to be trivial, as corresponding legs are non-compact. The small figure in the upper left corner is the corresponding graph $\hat{\Gamma}$.

The closed string amplitude in fig. 12 can be written in terms of six trivalent vertices glued together. Two of them are of the kind we have already discussed. Using an $S L(2, \mathbf{Z})$ transformation, we find the differently oriented trivalent vertex corresponding to fig. 12 .

From this, and using the gluing rules above, we find that the closed string amplitude can be written as

$$
\begin{array}{r}
Z=\sum_{R_{1}, \cdots, R_{5}}(-1)^{\sum_{i} \ell\left(R_{i}\right)} C_{\cdot \cdot R_{1}} e^{-\ell\left(R_{1}\right) t_{1}} C_{\cdot R_{1}^{t} R_{2}} e^{-\ell\left(R_{2}\right) r_{1}} C_{R_{2}^{t} \cdot R_{3}} \\
\times e^{-\ell\left(R_{3}\right) t_{2}} C_{\cdot R_{3}^{t} R_{4}} e^{-\ell\left(R_{4}\right) r_{2}} C_{R_{4}^{t} \cdot R_{5}} e^{-\ell\left(R_{5}\right) t_{3}} C \cdot R_{5}^{t},
\end{array}
$$

Note that to get the all genus answer up to degree $n$ in any one of the five classes, we only need to perform the sum over the corresponding representation with up to $n$ boxes. It is not difficult to check that this is the correct A-model amplitude on $X$, which is known to all genera. For example, one way to calculate the amplitude is to use mirror symmetry to calculate the genus zero amplitude, and integrality to fix the full free energy. This is possible as there are no curves in $X$ with genus higher than zero, so we find

$$
Z=\exp \left[\sum_{n} \frac{N_{\vec{Q}} e^{-n \vec{Q} \cdot \vec{t}}}{n\left(2 \sinh \left(n g_{s} / 2\right)\right)^{2}}\right]
$$


where $N_{\vec{Q}}$ are the degeneracies of BPS states corresponding to a $\mathbf{P}^{\mathbf{1}}$ in class $\vec{Q}$ in $X$ whose values are as follows. First, there can be no BPS states corresponding to $\mathbf{P}^{\mathbf{1}}$ 's which are disconnected. For the chains of connected $\mathbf{P}^{\mathbf{1}}$ 's we have that: $N_{\vec{Q}}=-1$ if $\vec{Q}$ corresponds to the class of an odd number of connected $\mathbf{P}^{\mathbf{1}}$ 's in $X$ (e.g. BPS states with masses $r_{1}$, $r_{1}+t_{2}+r_{2}$ and $t_{1}+r_{1}+t_{2}+r_{2}+t_{3}$ all have $\left.N_{\vec{Q}}=-1\right), N_{\vec{Q}}=1$ if $\vec{Q}$ corresponds to a class of an even number of connected $\mathbf{P}^{1}$ 's (e.g. $r_{2}+t_{2}$ and $\left.r_{1}+t_{2}+{ }_{2}+t_{3}\right)$.

\subsection{Example II}

It is also easy to see that our rules reproduce the $\mathcal{O}(-3) \rightarrow \mathbf{P}^{\mathbf{2}}$ amplitudes computed in [3]. In [3], the all genus amplitude was computed using a quiver-type Chern-Simons theory with three nodes $G=U\left(N_{1}\right) \times U\left(N_{2}\right) \times U\left(N_{3}\right)$ and bifundamental matter-fields, in the $N_{i} \rightarrow \infty$ limit.

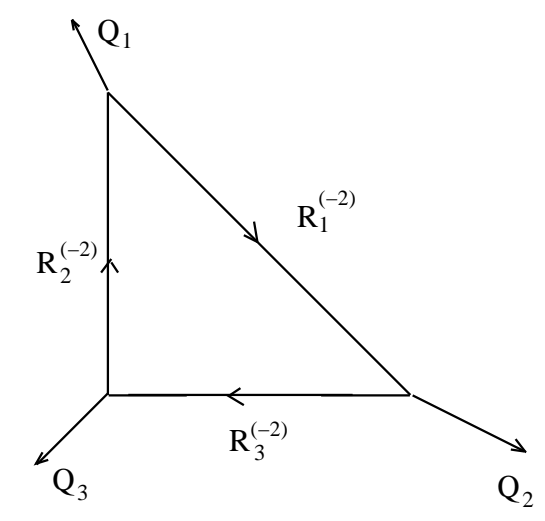

Fig. 13 The trivalent vertices for gluing to $\mathcal{O}(-3) \rightarrow \mathbf{P}^{\mathbf{2}}$ amplitude (with D-branes on external legs). The superscript on the representations give the framing $n$ in the corresponding propagator.

Using $S L(2, \mathbf{Z})$ transformations and adjusting framings appropriately, it is easy to see that the amplitude corresponding to the graph in fig. 13, when there are no branes in the outer legs, can be written as

$$
Z_{\mathbf{P}^{\mathbf{2}}}=\sum_{R_{1}, R_{2}, R_{3}}(-1)^{\sum_{i} \ell\left(R_{i}\right)} e^{-\sum_{i} \ell\left(R_{i}\right) t} q^{\sum_{i} \kappa_{R_{i}}} C_{R_{2} R_{3}^{t}} C \cdot R_{1} R_{2}^{t} C \cdot R_{3} R_{1}^{t},
$$

where $t$ is the Kähler parameter of $\mathcal{O}(-3) \rightarrow \mathbf{P}^{\mathbf{2}}$. Using the fact that, e.g.

$$
C \cdot R_{2} R_{3}^{t}=W_{R_{2} R_{3}} q^{-\kappa_{R_{3}} / 2}
$$

the amplitude (9.2) precisely equals the amplitude obtained in [3] by related, but different methods.

\subsection{Example III}




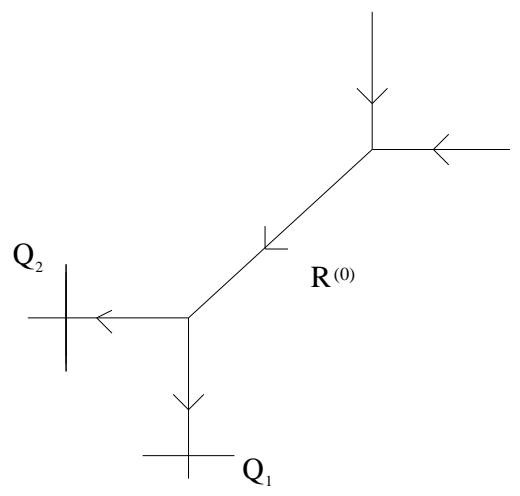

Fig. 14 This shows $\mathcal{O}(-1)+\mathcal{O}(-1) \rightarrow \mathbf{P}^{\mathbf{1}}$ with D-branes on external legs

Another example where we can use the full vertex amplitude is shown in fig. 14: two D-branes on the outer legs of $\mathcal{O}(-1)+\mathcal{O}(-1) \rightarrow \mathbf{P}^{\mathbf{1}}$. According to the gluing rules we have

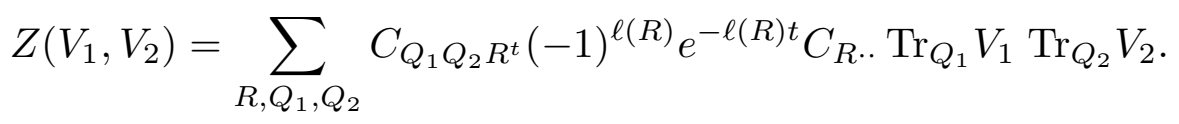

On the other hand, this amplitude corresponds to a Hopf link inside $\mathbf{S}^{3}$ with linking number -1 , therefore we should have

$$
Z\left(V_{1}, V_{2}\right)=\sum_{Q_{1}, Q_{2}} \lambda^{-\frac{\ell_{Q_{1}}+\ell_{Q_{2}}}{2}} S_{Q_{1} \bar{Q}_{2}} \operatorname{Tr}_{Q_{1}} V_{1} \operatorname{Tr}_{Q_{2}} V_{2}
$$

where $\lambda=e^{-t}$. One can check that indeed (9.3) and (9.4) agree. Namely, we have that

$$
S_{Q_{1} \bar{Q}_{2}}=\mathcal{W}_{Q_{1} Q_{2}}(q, \lambda) S_{00}(\lambda)
$$

where $\mathcal{W}_{Q_{1} Q_{2}}$ is the invariant calculated in (7.11), and $S_{00}$ is the partition function of CS on $\mathbf{S}^{3}$, which can be written as] [2]

$$
S_{00}\left(\mathrm{e}^{-t}\right)=\exp \left(-\sum_{k=1}^{\infty} \frac{\mathrm{e}^{-k t}}{k\left(q^{\frac{k}{2}}-q^{-\frac{k}{2}}\right)^{2}}\right) .
$$

For example, for $Q_{1}=R=\square$, agreement requires that

$$
C_{\square Q \square}=W_{Q \square} W_{\square}-\frac{W_{Q \square}^{(1)}}{W_{\square}},
$$

7 There are pieces in the free energy which are finite polynomials in $t$ which encode certain topological data in the compact case. In the non-compact case at hand they are ambiguous and in our vertex amplitudes we have naturally set them to zero. 
where $W_{Q_{1} Q_{2}}^{(1)}$ is defined by the expansion

$$
\mathcal{W}_{Q_{1} Q_{2}}(q, \lambda)=\lambda^{\frac{\ell_{Q_{1}}+\ell_{Q_{2}}}{2}} W_{Q_{1} Q_{2}}(q)+\lambda^{\frac{\ell_{Q_{1}}+\ell_{Q_{2}}}{2}-1} W_{Q_{1} Q_{2}}^{(1)}(q)+\cdots
$$

Using the explicit formula (7.11), we find that

$$
C_{\square Q \square}=q^{\kappa_{Q} / 2} \frac{W_{\square Q^{t}} W_{\square Q}}{W_{Q}}+W_{Q},
$$

in agreement with (6.5).

\subsection{Example $I V$}

Another non-trivial configuration involving the full trivalent vertex for which we have an immediate prediction is $\mathcal{O}(-3) \rightarrow \mathbf{P}^{\mathbf{2}}$ with "outer" D-branes on the external legs. This corresponds to the amplitude (9.2) but where we allow non-trivial external representations on the trivalent vertices:

$$
\begin{aligned}
& Z_{\mathbf{P}^{2}}\left(V_{1}, V_{2}, V_{3}\right)= \\
& \sum_{R_{i}, Q_{i}} C_{Q_{3} R_{2} R_{3}^{t}} C_{Q_{1} R_{1} R_{2}^{t}} C_{Q_{2} R_{3} R_{1}^{t}}(-1)^{\sum_{i} \ell\left(R_{i}\right)} e^{-\sum_{i} \ell\left(R_{i}\right) t} q^{\sum_{i} \kappa_{R_{i}} \operatorname{Tr}_{Q_{1}} V_{1} \operatorname{Tr}_{Q_{2}} V_{2} \operatorname{Tr}_{Q_{3}} V_{3} .}
\end{aligned}
$$

This amplitude is the product of the closed string amplitude $Z_{\mathbf{P}^{\mathbf{2}}}$ given in (9.2), and the open string amplitude properly speaking, so we will write

$$
Z_{\text {open }}\left(V_{1}, V_{2}, V_{3}\right)=\frac{Z_{\mathbf{2}}\left(V_{1}, V_{2}, V_{3}\right)}{Z_{\mathbf{P}^{2}}}=\sum_{Q_{i}} Z_{\left(Q_{1}, Q_{2}, Q_{3}\right)}\left(q, e^{-t}\right) \operatorname{Tr}_{Q_{1}} V_{1} \operatorname{Tr}_{Q_{2}} V_{2} \operatorname{Tr}_{Q_{3}} V_{3}
$$

Notice that the amplitudes are completely symmetric in $Q_{1}, Q_{2}, Q_{3}$, as they should be by the symmetry of the geometry.

The generating functions $\widehat{f}_{\left(Q_{1}, Q_{2}, Q_{3}\right)}$ are computed from $Z_{\left(Q_{1}, Q_{2}, Q_{3}\right)}$. Let us denote $z=\left(q^{\frac{1}{2}}-q^{-\frac{1}{2}}\right)^{2}, y=e^{-t}$, so that

$$
\widehat{f}_{\left(Q_{1}, Q_{2}, Q_{3}\right)}=\sum_{g, Q} N_{\left(R_{1}, R_{2}, R_{3}\right), g, Q} z^{g} y^{Q}
$$

where $N_{\left(R_{1}, R_{2}, R_{3}\right), g, Q}$ are the degeneracies of BPS states with the corresponding charges. We find, for the first few representations and up to degree five in the Kähler parameter, 
the following results:

$$
\begin{aligned}
& \widehat{f}_{\square, \cdot, \cdot}=1-2 y+5 y^{2}-(32+9 z) y^{3}+\left(286+288 z+108 z^{2}+14 z^{3}\right) y^{4} \\
& -\left(3038+6984 z+7506 z^{2}+4519 z^{3}+1542 z^{4}+276 z^{5}+20 z^{6}\right) y^{5}+\cdots, \\
& \widehat{f}_{\square, \square, \cdot}=-y+4 y^{2}-(35+8 z) y^{3}+\left(400+344 z+112 z^{2}+13 z^{3}\right) y^{4} \\
& -\left(5187+10504 z+10036 z^{2}+5434 z^{3}+1691 z^{4}+280 z^{5}+19 z^{6}\right) y^{5}+\cdots, \\
& \widehat{f}_{\text {Ш }, \cdot, \cdot}=7 y^{3}-\left(110+68 z+12 z^{2}\right) y^{4} \\
& +\left(1651+2938 z+2353 z^{2}+992 z^{3}+212 z^{4}+18 z^{5}\right) y^{5}+\cdots \\
& \widehat{f}_{\text {日, }, \cdot}=y-4 y^{2}+\left(28+8 z^{2}\right) y^{3}-\left(290+276 z+100 z^{2}+13 z^{3}\right) y^{4} \\
& +\left(3536+7566 z+7683 z^{2}+4442 z^{3}+1479 z^{4}+262 z^{5}+19 z^{6}\right) y^{5}+\cdots, \\
& \widehat{f}_{\square, \square, \square}=3 y^{2}-(36+7 z) y^{3}+\left(531+396 z+114 z^{2}+12 z^{3}\right) y^{4} \\
& -\left(8472+15210 z+13026 z^{2}+6399 z^{3}+1830 z^{4}+282 z^{5}+18 z^{6}\right) y^{5}+\cdots,
\end{aligned}
$$

and so on. For representations involving only one nontrivial representation, the degeneracies obtained above agree with the ones obtained in B-model computations [11] (see also [49]) and in A-model computations through localization 445,50.

One can also compute the amplitudes in nontrivial framings, just by framing the trivalent vertex in the appropriate way. For a single nontrivial representation with framing $n$, we find $N_{(\square, \cdot, \cdot), g, d}(n)=(-1)^{n} N_{(\square, \cdot, \cdot), g, d}(0)$, and

$$
\begin{aligned}
\widehat{f}_{\text {口, }, \cdot(n)=} & \frac{1}{8}\left(1-(-1)^{n}-2 n^{2}\right)+\frac{1}{96}\left(-3+3(-1)^{n}+8 n^{2}-2 n^{4}\right) z+\cdots \\
& +\left(n^{2}+\frac{1}{12} n^{2}\left(n^{2}-1\right) z+\cdots\right) y \\
& +\left(\frac{1}{4}\left(-1+(-1)^{n}-14 n^{2}\right)+\frac{1}{48}\left(3-3(-1)^{n}+8 n^{2}-14 n^{4}\right) z+\cdots\right) y^{2}+\cdots, \\
\widehat{f}_{\text {日, }, \cdot}(n)= & \frac{1}{8}\left(-1+(-1)^{n}-4 n-2 n^{2}\right)+\frac{1}{96}\left(3-3(-1)^{n}+8 n-4 n^{2}-8 n^{3}-2 n^{4}\right) z+\cdots \\
& +\left((n+1)^{2}+\frac{1}{12} n(1+n)^{2}(n+2) z+\cdots\right) y \\
& +\left(\frac{1}{4}\left(-15-(-1)^{n}-28 n-14 n^{2}\right)\right. \\
& \left.+\frac{1}{48}\left(-3+3(-1)^{n}-40 n-76 n^{2}-56 n^{3}-14 n^{4}\right) z+\cdots\right) y^{2}+\cdots,
\end{aligned}
$$

\subsection{Example $V$}




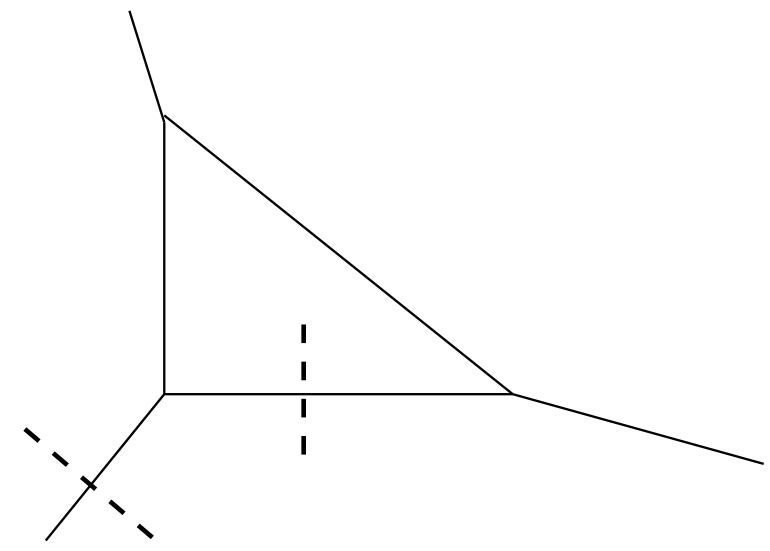

Fig. 15 The $\mathcal{O}(-3) \rightarrow \mathbf{P}^{\mathbf{2}}$ with an outer brane and another brane in an inner edge.

So far we have considered open string amplitudes where D-branes were sitting on outer edges, but our formalism also allows to compute amplitudes with branes in inner edges, as we saw in (3.16). A simple example of such a situation is local $\mathbf{P}^{\mathbf{2}}$ with an inner and an outer brane, as depicted in fig. 15. The framings are as in fig. 13. The prediction for this amplitude is

$$
\begin{array}{r}
Z\left(V_{1}, V_{2}\right)=\sum_{R_{i}, Q_{i}}(-1)^{s\left(R_{i}, Q_{i}\right)} q^{f\left(R_{i}, Q_{i}\right)} e^{-L\left(R_{i}, Q_{i}\right)} C_{Q_{1} R_{2} R_{3} \otimes Q_{2}} \\
C \cdot R_{3}^{t} \otimes Q_{3} R_{1}^{t} C_{\cdot R_{1} R_{2}^{t}} \operatorname{Tr}_{Q_{1}} V_{1} \operatorname{Tr}_{Q_{2}} V_{2} \operatorname{Tr}_{Q_{3}} V_{2}^{-1}
\end{array}
$$

where

$$
\begin{gathered}
L\left(R_{i}, Q_{i}\right)=\sum_{i} \ell\left(R_{i}\right) t+\ell\left(Q_{1}\right) r_{1}+\ell\left(Q_{2}\right) r_{2}+\ell\left(Q_{3}\right)\left(t-r_{2}\right) \\
f\left(R_{i}, Q_{i}\right)=\kappa_{R_{1}}+\kappa_{R_{2}}+n \kappa_{Q_{1}} / 2+p \kappa_{R_{3} \otimes Q_{2}} / 2+(p+2) \kappa_{R_{3}^{t} \otimes Q_{3}} / 2, \\
s\left(R_{i}, Q_{i}\right)=\sum_{i} \ell\left(R_{i}\right)+n \ell\left(Q_{1}\right)+p \ell\left(R_{3} \otimes Q_{2}\right)+(p+2) \ell\left(R_{3}^{t} \otimes Q_{3}\right)
\end{gathered}
$$

The integers $p$ and $n$ correspond to the framing of the inner brane and the outer branes, respectively. $p$ is related to the framing $p^{\prime}$ in the B-model of [1] by $p=-1-p^{\prime}$. We can again compute the generating functionals $\hat{f}$ for degeneracies of BPS states for different representations. We present some results corresponding to $p=-1$, and $n=0$ (for the 
inner brane, this is the zero framing of [1] ) where we absorb $e^{-r_{i}}$ in $V_{i}$ :

$$
\begin{aligned}
& \widehat{f}_{\cdot, \mathbf{\square}, \cdot}=-1+y-(5+z) y^{2}+\left(40+31 z+9 z^{2}+z^{3}\right) y^{3} \\
& -\left(399+743 z+648 z^{2}+322 z^{3}+94 z^{4}+15 z^{5}+z^{6}\right) y^{4} \\
& +\left(4524+16146 z+29256 z^{2}+33523 z^{3}+26079 z^{4}+14151 z^{5}+5364 z^{6}\right. \\
& \left.+1390 z^{7}+234 z^{8}+23 z^{9}+z^{10}\right) y^{5}+\cdots, \\
& \widehat{f}_{\cdot, \cdot, \square}=-1+2 y-(12+3 z) y+\left(104+96 z+33 z^{2}+4 z^{3}\right) y^{3} \\
& -\left(1085+2328 z+2334 z^{2}+1315 z^{3}+423 z^{4}+72 z^{5}+5 z^{6}\right) y^{4} \\
& -\left(12660+50874 z+103683 z^{2}+133002 z^{3}+114732 z^{4}+68040 z^{5}+27711 z^{6}\right. \\
& \left.+7590 z^{7}+1332 z^{8}+135 z^{9}+6 z^{10}\right) y^{5}+\cdots, \\
& \widehat{f}_{\square, \square, \cdot}=-1+y-(6+z) y^{2}+\left(59+39 z+10 z^{2}+z^{3}\right) y^{3} \\
& -\left(706+1152 z+895 z^{2}+403 z^{3}+108 z^{4}+16 z^{5}+z^{6}\right) y^{4} \\
& +\left(9372+29927 z+48964 z^{2}+51169 z^{3}+36663 z^{4}+18485 z^{5}+6561 z^{6}+1603 z^{7}\right. \\
& \left.+256 z^{8}+24 z^{9}+z^{10}\right) y^{5}+\cdots, \\
& \widehat{f}_{\cdot, \boldsymbol{\square}, \boldsymbol{\square}}=2 y^{2}-\left(46+30 z+5 z^{2}\right) y^{3}+\left(852+1682 z+1285 z^{2}+536 z^{3}+111 z^{4}+9 z^{5}\right) y^{4} \\
& -\left(14848+55104 z+101054 z^{2}+113629 z^{3}+83274 z^{4}+40375 z^{5}+12800 z^{6}\right. \\
& \left.+2544 z^{7}+287 z^{8}+14 z^{9}\right) y^{5}+\cdots,
\end{aligned}
$$

and so on. The results for $\widehat{f}_{\cdot, R}$, and $\widehat{f}_{\cdot, \cdot, R}$ correspond to inner branes with positive and negative winding numbers, respectively, and they agree with the B-model results of [11] in the case of disc amplitudes. For higher genus and/or number of holes, our results agree with those obtained through localization in [50]. The amplitudes with two nontrivial representations can be also obtained through localization, and in all cases we have found perfect agreement with the above results.

\subsection{Example VI}

We now consider more complicated examples of closed string amplitudes. Consider for example the toric diagram in fig. 16. There are three Kähler parameters involved, $s$, $t_{1}$ and $t_{2}$, as indicated in the figure. The amplitude is symmetric in $t_{1}, t_{2}$. When, say, $t_{2}$ is taken to infinity, the resulting geometry is that of a local Hirzebruch surface $\mathbf{F}_{1}$, where $s$ corresponds to the Kähler parameter of the base in local $\mathbf{F}_{1}$, while $t_{1}$ corresponds to the 


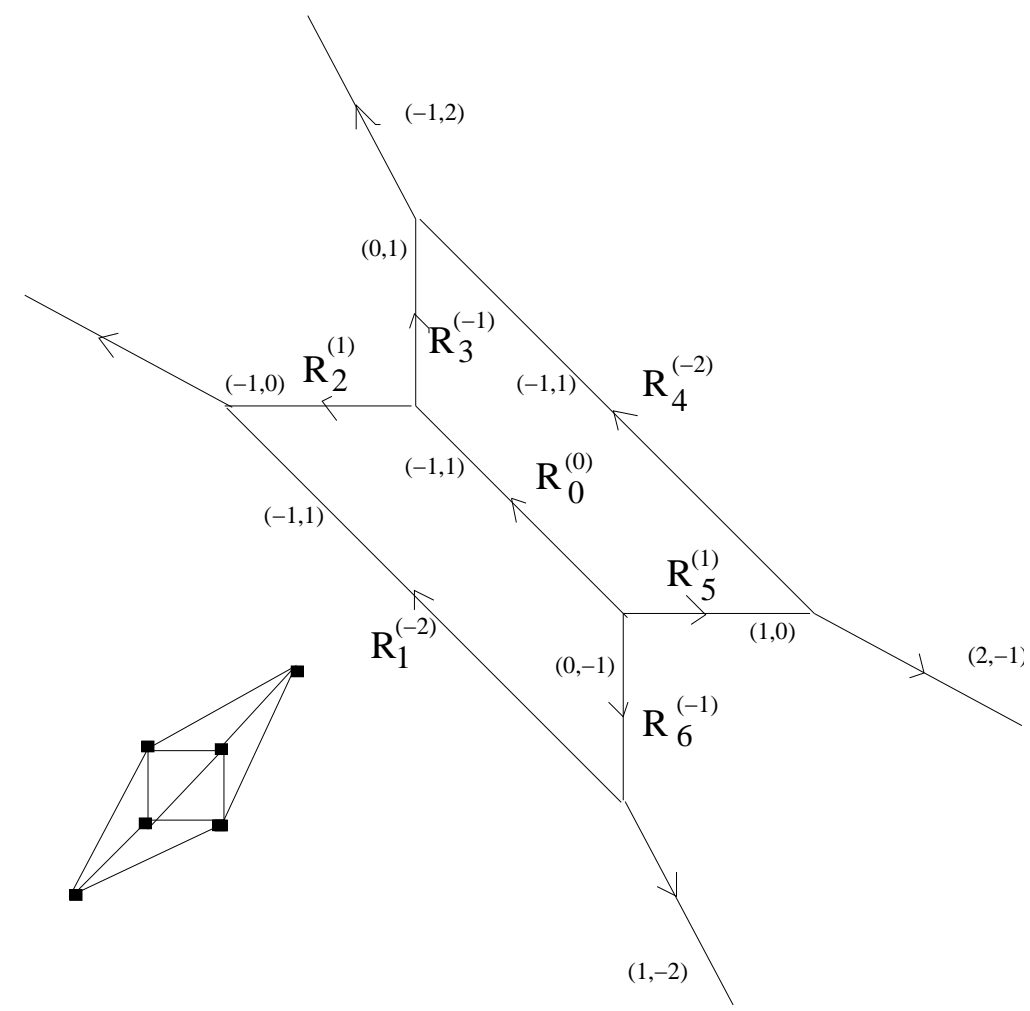

Fig. 16 There are three Kähler parameters in the problem. The size of leg corresponding to $R_{0}$ is $s$, and the sizes of $R_{3}$ and $R_{2}$ correspond to $t_{1}, t_{2}$, respectively.

fiber. The amplitude for fig. 16 can be computed by using our rules in section 3 , and the result is:

$$
\begin{gathered}
Z(X)=\sum_{R_{0} \ldots 6}(-1)^{\ell\left(R_{0}\right)+\ell\left(R_{1}\right)+\ell\left(R_{4}\right)} q^{\kappa_{R_{1}}} C_{R_{1}^{t} \cdot R_{2}^{t}} q^{-\kappa_{R_{2}} / 2} C_{R_{2} R_{3} R_{0}^{t}} q^{\kappa_{R_{3}} / 2} C_{R_{3}^{t} \cdot R_{4}^{t}} \\
q^{-\kappa_{R_{4}}} C_{R_{4} \cdot R_{5}^{t}} q^{-\kappa_{R_{5}} / 2} C_{R_{5} R_{6} R_{0}} q^{\kappa_{R_{6}} / 2} C_{R_{6}^{t} \cdot R_{1}} e^{-L\left(R_{i}\right)}
\end{gathered}
$$

where we wrote

$$
L\left(R_{i}\right)=\left(\ell\left(R_{0}\right)+\ell\left(R_{1}\right)+\ell\left(R_{4}\right)\right) s+\left(\ell\left(R_{2}\right)+\ell\left(R_{6}\right)\right) t_{1}+\left(\ell\left(R_{3}\right)+\ell\left(R_{5}\right)\right) t_{2} .
$$

Notice that when $t_{2} \rightarrow \infty$ (9.13) becomes the amplitude for local $\mathbf{F}_{1}$, which was computed from Chern-Simons theory in [5,6] with the techniques of [3]. We will write the answer in terms of a generating functional $\mathcal{F}_{g}$ of BPS degeneracies at genus $g$, as

$$
F_{g}\left(s, t_{1}, t_{2}\right)=\sum_{\ell=0}^{\infty} \mathrm{e}^{-\ell s} \mathcal{F}_{\ell}^{g}\left(t_{1}, t_{2}\right)
$$

where

$$
\mathcal{F}_{\ell}^{g}\left(t_{1}, t_{2}\right)=\sum_{d_{1}, d_{2}} n_{\ell, d_{1}, d_{2}}^{g} q_{1}^{d_{1}} q_{2}^{d_{2}}
$$


and we have written $q_{i}=\mathrm{e}^{-t_{i}}$ (these shouldn't be confused with the Chern-Simons variable introduced before). We then find, up to order four in $q_{i}$, the following results (symmetrization w.r.t. $q_{1}, q_{2}$ is understood):

$$
\begin{aligned}
& \mathcal{F}_{0}^{0}\left(t_{1}, t_{2}\right)=-2 q_{1}-2 q_{1} q_{2}+\cdots \\
& \mathcal{F}_{1}^{0}\left(t_{1}, t_{2}\right)=-1-3 q_{1}-5 q_{1}^{2}-7 q_{1}^{3}-9 q_{1}^{4}-4 q_{1} q_{2}-8 q_{1} q_{2}^{2}-12 q_{1} q_{2}^{3}-16 q_{1} q_{2}^{4}-9 q_{1}^{2} q_{2}^{2} \\
&-15 q_{1}^{2} q_{2}^{3}-21 q_{1}^{2} q_{2}^{4}-16 q_{1}^{3} q_{2}^{3}-24 q_{1}^{3} q_{2}^{4}-25 q_{1}^{4} q_{2}^{4}+\cdots, \\
& \mathcal{F}_{2}^{0}\left(t_{1}, t_{2}\right)=-6 q_{1}^{2}-32 q_{1}^{3}-110 q_{1}^{4}-10 q_{1} q_{2}^{2}-70 q_{1} q_{2}^{3}-270 q_{1} q_{2}^{4}-32 q_{1}^{2} q_{2}^{2}-126 q_{1}^{2} q_{2}^{3} \\
&-456 q_{1}^{2} q_{2}^{4}-300 q_{1}^{3} q_{2}^{3}-784 q_{1}^{3} q_{2}^{4}-1584 q_{1}^{4} q_{2}^{4}+\cdots, \\
& \mathcal{F}_{2}^{1}\left(t_{1}, t_{2}\right)= 9 q_{1}^{3}+68 q_{1}^{4}+16 q_{1} q_{2}^{3}+144 q_{1} q_{2}^{4}+21 q_{1}^{2} q_{2}^{3}+204 q_{1}^{2} q_{2}^{4}+59 q_{1}^{3} q_{2}^{3} \\
&+297 q_{1}^{3} q_{2}^{4}+684 q_{1}^{4} q_{2}^{4}+\cdots, \\
& \mathcal{F}_{2}^{2}\left(t_{1}, t_{2}\right)=-12 q_{1}^{4}-22 q_{1} q_{2}^{4}-30 q_{1}^{2} q_{2}^{4}-36 q_{1}^{3} q_{2}^{4}-94 q_{1}^{4} q_{2}^{4}+\cdots, \\
& \mathcal{F}_{3}^{0}\left(t_{1}, t_{2}\right)=27 q_{1}^{3}+286 q_{1}^{4}+64 q_{1} q_{2}^{3}+800 q_{1} q_{2}^{4}+25 q_{1}^{2} q_{2}^{2}+266 q_{1}^{2} q_{2}^{3}+1998 q_{1}^{2} q_{2}^{4} \\
&+1332 q_{1}^{3} q_{2}^{3}+6260 q_{1}^{3} q_{2}^{4}+21070 q_{1}^{4} q_{2}^{4}+\cdots, \\
& \mathcal{F}_{3}^{1}\left(t_{1}, t_{2}\right)=-10 q_{1}^{3}-288 q_{1}^{4}-18 q_{1} q_{2}^{3}-688 q_{1} q_{2}^{4} \\
& \quad-64 q_{1}^{2} q_{2}^{3}-1404 q_{1}^{2} q_{2}^{4}-516 q_{1}^{3} q_{2}^{3}-4372 q_{1}^{3} q_{2}^{4}-18498 q_{1}^{4} q_{2}^{4}+\cdots, \\
& \mathcal{F}_{3}^{2}\left(t_{1}, t_{2}\right)=108 q_{1}^{4}+224 q_{1} q_{2}^{4}+375 q_{1}^{2} q_{2}^{4}+49 q_{1}^{3} q_{2}^{3}+1168 q_{1}^{3} q_{2}^{4}+6837 q_{1}^{4} q_{2}^{4}+\cdots, \\
& \mathcal{F}_{3}^{3}\left(t_{1}, t_{2}\right)=-14 q_{1}^{4}-26 q_{1} q_{2}^{4}-36 q_{1}^{2} q_{2}^{4}-114 q_{1}^{3} q_{2}^{4}-1196 q_{1}^{4} q_{2}^{4}+\cdots, \\
& \mathcal{F}_{3}^{4}\left(t_{1}, t_{2}\right)=81 q_{1}^{4} q_{2}^{4}+\cdots,
\end{aligned}
$$

These numbers agree with the results obtained with localization 8 . Notice that, at genus 0 and for $t_{2} \rightarrow \infty$, the above results coincide with the results for local $\mathbf{F}_{1}$ presented for example in 43.

One can find a similar result for the $A_{2}$ fibration over $\mathbf{P}^{\mathbf{1}}$. In this case, the amplitude reads

$$
\begin{gathered}
Z(X)=\sum_{R_{0 \ldots 6}} q^{5 \kappa_{R_{1}} / 2} C_{R_{1}^{t} \cdot R_{2}^{t}} q^{-\kappa_{R_{2}} / 2} C_{R_{2} R_{3} R_{0}^{t}} q^{\kappa_{R_{3}} / 2+3 \kappa_{R_{0}} / 2} C_{R_{3}^{t} \cdot R_{4}^{t}} \\
q^{\kappa_{R_{4}} / 2} C_{R_{4} \cdot R_{5}^{t}} q^{-\kappa_{R_{5}} / 2} C_{R_{5} R_{6} R_{0}} q^{\kappa_{R_{6}} / 2} C_{R_{6}^{t} \cdot R_{1}} e^{-L\left(R_{i}\right)}
\end{gathered}
$$

where now

$$
\begin{aligned}
L\left(R_{i}\right) & =\left(\ell\left(R_{0}\right)+\ell\left(R_{1}\right)+\ell\left(R_{4}\right)\right) s \\
& +\left(4 \ell\left(R_{1}\right)+\ell\left(R_{2}\right)+\ell\left(R_{6}\right)\right) t_{1}+\left(2 \ell\left(R_{0}\right)+2 \ell\left(R_{1}\right)+\ell\left(R_{3}\right)+\ell\left(R_{5}\right)\right) t_{2} .
\end{aligned}
$$

8 In order to compare with the results using localization and mirror symmetry, we have redefined $g_{s} \rightarrow i g_{s}$ and therefore $n_{Q}^{g} \rightarrow(-1)^{g-1} n_{Q}^{g}$. 
Here, $s$ corresponds to the Kähler parameter of the base of the fibration, and $t_{1}, t_{2}$ correspond to the Kähler parameters of the fibers. Denoting the generating functional as before, we obtain in this case,

$$
\begin{aligned}
\mathcal{F}_{0}^{0}\left(t_{1}, t_{2}\right) & =-2 q_{1}-2 q_{2}-2 q_{1} q_{2}, \\
\mathcal{F}_{1}^{0}\left(t_{1}, t_{2}\right) & =-1-2 q_{2}-4 q_{2}^{2}-6 q_{2}^{3}-8 q_{2}^{4}-2 q_{1} q_{2}-6 q_{1} q_{2}^{2}-10 q_{1} q_{2}^{3}-14 q_{1} q_{2}^{4}-6 q_{1}^{2} q_{2}^{2} \\
& -12 q_{1}^{2} q_{2}^{3}-18 q_{1}^{2} q_{2}^{4}-4 q_{1}^{3} q_{2}^{2}-12 q_{1}^{3} q_{2}^{3}-20 q_{1}^{3} q_{2}^{4}-6 q_{1}^{4} q_{2}^{2}-10 q_{1}^{4} q_{2}^{3}-20 q_{1}^{4} q_{2}^{4}+\cdots, \\
\mathcal{F}_{2}^{0}\left(t_{1}, t_{2}\right) & =-6 q_{2}^{3}-32 q_{2}^{4}-10 q_{1} q_{2}^{3}-70 q_{1} q_{2}^{4}-12 q_{1}^{2} q_{2}^{3}-96 q_{1}^{2} q_{2}^{4}-12 q_{1}^{3} q_{2}^{3}-110 q_{1}^{3} q_{2}^{4} \\
& -10 q_{1}^{4} q_{2}^{3}-112 q_{1}^{4} q_{2}^{4}+\cdots, \\
\mathcal{F}_{2}^{1}\left(t_{1}, t_{2}\right) & =9 q_{1}^{4}+16 q_{1} q_{2}^{4}+21 q_{1}^{2} q_{2}^{4}+24 q_{1}^{3} q_{2}^{4}+25 q_{1}^{4} q_{2}^{4}+\cdots,
\end{aligned}
$$

and so on, again in agreement with the results for genus zero in [43]. We have also checked some of these results at higher genus with localization techniques.

\section{Acknowledgments}

We would like to thank D.-E.Diaconescu, R. Dijkgraaf, J. Gomis, A. Grassi, A. Iqbal, A. Kapustin, S. Katz, V. Kazakov, I. Kostov, C-C. Liu, H. Ooguri, J. Schwarz, S. Shenker and E. Zaslow for valuable discussions (and the cap!). The research of MA and CV was supported in part by NSF grants PHY-9802709 and DMS-0074329. In addition, CV thanks the hospitality of the theory group at Caltech, where he is a Gordon Moore Distinguished Scholar. M.A. is grateful to the Caltech theory group for hospitality during part of this work. A.K. is supported in part by the DFG grant KL-1070/2-1. 


\section{References}

[1] E. Witten, 'Topological sigma models," Commun. Math. Phys. 118, 411 (1988). "On the structure of the topological phase of two-dimensional gravity," Nucl. Phys. B 340, 281 (1990).

[2] R. Gopakumar and C. Vafa, "On the gauge theory/geometry correspondence," hepth/9811131, Adv. Theor. Math. Phys. 3 (1999) 1415.

[3] M. Aganagic, M. Mariño and C. Vafa, "All loop topological string amplitudes from Chern-Simons theory," hep-th/0206164.

[4] D. E. Diaconescu, B. Florea and A. Grassi, "Geometric transitions and open string instantons," hep-th/0205234. "Geometric transitions, del Pezzo surfaces and open string instantons," hep-th/0206163.

[5] A. Iqbal, "All genus topological string amplitudes and 5-brane webs as Feynman diagrams," hep-th/0207114; A. Iqbal and A.K. Kashani-Poor, to appear.

[6] A. Iqbal and A. K. Kashani-Poor, "Instanton counting and Chern-Simons theory," hep-th/0212279.

[7] D.-E. Diaconescu and A. Grassi, unpublished manuscript.

[8] M. Bershadsky, S. Cecotti, H. Ooguri and C. Vafa, "Kodaira-Spencer theory of gravity and exact results for quantum string amplitudes," hep-th/9309140, Commun. Math. Phys. 165 (1994) 311.

[9] E. Witten, "Phases of $\mathrm{N}=2$ theories in two dimensions," hep-th/9301042, Nucl. Phys. B 403, 159 (1993).

[10] P. S. Aspinwall, B. R. Greene and D. R. Morrison, "Calabi-Yau moduli space, mirror manifolds and spacetime topology change in string theory," Nucl. Phys. B 416, 414 (1994) arXiv:hep-th/9309097.

[11] M. Aganagic, A. Klemm and C. Vafa, "Disk instantons, mirror symmetry and the duality web," hep-th/0105045, Z. Naturforsch. A 57 (2002) 1.

[12] O. Aharony and A. Hanany, "Branes, superpotentials and superconformal fixed points," hep-th/9704170, Nucl. Phys. B 504 (1997) 239. O. Aharony, A. Hanany and B. Kol, "Webs of $(\mathrm{p}, \mathrm{q}) 5$-branes, five dimensional field theories and grid diagrams," hep-th/9710116, JHEP 9801 (1998) 002.

[13] N. C. Leung and C. Vafa, "Branes and toric geometry," hep-th/9711013, Adv. Theor. Math. Phys. 2 (1998) 91.

[14] K. Hori and C. Vafa, "Mirror symmetry," hep-th/0002222.

[15] K. Hori, A. Iqbal and C. Vafa, "D-branes and mirror symmetry," arXiv:hepth/0005247.

[16] M. Kontsevich, "Enumeration of rational curves via torus actions," hep-th/9405035, in The moduli space of curves, p. 335, Birkhäuser, 1995.

[17] R. Harvey and H.B. Lawson Jr., "Calibrated geometries," Acta Math. 148 (1982) 47. 
[18] E. Witten, "Quantum field theory and the Jones polynomial," Commun. Math. Phys. 121 (1989) 351.

[19] M. Aganagic and C. Vafa, "Mirror symmetry, D-branes and counting holomorphic discs," hep-th/0012041.

[20] M. Mariño and C. Vafa, "Framed knots at large $N$," hep-th/0108064.

[21] C. Vafa, "Brane/anti-brane systems and $U(N \mid M)$ supergroup," hep-th/0101218.

[22] H. Ooguri and C. Vafa, "Knot invariants and topological strings," hep-th/9912123, Nucl. Phys. B 577 (2000) 419.

[23] N. A. Nekrasov, "Seiberg-Witten prepotential from instanton counting," arXiv:hepth/0206161.

[24] A. S. Losev, A. Marshakov and N. A. Nekrasov, "Small instantons, little strings and free fermions," arXiv:hep-th/0302191.

[25] Work in progress with Robbert Dijkgraaf.

[26] N. Ishibashi, Y. Matsuo and H. Ooguri, "Soliton Equations And Free Fermions On Riemann Surfaces," Mod. Phys. Lett. A 2, 119 (1987).

[27] C. Vafa, "Operator Formulation On Riemann Surfaces," Phys. Lett. B 190, 47 (1987).

[28] L. Álvarez-Gaumé, C. Gómez, G. W. Moore and C. Vafa, "Strings In The Operator Formalism," Nucl. Phys. B 303, 455 (1988).

[29] M. Aganagic, A. Klemm, M. Mariño and C. Vafa, "Matrix model as a mirror of Chern-Simons theory," arXiv:hep-th/0211098.

[30] S. Shenker, private communication, 1995.

[31] H. Ooguri and C. Vafa, "Worldsheet derivation of a large $N$ duality," hep-th/0205297, Nucl. Phys. B 641, 3 (2002).

[32] E. Witten, "Chern-Simons gauge theory as a string theory," hep-th/9207094, in The Floer memorial volume, H. Hofer, C.H. Taubes, A. Weinstein and E. Zehner, eds., Birkhäuser 1995, p. 637.

[33] J.M.F. Labastida and M. Mariño, "Polynomial invariants for torus knots and topological strings," hep-th/0004196, Commun. Math. Phys. 217 (2001) 423.

[34] J.M.F. Labastida, M. Mariño and C. Vafa, "Knots, links and branes at large N," hep-th/0010102, JHEP 0011 (2000) 007.

[35] P. Ramadevi and T. Sarkar, "On link invariants and topological string amplitudes," Nucl. Phys. B 600, 487 (2001) arXiv:hep-th/0009188.

[36] E. Verlinde, "Fusion rules and modular transformations in 2-D conformal field theory," Nucl. Phys. B 300 (1988) 360.

[37] H.R. Morton and S.G. Lukac, "The HOMFLY polynomial of the decorated Hopf link," math.GT/0108011.

[38] S.G. Lukac, "HOMFLY skeins and the Hopf link," Ph.D. Thesis, June 2001, in http://www.liv.ac.uk su14/knotgroup.htm 
[39] I.G. Macdonald, Symmetric functions and Hall polynomials, 2nd edition, Oxford University Press, 1995.

[40] R. Gopakumar and C. Vafa, "M-theory and topological strings, II," hep-th/9812127.

[41] I. Antoniadis, E. Gava, K. S. Narain and T. R. Taylor, "Topological amplitudes in string theory," Nucl. Phys. B 413, 162 (1994) arXiv:hep-th/9307158.

[42] J.M.F. Labastida and M. Mariño, "A new point of view in the theory of knot and link invariants," math.QA/0104180, J. Knot Theory Ramifications 11 (2002) 173.

[43] T. M. Chiang, A. Klemm, S. T. Yau and E. Zaslow, "Local mirror symmetry: Calculations and interpretations," hep-th/9903053, Adv. Theor. Math. Phys. 3 (1999) 495.

[44] S. Hosono, "Counting BPS states via holomorphic anomaly equations," hep-th/0206206.

[45] T. Graber and E. Zaslow, "Open-string Gromov-Witten invariants: calculations and a mirror 'theorem'," hep-th/0109075.

[46] A. Klemm and E. Zaslow, "Local mirror symmetry at higher genus," hep-th/9906046, in Winter School on Mirror Symmetry, Vector bundles and Lagrangian Submanifolds, p. 183, American Mathematical Society 2001.

[47] S. Katz and C-C. Liu, "Enumerative geometry of stable maps with Lagrangian boundary conditions and multiple covers of the disc," math.AG/0103074, Adv. Theor. Math. Phys. 5 (2002) 1.

[48] C. Faber, "Algorithms for computing intersection numbers of curves, with an application to the class of the locus of Jacobians," alg-geom/9706006, in New trends in algebraic geometry, Cambridge Univ. Press, 1999.

[49] W. Lerche and P. Mayr, "On $\mathcal{N}=1$ mirror symmetry for open type II strings," hepth/0111113. S. Govindarajan, T. Jayaraman and T. Sarkar, "Disc instantons in linear sigma models," hep-th/0108234, Nucl. Phys. B 646, 498 (2002).

[50] P. Mayr, "Summing up open string instantons and $\mathcal{N}=1$ string amplitudes," hepth/0203237. 